\title{
LECTURES ON DUFLO ISOMORPHISMS IN LIE ALGEBRAS AND COMPLEX GEOMETRY
}

\author{
DAMIEN CALAQUE AND CARLO ROSSI
}

\begin{abstract}
For a complex manifold the Hochschild-Kostant-Rosenberg map does not respect the cup product on cohomology, but one can modify it using the square root of the Todd class in such a way that it does. This phenomenon is very similar to what happens in Lie theory with the Duflo-Kirillov modification of the Poincaré-Birkhoff-Witt isomorphism.

In these lecture notes (lectures were given by the first author at ETH-Zürich in fall 2007) we state and prove Duflo-Kirillov theorem and its complex geometric analogue. We take this opportunity to introduce standard mathematical notions and tools from a very down-to-earth viewpoint.
\end{abstract}

\section{Contents}

Introduction

2

1. Lie algebra cohomology and the Duflo isomorphism 4

2. Hochschild cohomology and spectral sequences 10

3. Dolbeault cohomology and the Kontsevich isomorphism 16

4. Superspaces and Hochschild cohomology 21

5. The Duflo-Kontsevich isomorphism for $Q$-spaces 26

6. Configuration spaces and integral weights 31

7. The map $\mathcal{U}_{Q}$ and its properties $\quad 37$

8. The map $\mathcal{H}_{Q}$ and the homotopy argument 43

9. The explicit form of $\mathcal{U}_{Q} \quad 49$

10. Fedosov resolutions 54

Appendix A. Deformation-theoretical intepretation of the Hochschild cohomology of a complex manifold

References
60

68 


\section{INTRODUCTION}

Since the fundamental results by Harish-Chandra and others one knows that the algebra of invariant polynomials on the dual of a Lie algebra of a particular type (solvable [12], simple [18] or nilpotent) is isomorphic to the center of the enveloping algebra. This fact was generalized to an arbitrary finite-dimensional real Lie algebra by M. Duflo in 1977 [13]. His proof is based on the Kirillov's orbits method that parametrizes infinitesimal characters of unitary irreducible representations of the corresponding Lie group in terms of co-adjoint orbits (see e.g. [21]). This isomorphism is called the Duflo isomorphism. It happens to be a composition of the well-known Poincaré-Birkhoff-Witt isomorphism (which is only an isomorphism on the level of vector spaces) with an automorphism of the space of invariant polynomials whose definition involves the power series $j(x):=\sinh (x / 2) /(x / 2)$.

In 1997 Kontsevich [22] proposed another proof, as a consequence of his construction of deformation quantization for general Poisson manifolds. Kontsevich's approach has the advantage to work also for Lie super-algebras and to extend the Duflo isomorphism to a graded algebra isomorphism on the whole cohomology.

The inverse power series $j(x)^{-1}=(x / 2) / \sinh (x / 2)$ also appears in Kontsevich's claim that the Hochschild cohomology of a complex manifold is isomorphic as an algebra to the cohomology ring of the polyvector fields on this manifold. We can summarize the analogy between the two situations into the following array:

$$
\begin{array}{rlr}
\frac{\text { Lie algebra }}{\text { symmetric algebra }} & & \text { Complex geometry } \\
\text { (sheaf of) algebra of holomorphic polyvector fields } \\
\text { universal enveloping algebra } & \text { (sheaf of) algebra of holomorphic polydifferential operators } \\
\text { taking invariants } & \text { taking holomorphic sections } \\
\text { Chevalley-Eilenberg cohomology } & \text { Dolbeault (or Čech) cohomology }
\end{array}
$$

This set of lecture notes provides a comprehensible proof of the Duflo isomorphism and its complex geometric analogue in a unified framework, and gives in particular a satisfying explanation for the reason why the series $j(x)$ and its inverse appear. The proof is strongly based on Kontsevich's original idea, but actually differs from it (the two approaches are related by a conjectural Koszul type duality recently pointed out in [30], this duality being itself a manifestation of Cattaneo-Felder constructions for the quantization of a Poisson manifold with two coisotropic submanifolds [8]).

Notice that the mentioned series also appears in the wheeling theorem by Bar-Natan, Le and Thurston [4] which shows that two spaces of graph homology are isomorphic as algebras (see also [23] for a completely combinatorial proof of the wheeling theorem, based on Alekseev and Meinrenken's proof $[1,2]$ of the Duflo isomorphism for quadratic Lie algebras). Furthermore this power series also shows up in various index theorems (e.g. Riemann-Roch theorems).

Throughout these notes we assume that $k$ is a field with $\operatorname{char}(k)=0$. Unless otherwise specified, algebras, modules, etc... are over $k$.

Each section consists (more or less) of a single lecture.

Acknowledgements. The authors thank the participants of the lectures for their interest and excitement. They are responsible for the very existence of these notes, as well as for improvement of their quality. The first author is grateful to G. Felder who offered him the opportunity to give this series of lectures. He also thanks M. Van den Bergh for his 
kind collaboration in [6] and many enlighting discussions about this fascinating subject. His research is fully supported by the European Union thanks to a Marie Curie Intra-European Fellowship (contract number MEIF-CT-2007-042212). 


\section{Lie Algebra COHOMOlogy AND the Duflo isomorphism}

Let $\mathfrak{g}$ be a finite dimensional Lie algebra over $k$. In this section we state the Duflo theorem and its cohomological extension. We take this opportunity to introduce standard notions of (co)homological algebra and define the cohomology theory associated to Lie algebras, which is called Chevalley-Eilenberg cohomology.

\subsection{The original Duflo isomorphism.}

The Poincaré-Birkhoff-Witt theorem.

Remember the Poincaré-Birkhoff-Witt (PBW) theorem: the symmetrization map

$$
\begin{aligned}
I_{P B W}: S(\mathfrak{g}) & \longrightarrow U(\mathfrak{g}) \\
x^{n} & \longmapsto x^{n}(x \in \mathfrak{g}, n \in \mathbb{N})
\end{aligned}
$$

is an isomorphism of filtered vector spaces. Moreover it induces an isomorphism of graded algebras $S(\mathfrak{g}) \rightarrow \operatorname{Gr}(U(\mathfrak{g}))$.

This is well-defined since the $x^{n}(x \in \mathfrak{g})$ generate $S(\mathfrak{g})$ as a vector space. On monomials it gives

$$
I_{P B W}\left(x_{1} \cdots x_{n}\right)=\frac{1}{n !} \sum_{\sigma \in \mathfrak{S}_{n}} x_{\sigma_{1}} \cdots x_{\sigma_{n}} .
$$

Let us write $*$ for the associative product on $S(\mathfrak{g})$ defined as the pullback of the multiplication on $U(\mathfrak{g})$ through $I_{P B W}$. For any two homogeneous elements $u, v \in S(\mathfrak{g}), u * v=u v+$ l.o.t. (where l.o.t. stands for lower order terms).

$I_{P B W}$ is obviously NOT an algebra isomorphism unless $\mathfrak{g}$ is abelian (since $S(\mathfrak{g})$ is commutative while $U(\mathfrak{g})$ is not).

Geometric meaning of the PBW theorem.

Denote by $G$ the germ of $k$-analytic Lie group having $\mathfrak{g}$ as a Lie algebra.

Then $S(\mathfrak{g})$ can be viewed as the algebra of distributions on $\mathfrak{g}$ supported at the origin 0 with (commutative) product given by the convolution with respect to the (abelian) group law + on $\mathfrak{g}$.

In the same way $U(\mathfrak{g})$ can be viewed as the algebra of distributions on $G$ supported at the origin $e$ with product given by the convolution with respect to the group law on $G$.

One sees that $I_{P B W}$ is nothing but the transport of distributions through the exponential map exp : $\mathfrak{g} \rightarrow G$ (recall that it is a local diffeomorphism). The exponential map is obviously $A d$-equivariant. In the next paragraph we will translate this equivariance in algebraic terms.

$\mathfrak{g}$-module structure on $S(\mathfrak{g})$ and $U(\mathfrak{g})$.

On the one hand there is a $\mathfrak{g}$-action on $S(\mathfrak{g})$ obtained from the adjoint action $a d$ of $\mathfrak{g}$ on itself, extended to $S(\mathfrak{g})$ by derivations : for any $x, y \in \mathfrak{g}$ and $n \in \mathbb{N}^{*}$,

$$
\operatorname{ad}_{x}\left(y^{n}\right)=n[x, y] y^{n-1} .
$$

On the other hand there is also an adjoint action of $\mathfrak{g}$ on $U(\mathfrak{g})$ : for any $x \in \mathfrak{g}$ and $u \in U(\mathfrak{g})$,

$$
\operatorname{ad}_{x}(u)=x u-u x .
$$

It is an easy exercise to verify that $\operatorname{ad}_{x} \circ I_{P B W}=I_{P B W} \circ \operatorname{ad}_{x}$ for any $x \in \mathfrak{g}$.

Therefore $I_{P B W}$ restricts to an isomorphism (of vector spaces) from $S(\mathfrak{g})^{\mathfrak{g}}$ to the center $\mathcal{Z}(U \mathfrak{g})=U(\mathfrak{g})^{\mathfrak{g}}$ of $U \mathfrak{g}$.

Now we have commutative algebras on both sides. Nevertheless, $I_{P B W}$ is not yet an algebra isomorphism. Theorem 1.2 below is concerned with the failure of this map to respect the product. 
Duflo element $J$.

We define an element $J \in \widehat{S}\left(\mathfrak{g}^{*}\right)$ as follows:

$$
J:=\operatorname{det}\left(\frac{1-e^{-\mathrm{ad}}}{\mathrm{ad}}\right) .
$$

It can be expressed as a formal combination of the $c_{k}:=\operatorname{tr}\left((\mathrm{ad})^{k}\right)$.

Let us explain what this means. Recall that ad is the linear map $\mathfrak{g} \rightarrow \operatorname{End}(\mathfrak{g})$ defined by $\operatorname{ad}_{x}(y)=[x, y](x, y \in \mathfrak{g})$. Therefore ad $\in \mathfrak{g}^{*} \otimes \operatorname{End}(\mathfrak{g})$ and thus $(\operatorname{ad})^{k} \in T^{k}\left(\mathfrak{g}^{*}\right) \otimes \operatorname{End}(\mathfrak{g})$. Consequently $\operatorname{tr}\left((\mathrm{ad})^{k}\right) \in T^{k}\left(\mathfrak{g}^{*}\right)$ and we regard it as an elements of $S^{k}\left(\mathfrak{g}^{*}\right)$ through the projection $T\left(\mathfrak{g}^{*}\right) \rightarrow S\left(\mathfrak{g}^{*}\right)$.

Claim 1.1. $c_{k}$ is $\mathfrak{g}$-invariant.

Here the $\mathfrak{g}$-module structure on $S\left(\mathfrak{g}^{*}\right)$ is the coadjoint action on $\mathfrak{g}^{*}$ extended by derivations.

Proof. Let $x, y \in \mathfrak{g}$. Then

$$
\begin{aligned}
\left\langle y \cdot c_{k}, x^{n}\right\rangle & =-\left\langle c_{k}, \sum_{i=1}^{n} x^{i}[y, x] x^{n-i-1}\right\rangle=-\sum_{i=1}^{n} \operatorname{tr}\left(\operatorname{ad}_{x}^{i} \operatorname{ad}_{[y, x]} \operatorname{ad}_{x}^{n-i-1}\right) \\
& =-\sum_{i=1}^{n} \operatorname{tr}\left(\operatorname{ad}_{x}^{i}\left[\operatorname{ad}_{y}, \operatorname{ad}_{x}\right] \operatorname{ad}_{x}^{n-i-1}\right)=-\operatorname{tr}\left(\left[\operatorname{ad}_{y}, \operatorname{ad}_{x}^{n}\right]\right)=0
\end{aligned}
$$

This proves the claim.

\section{The Duflo isomorphism.}

Observe that an element $\xi \in \mathfrak{g}^{*}$ acts on $S(\mathfrak{g})$ as a derivation as follows: for any $x \in \mathfrak{g}$

$$
\xi \cdot x^{n}=n \xi(x) x^{n-1} .
$$

By extension an element $(\xi)^{k} \in S^{k}\left(\mathfrak{g}^{*}\right)$ acts as follows:

$$
(\xi)^{k} \cdot x^{n}=n \cdots(n-k+1) \xi(x)^{k} x^{n-k} .
$$

This way the algebra $\widehat{S}\left(\mathfrak{g}^{*}\right)$ acts on $S(\mathfrak{g}) .{ }^{1}$ Moreover, one sees without difficulty that $\widehat{S}\left(\mathfrak{g}^{*}\right)^{\mathfrak{g}}$ acts on $S(\mathfrak{g})^{\mathfrak{g}}$. We have:

Theorem 1.2 (Duflo,[13]). $I_{P B W} \circ J^{1 / 2}$. defines an isomorphism of algebras $S(\mathfrak{g})^{\mathfrak{g}} \rightarrow U(\mathfrak{g})^{\mathfrak{g}}$.

The proof we will give in these lectures is based on deformation theory and (co)homological algebra, following the deep insight of M. Kontsevich [22] (see also [29]).

Remark 1.3. $\quad c_{1}$ is a derivation of $S(\mathfrak{g})$ therefore $\exp \left(c_{1}\right)$ defines an algebra automorphism of $S(\mathfrak{g})$. Therefore one can obviously replace $J$ by the modified Duflo element

\subsection{Cohomology.}

$$
\widetilde{J}=\operatorname{det}\left(\frac{e^{\mathrm{ad} / 2}-e^{-\mathrm{ad} / 2}}{\mathrm{ad}}\right) \text {. }
$$

Our aim is to show that Theorem 1.2 is the degree zero part of a more general statement. For this we need a few definitions.

Definition 1.4. 1. A $D G$ vector space is a $\mathbb{Z}$-graded vector space $C^{\bullet}=\oplus_{n \in \mathbb{Z}} C^{n}$ equipped with a graded linear endomorphism $d: C \rightarrow C$ of degree one (i.e. $d\left(C^{n}\right) \subset C^{n+1}$ ) such that $d \circ d=0 . d$ is called the differential.

2. A DG (associative) algebra is a DG vector space $\left(A^{\bullet}, d\right)$ equipped with an associative product which is graded (i.e. $A^{k} \cdot A^{l} \subset A^{k+l}$ ) and such that $d$ is a degree one superderivation: for homogeneous elements $a, b \in A d(a \cdot b)=d(a) \cdot b+(-1)^{|a|} a \cdot d(b)$.

\footnotetext{
${ }^{1}$ This action can be regarded as the action of the algebra of differential operators with constant coefficients on $\mathfrak{g}^{*}$ (of possibly infinite degree) onto functions on $\mathfrak{g}^{*}$.
} 
3. A Let $\left(A^{\bullet}, d\right)$ be a DG algebra. A $D G A$-module is a DG vector space $\left(M^{\bullet}, d\right)$ equipped with an $A$-module structure which is graded (i.e. $A^{k} \cdot M^{l} \subset M^{m+l}$ ) and such that $d$ satisfies $d(a \cdot m)=d(a) \cdot m+(-1)^{|a|} a \cdot d(m)$ for homogeneous elements $a \in A, m \in M$.

4. A morphism of DG vector spaces (resp. DG algebras, DG $A$-modules) is a degree preserving linear map that intertwines the differentials (resp. and the products, the module structures).

DG vector spaces are also called cochain complexes (or simply complexes) and differentials are also known as coboundary operators. Recall that the cohomology of a cochain complex $\left(C^{\bullet}, d\right)$ is the graded vector space $H^{\bullet}(C, d)$ defined by the quotient $\operatorname{ker}(d) / \operatorname{im}(d)$ :

$$
H^{n}(C, d):=\frac{\left\{c \in C^{n} \mid d(c)=0\right\}}{\left\{b=d(a) \mid a \in C^{n-1}\right\}}=\frac{\{n \text {-cocycles }\}}{\{n \text {-coboundaries }\}} .
$$

Any morphism of cochain complexes induces a degree preserving linear map on the level of cohomology. The cohomology of a DG algebra is a graded algebra.

Example 1.5 (Differential-geometric induced DG algebraic structures). Let $M$ be a differentiable manifold. Then the graded algebra of differential forms $\Omega^{\bullet}(M)$ equipped with the de Rham differential $\mathrm{d}=d_{d R}$ is a DG algebra. Recall that for any $\omega \in \Omega^{n}(M)$ and $v_{0}, \ldots, v_{n} \in \mathfrak{X}(M)$

$$
\begin{aligned}
& \mathrm{d}(\omega)\left(u_{0}, \cdots, u_{n}\right):=\sum_{i=0}^{n}(-1)^{i} u_{i}\left(\omega\left(u_{0}, \ldots, \widehat{u_{i}}, \ldots, u_{n}\right)\right) \\
& +\sum_{0 \leq i<j \leq n}(-1)^{i+j} \omega\left(\left[u_{i}, u_{j}\right], u_{0}, \ldots, \widehat{u_{i}}, \ldots, \widehat{u_{j}}, \ldots, u_{n}\right) .
\end{aligned}
$$

In local coordinates $\left(x^{1}, \ldots, x^{n}\right)$, the de Rham differential reads $\mathrm{d}=\mathrm{d} x^{i} \frac{\partial}{\partial x^{i}}$. The corresponding cohomology is denoted by $H_{d R}^{\bullet}(M)$.

For any $C^{\infty}$ map $f: M \rightarrow N$ one has a morphism of DG algebras given by the pullback of forms $f^{*}: \Omega^{\bullet}(N) \rightarrow \Omega^{\bullet}(M)$.

Let $E \rightarrow M$ be a vector bundle and recall that a connection $\nabla$ on $M$ with values in $E$ is given by the data of a linear map $\nabla: \Gamma(M, E) \rightarrow \Omega(M, E)$ such that for any $f \in C^{\infty}(M)$ and $s \in \Gamma(M, E)$ one has $\nabla(f s)=d(f) s+f \nabla(s)$. Observe that it extends in a unique way to a degree one linear map $\nabla: \Omega^{\bullet}(M, E) \rightarrow \Omega^{\bullet}(M, E)$ such that for any $\xi \in \Omega^{\bullet}(M)$ and $s \in \Omega^{\bullet}(M, E), \nabla(\xi s)=\mathrm{d}(\xi) s+(-1)^{|\xi|} \xi \nabla(s)$. Therefore if the connection is flat (which is basically equivalent to the requirement that $\nabla \circ \nabla=0)$ then $\Omega^{\bullet}(M, E)$ becomes a DG $\Omega(M)$-module. Conversely, any differential $\nabla$ that turns $\Omega(M, E)$ in a DG $\Omega(M)$-module defines a flat connection.

Definition 1.6. A quasi-isomorphism is a morphism that induces an isomorphism on the level of cohomology.

Example 1.7 (Poincaré lemma). Let us regard $\mathbb{R}$ as a DG algebra concentrated in degree zero and with $d=0$. The inclusion $i:(\mathbb{R}, 0) \hookrightarrow\left(\Omega^{\bullet}\left(\mathbb{R}^{n}\right), \mathrm{d}\right)$ is a quasi-isomorphism of DG algebras. The proof of this claim is quite instructive as it makes use of a standard method in homological algebra:

Proof. Let us construct a degree -1 graded linear map $\kappa: \Omega^{\bullet}\left(\mathbb{R}^{n}\right) \rightarrow \Omega^{\bullet-1}\left(\mathbb{R}^{n}\right)$ such that

$$
\mathrm{d} \circ \kappa+\kappa \circ \mathrm{d}=\mathrm{id}-i \circ p,
$$

where $p: \Omega^{\bullet}(M) \rightarrow k$ takes the degree zero part of a form and evaluates it at the origin: $p(f(\underline{x}, \underline{\mathrm{d}} \underline{x}))=f(0,0)$ (here we write locally a form as a "function" of the "variables" $\left.x^{1}, \ldots, x^{n}, \mathrm{~d} x^{1}, \ldots, \mathrm{d} x^{n}\right)^{2}$. Then it is obvious that any closed form lies in the image of $i$ up

\footnotetext{
${ }^{2}$ This comment will receive a precise explanation in Section 4, where we consider superspaces.
} 
to an exact one. This is an exercise to check that $\kappa$ defined by $\kappa(1)=0$ and

$$
\kappa_{\mid \operatorname{ker}(p)}(f(\underline{x}, \mathrm{~d} \underline{x}))=x^{i} \iota_{\partial_{i}}\left(\int_{0}^{1} f(t \underline{x}, t \mathrm{~d} \underline{x}) \frac{d t}{t}\right)
$$

satisfies those conditions.

Notice that we have proved at the same time that $p:\left(\Omega^{\bullet}(M), \mathrm{d}\right) \rightarrow(k, 0)$ is also a quasiisomorphism. Moreover, one can check that $\kappa \circ \kappa=0$. This allows us to decompose $\Omega^{\bullet}(M)$ as $\operatorname{ker}(\Delta) \oplus \operatorname{im}(\mathrm{d}) \oplus \operatorname{im}(\kappa)$, where $\Delta$ is defined to be the l.h.s. of (1.1). $\Delta$ is often called the Laplacian and thus elements lying in its kernel are said harmonic ${ }^{3}$.

A historical remark.

Homological algebra is a powerful tool that was originally introduced in order to produce topological invariants. E.g. the de Rham cohomology: two homeomorphic differentiable manifolds have isomorphic de Rham cohomology.

The ideas involved in homological algebra probably goes back to the study of polyhedra: if we call $F$ the number of faces of a polyhedron, $E$ its numbers of edges and $V$ its number of vertices, then $F-E+V$ is a topological invariant. In particular if the polyhedron is homeomorphic to a sphere it equals 2 .

The name cohomology suggests that it comes with homology. Let us briefly say that homology deals with chain complexes: they are like cochain complexes but the differential has degree -1 . It is called the boundary operator and its name has a direct topological inspiration (e.g. the boundary of a face is a formal sum of edges).

\subsection{Chevalley-Eilenberg cohomology.}

The Chevalley-Eilenberg complex.

Let $V$ be a $\mathfrak{g}$-module. The associated Chevalley-Eilenberg complex $C^{\bullet}(\mathfrak{g}, V)$ is defined as follows: $C^{n}(\mathfrak{g}, V)=\wedge^{n}(\mathfrak{g})^{*} \otimes V$ is the space of linear maps $\wedge^{n}(\mathfrak{g}) \rightarrow V$ and the differential $d_{C}$ is defined on homogeneous elements by

$$
\begin{array}{r}
d_{C}(l)\left(x_{0}, \ldots, x_{n}\right):=\sum_{0 \leq i<j \leq n}(-1)^{i+j} l\left(\left[x_{i}, x_{j}\right], x_{0}, \ldots, \widehat{x_{i}}, \ldots, \widehat{x_{j}}, \ldots, x_{n}\right) \\
+\sum_{i=0}^{n}(-1)^{i} x_{i} \cdot l\left(x_{0}, \ldots, \widehat{x_{i}}, \ldots, x_{n}\right) .
\end{array}
$$

We prove below that $d_{C} \circ d_{C}=0$.

The corresponding cohomology is denoted $H^{\bullet}(\mathfrak{g}, V)$.

Remark 1.8. Below we implicitely identify $\wedge(\mathfrak{g})$ with antisymmetric elements in $T(\mathfrak{g})$. Namely, we define the total antisymmetrization operator alt $: T(\mathfrak{g}) \rightarrow T(\mathfrak{g})$ :

$$
\operatorname{alt}\left(x_{1} \otimes \cdots \otimes x_{n}\right):=\frac{1}{n !} \sum_{\sigma \in \mathfrak{S}_{n}}(-1)^{\sigma} x_{\sigma(1)} \otimes \cdots \otimes x_{\sigma(n)} .
$$

It is a projector, and it factorizes through an isomorphism $\wedge(\mathfrak{g}) \stackrel{\sim}{\longrightarrow} \operatorname{ker}($ alt - id $)$, that we also denote by alt. In particular this allows us to identify $\wedge\left(\mathfrak{g}^{*}\right)$ with $\wedge(\mathfrak{g})^{*}$.

\footnotetext{
${ }^{3}$ This terminology is chosen by analogy with the Hodge-de Rham decomposition of $\Omega^{\bullet}(M)$ when $M$ is a Riemannian manifold. Namely, let $*$ be the Hodge star operator and define $\kappa:= \pm * \mathrm{~d} *$. Then $\Delta$ is precisely the usual Laplacian, and harmonic forms provide representatives of de Rham cohomology classes.
} 
Cup product.

If $V=A$ is equipped with an associative $\mathfrak{g}$-invariant product, meaning that for any $x \in \mathfrak{g}$ and any $a, b \in A$

$$
x \cdot(a b)=(x \cdot a) b+a(x \cdot b),
$$

then $C^{\bullet}(\mathfrak{g}, A)$ naturally becomes a graded algebra with product $\cup$ defined as follows: for any $\xi, \eta \in \wedge\left(\mathfrak{g}^{*}\right)$ and $a, b \in A$

$$
(\xi \otimes a) \cup(\eta \otimes b)=\xi \wedge \eta \otimes a b .
$$

Another way to write the product is as follows: for $l: \wedge^{m}(\mathfrak{g})^{*} \rightarrow A, l^{\prime}: \wedge^{n}(\mathfrak{g})^{*} \rightarrow A$ and $x_{1}, \ldots, x_{m+n} \in \mathfrak{g}$

$$
\left(l \cup l^{\prime}\right)\left(x_{1}, \ldots, x_{m+n}\right)=\frac{1}{(m+n) !} \sum_{\sigma \in \mathfrak{S}_{m+n}}(-1)^{\sigma} l\left(x_{\sigma(1)}, \ldots, x_{\sigma(m)}\right) l^{\prime}\left(x_{\sigma(m+1)}, \ldots, x_{\sigma(m+n)}\right)
$$

Remark 1.9. Observe that since $l$ and $l^{\prime}$ are already antisymmetric then it is sufficient to take $\frac{m ! n !}{(m+n) !}$ times the sum over $(m, n)$-shuffles (i.e. $\sigma \in \mathfrak{S}_{m+n}$ such that $\sigma(1)<\cdots<\sigma(m)$ and $\sigma(m+1)<\cdots<\sigma(m+n)$.

Exercise 1.10. Check that $\cup$ is associative and satisfies

$$
d_{C}\left(l \cup l^{\prime}\right)=d_{C}(l) \cup l^{\prime}+(-1)^{|l|} l \cup d_{C}\left(l^{\prime}\right) .
$$

The Chevalley-Eilenberg complex is a complex.

In this paragraph we prove that $d_{C} \circ d_{C}=0$.

Let us first prove it in the case when $V=k$ is the trivial module. Let $\xi \in \mathfrak{g}^{*}$ and $x, y, z \in \mathfrak{g}$, then

$$
\begin{aligned}
d_{C} \circ d_{C}(\xi)(x, y, z) & =-d_{C}(\xi)([x, y], z)+d_{C}(\xi)([x, z], y)-d_{C}(\xi)([y, z], x) \\
& =\xi([[x, y], z]-[[x, z], y]+[[y, z], x])=0
\end{aligned}
$$

Since $\wedge\left(\mathfrak{g}^{*}\right)$ is generated as an algebra (with product $\cup=\wedge$ ) by $\mathfrak{g}^{*}$ then it follows from (1.2) that $d_{C} \circ d_{C}=0$.

Let us come back to the general case. Observe that $C^{\bullet}(\mathfrak{g}, V)=\wedge^{\bullet}\left(\mathfrak{g}^{*}\right) \otimes V$ is a graded $\wedge^{\bullet}\left(\mathfrak{g}^{*}\right)$-module: for any $\xi \in \wedge^{\bullet}\left(\mathfrak{g}^{*}\right)$ and $\eta \otimes v \in \wedge^{\bullet}\left(\mathfrak{g}^{*}\right) \otimes V$,

$$
\xi \cdot(\eta \otimes v):=(\xi \wedge \eta) \otimes v .
$$

Since $C^{\bullet}(\mathfrak{g}, V)$ is generated by $V$ as a graded $\wedge^{\bullet}\left(\mathfrak{g}^{*}\right)$-module, and thanks to the fact (the verification is left as an exercise) that

$$
d_{C}(\xi \cdot(\eta \otimes v))=d_{C}(\xi) \cdot(\eta \otimes v)+(-1)^{|\xi|} \xi \cdot d_{C}(\eta \otimes v),
$$

then it is sufficient to prove that $d_{C} \circ d_{C}(v)=0$ for any $v \in V$. We do this now: if $x, y \in \mathfrak{g}$ then

$$
\begin{aligned}
d_{C} \circ d_{C}(v)(x, y) & =-d_{C}(v)([x, y])+x \cdot d_{C}(v)(y)-y \cdot d_{C}(v)(x) \\
& =-[x, y] \cdot v+x \cdot(y \cdot v))-y \cdot(x \cdot v)=0 .
\end{aligned}
$$

Interpretation of $H^{0}(\mathfrak{g}, V), H^{1}(\mathfrak{g}, V)$ and $H^{2}(\mathfrak{g}, V)$.

We will now interpret the low degree components of Chevalley-Eilenberg cohomology.

- Obviously, the 0-th cohomology space $H^{0}(\mathfrak{g}, V)$ is equal to the space $V^{\mathfrak{g}}$ of $\mathfrak{g}$-invariant elements in $V$ (i.e. those elements on which the action is zero).

- 1-cocycles are linear maps $l: \mathfrak{g} \rightarrow V$ such that $l([x, y])=x \cdot l(y)-y \cdot l(x) b$ for $x, y \in \mathfrak{g}$. In other words 1-cocycles are $\mathfrak{g}$-derivations with values in $V$. 1-coboundaries are those derivations $l_{v}(v \in V)$ of the form $l_{v}(x)=x \cdot v(x \in \mathfrak{g})$, which are called inner derivations. Thus $H^{1}(\mathfrak{g}, V)$ is the quotient of the space of derivations by inner derivations.

- 2-cocycles are linear maps $\omega: \wedge^{2} \mathfrak{g} \rightarrow V$ such that

$$
\omega([x, y], z)+\omega([z, x], y)+\omega([y, z], x)-x \cdot \omega(y, z)+y \cdot \omega(x, z)-z \cdot \omega(y, z)=0 \quad(x, y, x \in \mathfrak{g}) .
$$


This last condition is equivalent to the requirement that the space $\mathfrak{g} \oplus V$ equipped with the bracket

$$
[x+u, y+v]=([x, y]+x \cdot v-y \cdot u)+\omega(x, y) \quad(x, y \in \mathfrak{g}, v, w \in V)
$$

is a Lie algebra. Such objects are called extensions of $\mathfrak{g}$ by $V$. 2-coboundaries $\omega=d_{C}(l)$ correspond exactly to those extensions that are trivial (i.e. such that the resulting Lie algebra structure on $\mathfrak{g} \oplus V$ is isomorphic to the one given by $\omega_{0}=0$; the isomorphism is given by $x+v \mapsto x+l(x)+v)$.

\subsection{The cohomological Duflo isomorphism.}

From the PBW isomorphism $I_{P B W}: S(\mathfrak{g}) \stackrel{\sim}{\longrightarrow} U(\mathfrak{g})$ of $\mathfrak{g}$-modules one obtains an isomorphism of cochain complexes $C^{\bullet}(\mathfrak{g}, S(\mathfrak{g})) \stackrel{\sim}{\longrightarrow} C^{\bullet}(\mathfrak{g}, U(\mathfrak{g}))$. This is obviously not a DG algebra morphism (even on the level of cohomology).

The following result is an extension of the Duflo Theorem 1.2. It has been rigourously proved by M. Pevzner and C. Torossian in [27], after the deep insight of M. Kontsevich.

Theorem 1.11. $I_{P B W} \circ J^{1 / 2}$. induces an isomorphism of algebras on the level of cohomology

$$
H^{\bullet}(\mathfrak{g}, S(\mathfrak{g})) \stackrel{\sim}{\longrightarrow} H^{\bullet}(\mathfrak{g}, U(\mathfrak{g})) .
$$

Again, one can obviously replace $J$ by $\widetilde{J}$. 


\section{HochSCHILD COHOMOLOGY AND SPECTRAL SEQUENCES}

In this section we define a cohomology theory for associative algebras, which is called Hochschild cohomology, and explain the meaning of it. We also introduce the notion of a spectral sequence and use it to prove that, for a Lie algebra $\mathfrak{g}$, the Hochschild cohomology of $U(\mathfrak{g})$ is the same as the Chevalley-Eilenberg cohomology of $\mathfrak{g}$.

\subsection{Hochschild cohomology.}

The Hochschild complex.

Let $A$ be an associative algebra and $M$ an $A$-bimodule (i.e. a vector space equipped with two commuting $A$-actions, one on the left and the other on the right).

The associated Hochschild complex $C^{\bullet}(A, M)$ is defined as follows: $C^{n}(A, M)$ is the space of linear maps $A^{\otimes n} \rightarrow M$ and the differential $d_{H}$ is defined on homogeneous elements by the formula

$$
\begin{array}{r}
d_{H}(f)\left(a_{0}, \ldots, a_{m}\right)=a_{0} f\left(a_{1}, \ldots, a_{m}\right)+\sum_{i=1}^{m}(-1)^{i} f\left(a_{0}, \ldots, a_{i-1} a_{i}, \ldots, a_{m}\right) \\
+(-1)^{m+1} f\left(a_{0}, \ldots, a_{m-1}\right) a_{m} .
\end{array}
$$

It is easy to prove that $d_{H} \circ d_{H}=0$.

The corresponding cohomology is denoted $H^{\bullet}(A, M)$.

If $M=B$ is an algebra such that for any $a \in A$ and any $b, b^{\prime} \in B a\left(b b^{\prime}\right)=(a b) b^{\prime}$ and $\left(b b^{\prime}\right) a=b\left(b^{\prime} a\right)$ (e.g. $B=A$ the algebra itself) then $\left(C^{\bullet}(A, B), d_{H}\right)$ becomes a DG algebra; the product $\cup$ is defined on homogeneous elements by

$$
f \cup g\left(a_{1}, \ldots, a_{m+n}\right)=f\left(a_{1}, \ldots, a_{m}\right) g\left(a_{m+1}, \ldots, a_{m+n}\right) .
$$

If $M=A$ then we write $H H^{\bullet}(A):=H^{\bullet}(A, A)$.

Interpretation of $H^{0}(A, M)$ and $H^{1}(A, M)$.

We will now interpret the low degree components of Hochschild cohomology.

- Obviously, the 0 -th cohomology space $H^{0}(A, M)$ is equal to the space $M^{A}$ of $A$-invariant elements in $M$ (i.e. those elements on which the left and right actions coincide). In the case $M=A$ is the algebra itself we then have $H^{0}(A, A)=\mathcal{Z}(A)$.

- 1-cocycles are linear maps $l: A \rightarrow M$ such that $l(a b)=a l(b)+l(a) b$ for $a, b \in A$, i.e. 1-cocycles are $A$-derivations with values in $M$. 1-coboundaries are those derivations $l_{m}$ $(m \in M)$ of the form $l_{m}(a)=m a-a m(a \in A)$, which are called inner derivations. Thus $H^{1}(A, M)$ is the quotient of the space of derivations by inner derivations.

Interpretation of $H H^{2}(A)$ and $H H^{3}(A)$ : deformation theory.

Now let $M=A$ be the algebra itself.

- An infinitesimal deformation of $A$ is an associative $\epsilon$-linear product $*$ on $A[\epsilon] / \epsilon^{2}$ such that $a * b=a b \bmod \epsilon$. This last condition means that for any $a, b \in A, a * b=a b+\mu(a, b) \epsilon$, with $\mu: A \otimes A \rightarrow A$. The associativity of $*$ is then equivalent to

$$
a \mu(b, c)+\mu(a, b c)=\mu(a, b) c+\mu(a b, c)
$$

which is exactly the 2-cocycle condition. Conversely, any 2-cocycle allows us to define an infinitesimal deformation of $A$

Two infinitesimal deformations $*$ and $*^{\prime}$ are equivalent if there is an isomorphism of $k[\epsilon] / \epsilon^{2}$ algebras $\left(A[\epsilon] / \epsilon^{2}, *\right) \rightarrow\left(A[\epsilon] / \epsilon^{2}, *^{\prime}\right)$ that is the identity $\bmod \epsilon$. This last condition means that there exists $l: A \rightarrow A$ such that the isomorphism maps $a$ to $a+l(a) \epsilon$. Being a morphism is then equivalent to

$$
\mu(a, b)+l(a b)=\mu^{\prime}(a, b)+a l(b)+l(a) b
$$

which is equivalent to $\mu-\mu^{\prime}=d_{H}(l)$

Therefore $H H^{2}(A)$ is the set of infinitesimal deformations of $A$ up to equivalences. 
- An order $n(n>0)$ deformation of $A$ is an associative $\epsilon$-linear product $*$ on $A[\epsilon] / \epsilon^{n+1}$ such that $a * b=a b \bmod \epsilon$. This last condition means that the product is given by

$$
a * b=a b+\sum_{i=1}^{n} \epsilon^{i} \mu_{i}(a, b),
$$

with $\mu_{i}: A \otimes A \rightarrow A$. Let us define $\mu:=\sum_{i=1}^{n} \mu_{i} \epsilon^{i} \in C^{2}(A, A[\epsilon])$. The associativity is then equivalent to

$$
d_{H}(\mu)(a, b, c)=\mu(\mu(a, b), c)-\mu(a, \mu(b, c)) \bmod \epsilon^{n+1}
$$

Proposition 2.1 (Gerstenhaber,[16]). If $*$ is an order $n$ deformation then the linear map $\nu_{n+1}: A^{\otimes 3} \rightarrow A$ defined by

$$
\nu_{n+1}(a, b, c):=\sum_{i=1}^{n}\left(\mu_{i}\left(\mu_{n+1-i}(a, b), c\right)-\mu_{i}\left(a, \mu_{n+1-i}(b, c)\right)\right)
$$

is a 3-cocyle: $d_{H}\left(\nu_{n+1}\right)=0$.

Proof. Let us define $\nu(a, b, c):=\mu(\mu(a, b), c)-\mu(a, \mu(b, c)) \in A[\epsilon]$. The associativity condition then reads $d_{H}(\mu)=\nu \bmod \epsilon^{n+1}$ and $\nu_{n+1}$ is precisely the coefficient of $\epsilon^{n+1}$ in $\nu$. Therefore it remains to prove that $d_{H}(\nu)=0 \bmod \epsilon^{n+2}$.

We let as an exercise to prove that

$$
\begin{array}{r}
d_{H}(\nu)(a, b, c, d)=\mu\left(a, d_{H}(\mu)(b, c, d)\right)-d_{H}(\mu)(\mu(a, b), c, d)+d_{H}(\mu)(a, \mu(b, c), d) \\
-d_{H}(\mu)(a, b, \mu(c, d))+\mu\left(d_{H}(\mu)(a, b, c), d\right)
\end{array}
$$

Then it follows from the associativity condition that $\bmod \epsilon^{n+2}$ the l.h.s. equals

$$
\nu(\mu(a, b), c, d)-\nu(a, \mu(b, c), d)+\nu(a, b, \mu(c, d))-\mu(\nu(a, b, c), d)+\mu(a, \nu(b, c, d)) .
$$

Finally, a straightforward computation shows that this last expression is identically zero.

Given an order $n$ deformation one can ask if it is possible to extend it to an order $n+1$ deformation. This means that we ask for a linear map $\mu_{n+1}: A \otimes A \rightarrow A$ such that

$$
\sum_{i=0}^{n+1} \mu_{i}\left(\mu_{n+1-i}(a, b), c\right)=\sum_{i=0}^{n+1} \mu_{i}\left(a, \mu_{n+1-i}(b, c)\right),
$$

which is equivalent to $d_{H}\left(\mu_{n+1}\right)=\nu_{n+1}$.

In other words, the only obstruction for extending deformations lies in $H H^{3}(A)$.

This deformation-theoretical interpretation of Hochschild cohomology is due to M. Gerstenhaber [16].

\subsection{Spectral sequences.}

Spectral sequences are essential algebraic tools for working with cohomology. They were invented by J. Leray [24, 25].

\section{Definition.}

A spectral sequence is a sequence $\left(E_{r}, d_{r}\right)_{r \geq 0}$ of bigraded spaces

$$
E_{r}=\bigoplus_{(p, q) \in \mathbb{Z}^{2}} E_{r}^{p, q}
$$

together with differentials

$$
d_{r}: E_{r}^{p, q} \longrightarrow E_{r}^{p+r, q-r+1}, \quad d_{r} \circ d_{r}=0
$$

such that $H\left(E_{r}, d_{r}\right)=E_{r+1}$ (as bigraded spaces).

One says that a spectral sequence converges (to $E_{\infty}$ ) or stabilizes if for any $(p, q)$ there exists $r(p, q)$ such that for all $r \geq r(p, q), E_{r}^{p, q}=E_{r(p, q)}^{p, q}$. We then define $E_{\infty}^{p, q}:=E_{r(p, q)}^{p, q}$. It happens when $d_{r}^{p+r, q-r+1}=d_{r}^{p, q}=0$ for $r \geq r(p, q)$. 
A convenient way to think about spectral sequences is to draw them :

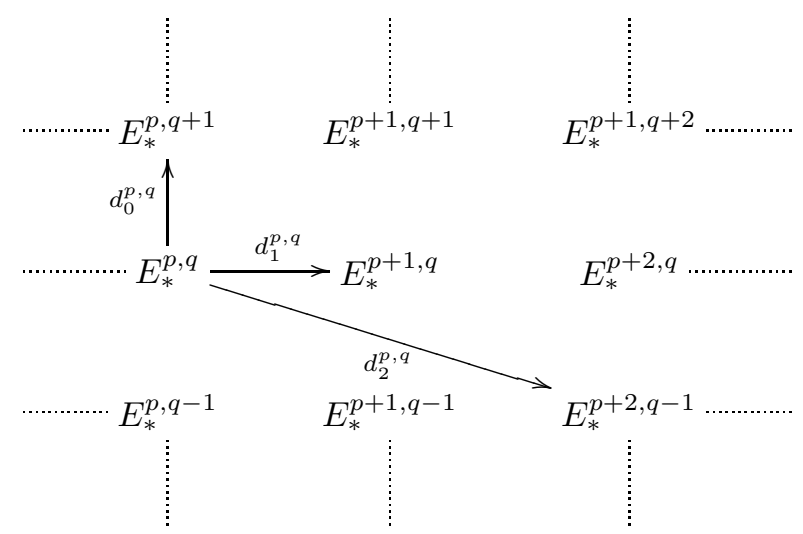

The spectral sequence of a filtered complex.

A filtered complex is a decreasing sequence of complexes

$$
C^{\bullet}=F^{0} C^{\bullet} \supset \cdots \supset F^{p} C^{\bullet} \supset F^{p+1} C^{\bullet} \supset \cdots \supset \bigcap_{i \in \mathbb{N}} F^{i} C^{\bullet}=\{0\} .
$$

Here we have assumed that the filtration is separated $\left(\cap_{p} F^{p} C^{n}=\{0\}\right.$ for any $\left.n \in \mathbb{Z}\right)$.

Let us construct a spectral sequence associated to a filtered complex $\left(F^{*} C^{\bullet}, d\right)$. We first define

$$
E_{0}^{p, q}:=\operatorname{Gr}^{p}\left(C^{p+q}\right)=\frac{F^{p} C^{p+q}}{F^{p+1} C^{p+q}}
$$

and $d_{0}=d: E_{0}^{p, q} \rightarrow E_{0}^{p, q+1}$. $d_{0}$ is well-defined since $d\left(F^{p+1} C^{p+q}\right) \subset F^{p+1} C^{p+q+1}$.

We then define

$$
E_{1}^{p, q}:=H^{p+q}\left(\operatorname{Gr}^{p}\left(C^{p+q}\right)\right)=\frac{\left\{a \in F^{p} C^{p+q} \mid d(a) \in F^{p+1} C^{p+q+1}\right\}}{d\left(F^{p} C^{p+q-1}\right)+F^{p+1} C^{p+q}}
$$

and $d_{1}=d: E_{1}^{p, q} \rightarrow E_{1}^{p+1, q}$.

More generally we define

$$
E_{r}^{p, q}:=\frac{\left\{a \in F^{p} C^{p+q} \mid d(a) \in F^{p+r} C^{p+q+1}\right\}}{d\left(F^{p-r+1} C^{p+q-1}\right)+F^{p+1} C^{p+q}}
$$

and $d_{r}=d: E_{r}^{p, q} \rightarrow E_{r}^{p+r, q-r+1}$. Here the denominator is implicitely understood as $\{$ denominator as written $\} \cap\{$ numerator $\}$.

Exercise 2.2. Prove that $H\left(E_{r}, d_{r}\right)=E_{r+1}$.

We now have the following:

Proposition 2.3. If the spectral sequence $\left(E_{r}\right)_{r}$ associated to a filtered complex $\left(F^{*} C^{\bullet}, d\right)$ converges then

$$
E_{\infty}^{p, q}=\operatorname{Gr}^{p} H^{p+q}\left(C^{\bullet}\right)
$$

Proof. Let $(p, q) \in \mathbb{Z}^{2}$. For $r \geq \max (r(p, q), p+1)$,

$$
\begin{aligned}
E_{r}^{p, q} & =\frac{\left\{a \in F^{p} C^{p+q} \mid d(a)=0\right\}}{d\left(C^{p+q-1}\right)+F^{p+1} C^{p+q}} \\
& =\frac{F^{p} H^{p+q}\left(C^{\bullet}\right)}{F^{p+1} H^{p+q}\left(C^{\bullet}\right)}=\operatorname{Gr}^{p} H^{p+q}\left(C^{\bullet}\right) .
\end{aligned}
$$


This proves the proposition.

Example 2.4 (Spectral sequences of a double complex). Assume we are given a double complex $\left(C^{\bullet \bullet \bullet}, d, d^{\prime}\right)$, i.e. a $\mathbb{Z}^{2}$-graded vector space together with degree $(1,0)$ and $(0,1)$ linear maps $d^{\prime}$ and $d^{\prime \prime}$ such that $d^{\prime} \circ d^{\prime}=0, d^{\prime \prime} \circ d^{\prime \prime}=0$ and $d^{\prime} \circ d^{\prime \prime}+d^{\prime \prime} \circ d^{\prime}=0$. Then the total complex $\left(C_{t o t}^{\bullet}, d_{t o t}\right)$ is defined as

$$
C_{t o t}^{n}:=\bigoplus_{p+q=n} C^{p, q}, \quad d_{t o t}:=d^{\prime}+d^{\prime \prime} .
$$

There are two filtrations, and thus two spectral sequences, naturally associated to $\left(C_{t o t}^{\bullet}, d_{t o t}\right)$ :

$$
F^{\prime k} C_{t o t}^{n}:=\bigoplus_{\substack{p+q=n \\ q \geq k}} C^{p, q} \quad \text { and } \quad{F^{\prime \prime}}^{k} C_{t o t}^{n}:=\bigoplus_{\substack{p+q=n \\ p \geq k}} C^{p, q} .
$$

Therefore the first terms of the corresponding spectral sequences are:

$$
\begin{aligned}
& E^{\prime p, q}=H^{q}\left(C^{\bullet, p}, d^{\prime}\right) \quad \text { with } \quad d_{1}=d^{\prime \prime} \\
& E^{\prime \prime p, q}=H^{q}\left(C^{p, \bullet}, d^{\prime \prime}\right) \text { with } d_{1}=d^{\prime} .
\end{aligned}
$$

In the case the $d^{\prime}$-cohomology is concentrated in only one degree $q$ then the spectral sequence stabilizes at $E_{2}$ and the total cohomology is given by $H_{t o t}^{\bullet}=H^{\bullet-q}\left(H^{q}\left(C, d^{\prime}\right), d^{\prime \prime}\right)$.

Spectral sequences of algebras.

A spectral sequence of algebras is a spectral sequence such that each $E_{r}$ is equipped with a bigraded associative product that turns $\left(E_{r}, d_{r}\right)$ into a DG algebra. Of course, we require that $H\left(E_{r}, d_{r}\right)=E_{r+1}$ as algebras.

As in the previous paragraph a filtered DG algebra $\left(F^{*} A^{\bullet}, d\right)$ gives rise to a spectral sequence of algebras $\left(E_{r}\right)_{r}$ such that

- $E_{0}^{p, q}:=\operatorname{Gr}^{p}\left(A^{p+q}\right)$,

- $E_{1}^{p, q}:=H^{p+q}\left(\operatorname{Gr}^{p}\left(A^{p+q}\right)\right)$,

- if it converges then $E_{\infty}^{p, q}=\mathrm{Gr}^{p} H^{p+q}\left(A^{\bullet}\right)$.

\subsection{Application: Chevalley-Eilenberg vs Hochschild cohomolgy.}

Let $M$ be a $U(\mathfrak{g})$-bimodule. Then $M$ is equipped with a $\mathfrak{g}$-module structure given as follows:

$$
\forall x \in \mathfrak{g}, \forall m \in M, x \cdot m=x m-m x .
$$

We want to prove the following

Theorem 2.5. 1. There is an isomorphism $H^{\bullet}(\mathfrak{g}, M) \cong H^{\bullet}(U(\mathfrak{g}), M)$.

2. If $M=A$ is equipped with a $U(\mathfrak{g})$-invariant associative product then the previous isomorphism becomes an isomorphism of (graded) algebras.

We define a filtration on the Hochschild complex $C^{\bullet}(U(\mathfrak{g}), M): F^{p} C^{n}(U(\mathfrak{g}), M)$ is given by linear maps $U(\mathfrak{g})^{\otimes n} \rightarrow M$ that vanish on

$$
\bigoplus_{i_{1}+\cdots+i_{n}<p} U(\mathfrak{g})_{\leq i_{1}} \otimes \cdots \otimes U(\mathfrak{g})_{\leq i_{n}} .
$$

Computing $E_{0}$.

First of all it follows from the PBW theorem that

$$
E_{0}^{p, q}=G r^{p}\left(C^{p+q}(U(\mathfrak{g}), M)\right)=\bigoplus_{i_{1}+\cdots+i_{p+q}=p} \operatorname{Lin}\left(S^{i_{1}}(\mathfrak{g}) \otimes \cdots \otimes S^{i_{p+q}}(\mathfrak{g}), M\right) .
$$

We then compute $d_{0}$. 
Let $P \in F^{p} C^{p+q}(U(\mathfrak{g}), M), j_{0}+\cdots+j_{p+q}=p$ and $x_{0}, \ldots, x_{p+q} \in \mathfrak{g}$. We have

$$
\begin{aligned}
d_{H}(P)\left(x_{0}^{j_{0}}, \ldots, x_{p+q}^{j_{p+q}}\right)= & x_{0}^{j_{0}} P\left(x_{1}^{j_{1}}, \ldots, x_{p+q}^{j_{p+q}}\right)+\sum_{k=1}^{p+q}(-1)^{k} P\left(x_{0}^{j_{0}}, \ldots, x_{k-1}^{j_{k-1}} * x_{k}^{j_{k}}, \ldots, x_{p+q}^{j_{p+q}}\right) \\
& +(-1)^{p+q+1} P\left(x_{0}^{j_{0}}, \ldots, x_{p+q-1}^{j_{p+q}}\right) x_{p+q}^{j_{p+q}} \\
= & \epsilon\left(x_{0}^{j_{0}}\right) P\left(x_{1}^{j_{1}}, \ldots, x_{p+q}^{j_{p+q}}\right)+\sum_{k=1}^{p+q}(-1)^{k} P\left(x_{0}^{j_{0}}, \ldots, x_{k-1}^{j_{k-1}} x_{k}^{j_{k}}, \ldots, x_{p+q}^{j_{p+q}}\right) \\
& +(-1)^{p+q+1} P\left(x_{0}^{j_{0}}, \ldots, x_{p+q-1}^{j_{p+q}}\right) \epsilon\left(x_{p+q}^{j_{p+q}}\right),
\end{aligned}
$$

where $\epsilon: S(\mathfrak{g}) \rightarrow k$ is the projection on degree 0 elements. Therefore $d_{0}$ is the coboundary operator for the Hochschild cohomology of $\widehat{S}(\mathfrak{g})$ with values in the bimodule $M$ (where the left and right action coincide and are given by $\epsilon$ ).

\section{Computing $E_{1}$.}

We first need to compute $H(\widehat{S}(\mathfrak{g}), M)=H(\widehat{S}(\mathfrak{g}), k) \otimes M$. For this we will need a standard lemma from homological algebra: one can define an inclusion of complexes $\left(\wedge^{\bullet}(\mathfrak{g})^{*}, 0\right) \hookrightarrow$ $C \bullet(\widehat{S}(\mathfrak{g}), k)$ as the transpose of the composed map

$$
\otimes^{n} S(\mathfrak{g}) \longrightarrow \otimes^{n} \mathfrak{g} \longrightarrow \wedge^{n} \mathfrak{g}
$$

We therefore need the following standard result of homological algebra:

Lemma 2.6. Let $V$ be a vector space. Then the inclusion $\left(\wedge^{\bullet}\left(V^{*}\right), 0\right) \hookrightarrow C^{\bullet}(\widehat{S}(V), k)$, resp. the projection $C^{\bullet}(\widehat{S}(V), k) \rightarrow\left(\wedge^{\bullet}\left(V^{*}\right), 0\right)$, is a quasi-isomorphism of complexes that induces a (graded) algebra isomorphism $\wedge^{\bullet}(V)^{*} \cong H^{\bullet}(\widehat{S}(V), k)$ on the level of cohomology, resp. a quasi-isomorphism of $D G$ algebras.

Sketch of the proof. First observe that elements of $T^{\bullet}\left(V^{*}\right)$ are Hochschild cocycles in $C^{\bullet}(\widehat{S}(V), k)$. We then let as an exercise to prove that Hochschild cocycles lying in the kernel of the surjective graded algebra morphism $p: C^{\bullet}(\widehat{S}(V), k) \rightarrow T^{\bullet}\left(V^{*}\right)$ are coboundaries. Consequently, $H^{\bullet}(\widehat{S}(V), k)$ is given by the quotient of the tensor algebra $T\left(V^{*}\right)$ by the two-sided ideal generated by the image of $p \circ d_{H}$. The only non-trivial elements in the image of $p \circ d_{H}$ are

$$
p \circ d_{H}\left(x_{1} \otimes \cdots \otimes x_{i} x_{i+1} \otimes \cdots \otimes x_{n}\right)=x_{1} \otimes \cdots \otimes\left(x_{i} \otimes x_{i+1}+x_{i+1} \otimes x_{i}\right) \otimes \cdots \otimes x_{n} .
$$

Therefore $H^{\bullet}(\widehat{S}(V), k) \cong T^{\bullet}\left(V^{*}\right) /\langle x \otimes y+y \otimes x \mid x, y \in V\rangle=S^{\bullet}\left(V^{*}\right)$.

Using the previous lemma one has that

$$
E_{1}^{p, q}= \begin{cases}\operatorname{Lin}\left(\wedge^{p}(\mathfrak{g}), M\right) & \text { if } q=0 \\ \{0\} & \text { otherwise. }\end{cases}
$$

Therefore we have that the spectral sequence converges and $E_{\infty}=E_{2}=H\left(E_{1}, d_{1}\right)$. It thus remains to prove that $d_{1}=d_{C}$.

We know prove that $d_{1}$ is the Chevalley-Eilenberg differential. It suffices to prove this on degree 0 and 1 elements:

$$
d_{1}(m)(y)=d_{H}(m)(y)=y m-m y=d_{C}(m)(y)
$$

and

$$
\begin{aligned}
d_{1}(l)(x, y) & =\frac{1}{2}\left(d_{H}(l)(x, y)-d_{H}(l)(y, x)\right)=\frac{1}{2}(x l(y)-l(x y)+l(x) y-y l(x)+l(y x)-l(y) x) \\
& =\frac{1}{2}(x \cdot l(y)-y \cdot l(x)-l([x, y]))=\frac{1}{2}\left(d_{C}(l)(x, y)\right)
\end{aligned}
$$


This ends the proof of the first part of Theorem 2.5: $H^{\bullet}(U(\mathfrak{g}), M)=E_{2}=H^{\bullet}(\mathfrak{g}, M)$.

The second part of the theorem follows from the fact that $H^{\bullet}(U(\mathfrak{g}), A)$ is isomorphic to its associated graded as an algebra. 


\section{Dolbeault cohomology and the Kontsevich isomorphism}

The main goal of this section is to present an analogous statement, for complex manifolds, of the Duflo theorem. It was proposed by M. Kontsevich in his seminal paper [22]. We first begin with a crash course in complex geometry (mainly its algebraic aspect) and then define the Atiyah and Todd classes, which play a rôle analogous to the adjoint action and Duflo element, respectively. We continue with the definition of the Hochschild cohomology of a complex manifold and state the result.

Throughout this Section $k=\mathbb{C}$ is the field of complex numbers.

\subsection{Complex manifolds.}

An almost complex manifold is a differentiable manifold $M$ together with an automorphism $J: T M \rightarrow T M$ of its tangent bundle such that $J^{2}=-\mathrm{id}$. In particular it is even dimensional. Then the complexified tangent bundle $T_{\mathbb{C}} M=T M \otimes \mathbb{C}$ decomposes as the direct sum $T^{\prime} \oplus T^{\prime \prime}$ of two eigenbundles corresponding to the eigenvalues \pm i of $J$.

A complex manifold is an almost complex manifold $(M, J)$ that is integrable, i.e. such that one of the following equivalent conditions is satisfied:

- $T^{\prime}$ is stable under the Lie bracket,

- $T^{\prime \prime}$ is stable under the Lie bracket.

Sections of $T^{\prime}$ (resp. $\left.T^{\prime \prime}\right)$ are called vector fields of type $(1,0)$ (resp. of type $(0,1)$ ).

The graded space $\Omega^{\bullet}(M)=\Gamma\left(M, \wedge^{\bullet} T_{\mathbb{C}}^{*} M\right)$ of complex-valued differential forms therefore becomes a bigraded space. Namely

$$
\Omega^{p, q}(M)=\Gamma\left(M, \wedge^{p}\left(T^{\prime}\right)^{*} \otimes \wedge^{q}\left(T^{\prime \prime}\right)^{*}\right) .
$$

For any $\omega \in \Omega^{p, q}(M)$ one has that

$$
\mathrm{d} \omega \in \Gamma\left(M,\left(\wedge^{p}\left(T^{\prime}\right)^{*} \otimes \wedge^{q}\left(T^{\prime \prime}\right)^{*}\right) \wedge T_{\mathbb{C}}^{*} M\right)=\Omega^{p+1, q}(M) \oplus \Omega^{p, q+1}(M),
$$

therefore $\mathrm{d}=\partial+\bar{\partial}$ with $\partial: \Omega^{\bullet}, \bullet(M) \rightarrow \Omega^{\bullet+1, \bullet}(M)$ and $\bar{\partial}: \Omega^{\bullet \bullet \bullet}(M) \rightarrow \Omega^{\bullet \bullet \bullet+1}(M)$. The integrability condition ensures that $\bar{\partial} \circ \bar{\partial}=0$ (it is actually equivalent). Therefore one can define a DG algebra $\left(\Omega^{0, \bullet}(M), \bar{\partial}\right)$, the Dolbeault algebra.

The corresponding cohomology is denoted $H_{\bar{\partial}}^{\bullet}(M)$.

Let $E$ be a differentiable $\mathbb{C}$-vector bundle (i.e. fibers are $\mathbb{C}$-vector spaces). The space $\Omega(M, E)$ of forms with values in $E$ is bigraded as above. In general one can NOT turn $\Omega^{0, \bullet}(M, E)$ into a DG vector space with differential $\bar{\partial}$ extending the one on $\Omega^{0, \bullet}(M)$ in the following way: for any $\xi \in \Omega^{0, \bullet}(M)$ and any $s \in \Gamma(M, E)$

$$
\bar{\partial}(\xi s)=(\bar{\partial} \xi) s+(-1)^{|\xi|} \xi \bar{\partial}(s) .
$$

Such a differential is called a $\bar{\partial}$-connection and it is uniquely determined by its restriction on degree zero elements

$$
\bar{\partial}: \Gamma(M, E) \longrightarrow \Omega^{0,1}(M, E) .
$$

A complex vector bundle $E$ equipped with a $\bar{\partial}$-connection is called a holomorphic vector bundle. Therefore, given a holomorphic vector bundle $E$ one has an associated Dolbeault cohomology $H_{\grave{\partial}}^{\bullet}(M, E)$.

For a comprehensible introduction to complex manifolds we refer to the first chapters of the standard monography [17]. 
Interpretation of $H_{\bar{\partial}}^{0}(M, E)$.

There is an alternative (but equivalent) definition of complex manifolds: a complex manifold is a topological space locally homeomorphic to $\mathbb{C}^{n}$ and such that transition functions are biholomorphic.

In this framework, in local holomorphic coordinates $\left(z^{1}, \ldots, z^{n}\right)$ one has $\partial=\mathrm{d} z^{i} \frac{\partial}{\partial z^{i}}$, $\bar{\partial}=\mathrm{d} \bar{z}^{i} \frac{\partial}{\partial \bar{z}^{i}}$, and $J$ is simply given by complex conjugation. Therefore a holomorphic function, i.e. a function that is holomorphic in any chart of holomorphic coordinates, is a $C^{\infty}$ function $f$ satisfying $\bar{\partial}(f)=0$.

Similarly, a holomorphic vector bundle is locally homeomorphic to $\mathbb{C}^{n} \times V$ ( $V$ is the typical fiber) with transition functions being $\operatorname{End}(V)$-valued holomorphic functions. Again one can locally write $\bar{\partial}=\mathrm{d} z^{i} \frac{\partial}{\partial \bar{z}^{i}}$ and holomorphic sections, i.e. sections that are holomorphic in small enough charts, are $C^{\infty}$ sections $s$ such that $\bar{\partial}(s)=0$.

In other words, the 0 -th Dolbeault cohomology $H_{\bar{\partial}}^{0}(M, E)$ of a holomorphic vector bundle $E$ is its space of holomorphic sections.

Interpretation of $H_{\bar{\partial}}^{1}(M, \operatorname{End}(E))$.

Let $E$ be a $C^{\infty}$ vector bundle.

Observe that given two $\bar{\partial}$-connections $\bar{\partial}_{1}$ and $\bar{\partial}_{2}$, their difference $\xi=\bar{\partial}_{2}-\bar{\partial}_{1}$ lies in $\Omega^{0,1}(M, \operatorname{End}(E))\left(\right.$ since $\left.\bar{\partial}_{i}(f s)=\bar{\partial}(f) s+f \bar{\partial}_{i}(s)\right)$. Therefore the integrability condition $\bar{\partial}_{i} \circ \bar{\partial}_{i}=0$ implies that $\bar{\partial}_{1} \circ \xi+\xi \circ \bar{\partial}_{1}+\xi \circ \xi=0$. Therefore any infinitesimal deformation $\bar{\partial}_{\epsilon}$ of a holomorphic structure $\bar{\partial}$ on $E$ (i.e. a $\mathbb{C}[\epsilon] / \epsilon^{2}$-valued $\bar{\partial}$-connection $\bar{\partial}_{\epsilon}=\bar{\partial} \bmod \epsilon$ ) can be written as $\bar{\partial}_{\epsilon}=\bar{\partial}+\epsilon \xi$ with $\xi \in \Omega^{0,1}(M, \operatorname{End}(E))$ satisfying $\bar{\partial} \circ \xi+\xi \circ \bar{\partial}=0$.

Such an infinitesimal deformation is trivial, meaning that it identifies with $\bar{\partial}$ under an automorphism of $E$ (over $\mathbb{C}[\epsilon] / \epsilon^{2}$ ) that is the identity $\bmod \epsilon$, if and only if there exists a section $s$ of $\operatorname{End}(E)$ such that $\xi=\bar{\partial} \circ s-s \circ \bar{\partial}$.

Consequently the space of infinitesimal deformations of the holomorphic structure of $E$ up to the trivial ones is given by $H_{\bar{\partial}}^{1}(M, \operatorname{End}(E))$.

Remark 3.1. Here we should emphazise the following obvious facts we implicitely use.

First of all, if $E$ is a holomorphic vector bundle then so is $E^{*}$. Namely, for any $s \in \Gamma(M, E)$ and $\zeta \in \Gamma\left(M, E^{*}\right)$ one defines $\langle\bar{\partial}(\zeta), s\rangle:=\bar{\partial}(\langle\zeta, s\rangle)-\langle\zeta, \bar{\partial}(s)\rangle$.

Then, if $E_{1}$ and $E_{2}$ are holomorphic vector bundles then so is $E_{1} \otimes E_{2}$ : for any $s_{i} \in$ $\Gamma\left(M, E_{i}\right)(i=1,2) \bar{\partial}\left(s_{1} \otimes s_{2}\right):=\bar{\partial}\left(s_{1}\right) \otimes s_{2}+s_{1} \otimes \bar{\partial}\left(s_{2}\right)$.

Thus, if $E$ is a holomorphic vector bundle then so is $\operatorname{End}(E)=E^{*} \otimes E$ : for any $s \in$ $\Gamma(\operatorname{End}(E))$ one has $\bar{\partial}(s)=\bar{\partial} \circ s-s \circ \bar{\partial}$.

\subsection{Atiyah and Todd classes.}

Let $E \rightarrow M$ be a holomorphic vector bundle. In this paragraph we introduce Atiyah and Todd classes of $E$. Any connection $\nabla$ on $M$ with values in $E$, i.e. a linear operator

$$
\nabla: \Gamma(M, E) \longrightarrow \Omega^{1}(M, E)
$$

satisfying the Leibniz rule $\nabla(f s)=(\mathrm{d} f) s+f(\nabla s)$, decomposes as $\nabla=\nabla^{\prime}+\nabla^{\prime \prime}$, where $\nabla^{\prime}$ (resp. $\left.\nabla^{\prime \prime}\right)$ takes values in $\Omega^{1,0}(M, E)\left(\right.$ resp. $\left.\Omega^{0,1}(M, E)\right)$. Connections such that $\nabla^{\prime \prime}=\bar{\partial}$ are said compatible with the complex structure.

A connection compatible with the complex structure always exists. Namely, it always exists locally and one can then use a partition of unity to conclude. Let us choose such a connection $\nabla$ and consider its curvature $R \in \Omega^{2}(M, \operatorname{End}(E))$ : for any $u, v \in \mathfrak{X}(M)$

$$
R(u, v)=\nabla_{u} \nabla_{v}-\nabla_{v} \nabla_{u}-\nabla_{[u, v]} .
$$

In other words $\nabla \circ \nabla=R$.

One can see that in the case of a connection compatible with the complex structure the curvature tensor does not have $(0,2)$-component: $R=R^{2,0}+R^{1,1}$. 
Remember that locally a connection can be written $\nabla=\mathrm{d}+\Gamma$, with $\Gamma \in \Omega^{1}\left(U, \operatorname{End}\left(E_{\mid U}\right)\right)$. The compatibility with the complex structure imposes that $\Gamma \in \Omega^{1,0}\left(U, \operatorname{End}\left(E_{\mid U}\right)\right)$. Then one can check easily that $R^{1,1}=\bar{\partial}(\Gamma)$ (locally!). Therefore $\bar{\partial}\left(R^{1,1}\right)=0$. We define the Atiyah class of $E$ as the Dolbeault class

$$
\operatorname{at}_{E}:=\left[R^{1,1}\right] \in H_{\bar{\partial}}^{1}\left(\left(T^{\prime}\right)^{*} \otimes(\operatorname{End}(E))\right) .
$$

Lemma 3.2. at $_{E}$ is independent of the choice of a connection compatible with the complex structure.

Proof. Let $\nabla$ and $\widetilde{\nabla}$ be two such connections. We see that $\nabla-\widetilde{\nabla}$ is a 1 -form with values in $\operatorname{End}(E)$ : for any $f \in C^{\infty}(M)$ and $s \in \Gamma(M, E)$

$$
(\nabla-\widetilde{\nabla})(f s)=(\mathrm{d} f) s+f(\nabla s)-(\mathrm{d} f) s-f(\widetilde{\nabla} s)=f(\nabla-\widetilde{\nabla})(s) .
$$

Therefore $\Gamma-\widetilde{\Gamma}$ is a globally well-defined tensor and $R^{1,1}-\widetilde{R}^{1,1}=\bar{\partial}(\Gamma-\widetilde{\Gamma})$ is a Dolbeault coboundary.

For any $n>0$ one defines the $n$-th scalar Atiyah class $a_{n}(E)$ as

$$
a_{n}(E):=\operatorname{tr}\left(\operatorname{at}_{E}^{n}\right) \in H_{\bar{\partial}}^{n}\left(M, \wedge^{n}\left(T^{\prime}\right)^{*}\right) .
$$

Observe that $\operatorname{tr}\left(\left(R^{1,1}\right)^{n}\right)$ lies in $\Omega^{0, n}\left(M, \otimes^{n}\left(T^{\prime}\right)^{*}\right)$, but we regard it as an element in $\Omega^{0, n}\left(M, \wedge^{n}\left(T^{\prime}\right)^{*}\right)$ thanks to the natural projection $\otimes\left(T^{\prime}\right)^{*} \rightarrow \wedge\left(T^{\prime}\right)^{*}$.

The Todd class of $E$ is then

$$
\operatorname{td}_{E}:=\operatorname{det}\left(\frac{\mathrm{at}_{E}}{1-e^{-\mathrm{at}_{E}}}\right)
$$

One sees without difficulties that it can be expanded formally in terms of $a_{n}(E)$.

\subsection{Hochschild cohomology of a complex manifold.}

Hochschild cohomology of a differentiable manifold.

Let $M$ be a differentiable manifold. We introduce the differential graded algebras $T_{\text {poly }}^{\bullet} M$ and $D_{\text {poly }}^{\bullet} M$ of polyvector field and polydifferential operators on $M$.

First of all $T_{\text {poly }}^{\bullet} M:=\Gamma(M, \wedge \bullet T M)$ with product $\wedge$ and differential $d=0$.

The algebra of differential operators is the subalgebra of $\operatorname{End}\left(C^{\infty}(M)\right)$ generated by functions and vector fields. Then we define the DG algebra $D_{\text {poly }}^{\bullet} M$ as the DG subalgebra of $\left(C^{\bullet}\left(C^{\infty}(M), C^{\infty}(M)\right), \cup, d_{H}\right)$ whose elements are cochains being differential operators in each argument (i.e. if we fix all the other arguments then it is a differential operator in the remaining one).

The following result, due to J. Vey [33] (see also [22]), computes the cohomology of $D_{\text {poly }}^{\bullet} M$. It is an analogue for smooth functions of the original Hochschild-Kostant-Rosenberg theorem [19] for regular affine algebras.

Theorem 3.3. The degree 0 graded map

$$
\begin{aligned}
I_{H K R}:\left(T_{\text {poly }}^{\bullet} M, 0\right) & \longrightarrow\left(D_{\text {poly }}^{\bullet} M, d_{H}\right) \\
v_{1} \wedge \cdots \wedge v_{n} & \longmapsto\left(f_{1} \otimes \cdots \otimes f_{n} \mapsto \frac{1}{n !} \sum_{\sigma \in \mathfrak{S}_{n}}(-1)^{\sigma} v_{\sigma(1)}\left(f_{1}\right) \cdots v_{\sigma(n)}\left(f_{n}\right)\right)
\end{aligned}
$$

is a quasi-isomorphism of complexes that induces an isomorphism of (graded) algebras on the level of cohomology. 
Proof. First of all it is easy to check that it is a morphism of complexes (i.e. images of $I_{H K R}$ are cocycles).

Then one can see that everything is $C^{\infty}(M)$-linear: the products $\wedge$ and $\cup$, the differential $d_{H}$ and the map $I_{H K R}$. Moreover, one can see that $D_{\text {poly }}^{\bullet}$ is nothing but the Hochschild complex of the algebra $J_{M}^{\infty}$ of $\infty$-jets of functions on $M$ with values in $C^{\infty}(M){ }^{4}$

As an algebra $J_{M}^{\infty}$ can be identified (non canonically) with global sections of the bundle of algebras $\widehat{S}\left(T^{*} M\right)$, and $\epsilon$ with the projection on degree 0 elements. Therefore the statement follows immediatly if one applies Lemma 2.6 fiberwise to $V=T_{m}^{*} M(m \in M)$.

Hochschild cohomology of a complex manifold.

Let us now go back to the case of a complex manifold $M$.

First of all for any vector bundle $E$ over $M$ we define $T_{\text {poly }}^{\bullet \bullet}(M, E):=\Gamma\left(M, E \otimes \wedge^{\bullet} T^{\prime}\right)$.

Then we define $\partial$-differential operators as endomorphisms of $C^{\infty}(M)$ generated by functions and type $(1,0)$ vector fields, and for any vector bundle $E$ we define $E$-valued $\partial$ differential operators as linear maps $C^{\infty}(M) \rightarrow \Gamma(M, E)$ obtained by composing $\partial$-differential operators with sections of $E$ or $T^{\prime} \otimes E$ (sections of $T^{\prime} \otimes E$ are $E$-valued type $(1,0)$ vector fields).

The complex $D_{\text {poly }}^{\prime \bullet}(M, E)$ of $E$-valued $\partial$-polydifferential operators is defined as the subcomplex of $\left(C^{\bullet}\left(C^{\infty}(M), \Gamma(M, E)\right), d_{H}\right)$ consisting of cochains that are $\partial$-differential operators in each argument.

We have the following obvious analogue of Theorem 3.3:

Theorem 3.4. The degree 0 graded map

$$
\begin{aligned}
I_{H K R}:\left(T_{\text {poly }}^{\prime \bullet}(M, E), 0\right) & \longrightarrow\left(D_{\text {poly }}^{\prime \bullet}(M, E), d_{H}\right) \\
\left(v_{1} \wedge \cdots \wedge v_{n}\right) \otimes s & \longmapsto\left(f_{1} \otimes \cdots \otimes f_{n} \mapsto \frac{1}{n !} \sum_{\sigma \in \mathfrak{S}_{n}}(-1)^{\sigma} v_{\sigma(1)}\left(f_{1}\right) \cdots v_{\sigma(n)}\left(f_{n}\right) s\right)
\end{aligned}
$$

is a quasi-isomorphism of complexes.

Now observe that $\wedge^{\bullet} T^{\prime}$ is a holomorphic bundle of graded algebras with product being $\wedge$. Namely, $T^{\prime}$ has an obvious holomorphic structure: for any $v \in \Gamma\left(M, T^{\prime}\right)$ and any $f \in C^{\infty}(M)$

$$
\bar{\partial}(v)(f):=\bar{\partial}(v(f))-v(\bar{\partial}(f)) ;
$$

and it extends uniquely to a holomorphic structure on $\wedge^{\bullet} T^{\prime}$ that is a derivation with respect to the product $\wedge$ : for any $v, w \in \Gamma\left(M, T_{\text {poly }}^{\prime \bullet}\right)$

$$
\bar{\partial}(v \wedge w)=\bar{\partial}(v) \wedge w+(-1)^{|v|} v \wedge \bar{\partial}(w) .
$$

Therefore $\bar{\partial}$ turns $\Omega^{0, \bullet}\left(M, \wedge^{\bullet} T^{\prime}\right)=T_{\text {poly }}^{\prime \bullet}\left(M, \wedge \bullet\left(T^{\prime \prime}\right)^{*}\right)$ into a DG algebra.

One also has an action of $\bar{\partial}$ on $\partial$-differential operators defined in the same way: for any $f \in C^{\infty}(M)$

$$
\bar{\partial}(P)(f)=\bar{\partial}(P(f))-P(\bar{\partial}(f)) .
$$

It can be extended uniquely to a degree one derivation of the graded algebra $D_{\text {poly }}^{\prime \bullet}\left(M, \wedge{ }^{\bullet}\left(T^{\prime \prime}\right)^{*}\right)$, with product given by

$$
P \cup Q\left(f_{1}, \ldots, f_{m+n}\right)=(-1)^{m|Q|} P\left(f_{1}, \ldots, f_{m}\right) \wedge Q\left(f_{m+1}, \ldots, f_{m+n}\right),
$$

where $|\cdot|$ refers to the exterior degree.

\footnotetext{
${ }^{4}$ Recall that $J_{M}^{\infty}:=\operatorname{Hom}_{C}{ }_{(M)}\left(D_{\text {poly }}^{1} M, C^{\infty}(M)\right)$ with product given by $j_{1} \cdot j_{2}(P):=\left(j_{1} \otimes j_{2}\right)(\Delta(P)) \quad\left(j_{1}, j_{2} \in J_{M}^{\infty}, P \in D_{\text {poly }}^{1} M\right)$,

where $\Delta(P) \in D_{\text {poly }}^{2} M$ is defined by $\Delta(P)(f, g):=P(f g)$. The module structure on $C^{\infty}(M)$ is given by the projection $\epsilon: J_{M}^{\infty} \rightarrow C^{\infty}(M)$ obtained as the transpose of $C^{\infty}(M) \hookrightarrow D_{\text {poly }}^{1} M$.
} 


\subsection{The Kontsevich isomorphism.}

Theorem 3.5. The map $I_{H K R} \circ \operatorname{td}_{T^{\prime}}^{1 / 2} \cdot$ induces an isomorphism of (graded) algebras

$$
H_{\bar{\partial}}\left(\wedge T_{\text {poly }}^{\prime}\right) \stackrel{\sim}{\longrightarrow} H\left(D_{\text {poly }}^{\prime}\left(\wedge\left(T^{\prime \prime}\right)^{*}\right), d_{H}+\bar{\partial}\right)
$$

on the level of cohomology.

This result has been stated by M. Kontsevich in [22] (see also [7]) and proved in a more general context in [6].

Remark 3.6. Since $a_{1}\left(T^{\prime}\right)$ is a derivation of $H_{\bar{\partial}}\left(\wedge T_{\text {poly }}^{\prime}\right)$ then $e^{a_{1}\left(T^{\prime}\right)}$ is an algebra automorphism of $H_{\bar{\partial}}\left(\wedge T_{\text {poly }}^{\prime}\right)$. Therefore, as for the usual Duflo isomorphism (see Remark 1.3), one can replace the Todd class of $T^{\prime}$ by its modified Todd class

$$
\widetilde{\operatorname{td}}_{T^{\prime}}:=\operatorname{det}\left(\frac{\mathrm{at}_{T^{\prime}}}{e^{\mathrm{at}_{T^{\prime}} / 2}-e^{-\mathrm{at}_{T^{\prime}} / 2}}\right) \text {. }
$$




\section{SuperspaCes AND Hochschild COHOMOLOGY}

In this section we provide a short introduction to supermathematics and deduce from it a definition of the Hochschild cohomology for DG associative algebras. Moreover we prove that the Hochschild cohomology of the Chevalley algebra $\left(\wedge^{\bullet}(\mathfrak{g})^{*}, d_{C}\right)$ of a finite dimensional Lie algebra $\mathfrak{g}$ is isomorphic to the Hochschild cohomology of its universal envelopping algebra $U(\mathfrak{g})$.

\subsection{Supermathematics.}

Definition 4.1. A super vector space (simply, a superspace) is a $\mathbb{Z} / 2 \mathbb{Z}$-graded vector space $V=V_{0}+V_{1}$.

In addition to the usual well-known operations on $G$-graded vector spaces (direct sum $\oplus$, tensor product $\otimes$, spaces of linear maps $\operatorname{Hom}(-,-)$, and duality $\left.(-)^{*}\right)$ one has a parity reversion operation $\Pi$ : $(\Pi V)_{0}=V_{1}$ and $(\Pi V)_{1}=V_{0}$.

In the sequel $V$ is always a finite dimensional super vector space.

\section{Supertrace and Berezinian.}

For any endomorphism $X$ of $V$ (also refered as a supermatrix on $V$ ) one can define its supertrace str as follows: if we write $X=\left(\begin{array}{ll}x_{00} & x_{10} \\ x_{01} & x_{11}\end{array}\right)$, meaning that $X=x_{00}+x_{10}+x_{01}+x_{11}$ with $x_{i j} \in \operatorname{Hom}\left(V_{i}, V_{j}\right)$, then

$$
\operatorname{str}(X):=\operatorname{tr}\left(x_{00}\right)-\operatorname{tr}\left(x_{11}\right) .
$$

On invertible endomorphisms we also have the Berezinian Ber (or superdeterminant) which is uniquely determined by the two defining properties:

$$
\operatorname{Ber}(A B)=\operatorname{Ber}(A) \operatorname{Ber}(B) \quad \text { and } \quad \operatorname{Ber}\left(e^{X}\right)=e^{\operatorname{str}(X)} .
$$

Symmetric and exterior algebras of a super vector space.

The (graded) symmetric algebra $S(V)$ of $V$ is the quotient of the free algebra $T(V)$ generated by $V$ by the two-sided ideal generated by

$$
v \otimes w-(-1)^{|v||w|} w \otimes v .
$$

It has two different $\left(\mathbb{Z}_{-}^{-}\right)$gradings:

- the first one (by the symmetric degree) is obtained by assigning degree 1 to elements of $V$. Its degree $n$ homogeneous part, denoted by $S^{n}(V)$, is the quotient of the space $V^{\otimes n}$ by the action of the symmetric group $\mathfrak{S}_{n}$ by super-permutations:

$$
(i, i+1) \cdot\left(v_{1} \otimes \cdots \otimes v_{n}\right):=(-1)^{\left|v_{i}\right|\left|v_{i+1}\right|} v_{1} \otimes \cdots v_{i} \otimes v_{i+1} \cdots \otimes v_{n} .
$$

- the second one (the internal grading) is obtained by assigning degree $i \in\{0,1\}$ to elements of $V_{i}$. Its degree $n$ homogeneous part is denoted by $S(V)^{n}$, and we write $|x|$ for the internal degree of an element $x \in S(V)$.

Example 4.2. (a) If $V=V_{0}$ is purely even then $S(V)=S\left(V_{0}\right)$ is the ususal symmetric algebra of $V_{0}, S^{n}(V)=S^{n}\left(V_{0}\right)$ and $S(V)$ is concentrated in degree 0 for the internal grading. (b) If $V=V_{1}$ is purely odd then $S(V)=\wedge\left(V_{1}\right)$ is the exterior algebra of $V_{1}$. Moreover, $\wedge^{n}(V)=\wedge^{n}\left(V_{1}\right)=\wedge(V)^{n}$.

The (graded) exterior algebra $\wedge(V)$ of $V$ is the quotient of the free algebra $T(V)$ generated by $V$ by the two-sided ideal generated by

$$
v \otimes w+(-1)^{|v||w|} w \otimes v .
$$

It has two different $(\mathbb{Z}$-)gradings: 
- the first one (by the exterior degree) is obtained by assigning degree 1 to elements of $V$. Its degree $n$ homogeneous part is, denoted $\wedge^{n}(V)$, is the quotient of the space of $V^{\otimes n}$ by the action of the symmetric group $\mathfrak{S}_{n}$ by signed super-permutations:

$$
(i, i+1) \cdot\left(v_{1} \otimes \cdots \otimes v_{n}\right):=-(-1)^{\left|v_{i}\right|\left|v_{i+1}\right|} v_{1} \otimes \cdots v_{i} \otimes v_{i+1} \cdots \otimes v_{n} .
$$

- the second one (the internal grading) is obtained by assigning degree $i \in\{0,1\}$ to elements of $V_{1-i}$. Its degree $n$ homogeneous part is denoted by $\wedge(V)^{n}$, and we write $|x|$ for the internal degree of an element $x \in \wedge(V)$. In other words,

$$
\left|v_{1} \wedge \cdots \wedge v_{n}\right|=n-\sum_{i=1}^{n}\left|v_{i}\right| .
$$

Example 4.3. (a) If $V=V_{0}$ is purely even then $\wedge(V)=\wedge\left(V_{0}\right)$ is the ususal exterior algebra of $V_{0}$ and $\wedge^{n}(V)=\wedge^{n}\left(V_{0}\right)=\wedge(V)^{n}$.

(b) If $V=V_{1}$ is purely odd then $\wedge(V)=S\left(V_{1}\right)$ is the symmetric algebra of $V_{1}$. Moreover, $\wedge^{n}(V)=S^{n}\left(V_{1}\right)$ and $\wedge(V)$ is concentrated in degree 0 for the internal grading.

Observe that one has an isomorphism of bigraded vector spaces

$$
\begin{aligned}
S(\Pi V) & \stackrel{\sim}{\longmapsto} \wedge(V) \\
v_{1} \cdots v_{n} & \longmapsto(-1)^{\sum_{j=1}^{n}(j-1)\left|v_{j}\right|} v_{1} \wedge \cdots \wedge v_{n} .
\end{aligned}
$$

Remark that it remains true without the sign on the right. The motivation for this quite mysterious sign modification we make here is explained in the next paragraph.

Graded (super-)commutative algebras.

Definition 4.4. A graded algebra $A^{\bullet}$ is super-commutative if for any homogeneous elements $a, b$ one has $a \cdot b=(-1)^{|a||b|} b \cdot a$.

Example 4.5. (a) the symmetric algebra $S(V)$ of a super vector space is super-commutative with respect to its internal grading.

(b) the graded algebra $\Omega^{\bullet}(M)$ of differentiable forms on a smooth manifold $M$ is supercommutative.

The exterior algebra of a super vector space, with product $\wedge$ and the internal grading, is NOT a super-commutative algebra in general: for $v_{i} \in V_{i}(i=0,1)$ one has

$$
v_{0} \wedge v_{1}=-v_{1} \wedge v_{0} .
$$

One way to correct this drawback is to define a new product $\bullet$ on $\wedge(V)$ as follows: let $v \in \wedge^{k}(V)$ and $w \in \wedge^{l}(V)$ then

$$
v \bullet w:=(-1)^{k(|w|+l)} v \wedge w .
$$

In this situation one can check (this is an exercise) that the map (4.1) defines a graded algebra isomorphism

$$
(S(\Pi V), \cdot) \longrightarrow(\wedge(V), \bullet) .
$$

Graded Lie super-algebras.

Definition 4.6. A graded Lie super-algebra is a $\mathbb{Z}$-graded vector space $\mathfrak{g}^{\bullet}$ equipped with a degree zero graded linear map [,] $: \mathfrak{g} \otimes \mathfrak{g} \rightarrow \mathfrak{g}$ that is super-skew-symmetric, which means that

and satisfies the super-Jacobi identity

$$
[x, y]=-(-1)^{|x||y|}[y, x],
$$

$$
[x,[y, z]]=[[x, y], z]+(-1)^{|x||y|}[y,[x, z]] .
$$


Examples 4.7. (a) Let $A^{\bullet}$ be a graded associative algebra. Then $A$ equipped with the super-commutator

$$
[a, b]=a b-(-1)^{|a||b|} b a
$$

is a graded Lie super-algebra.

(b) Let $A^{\bullet}$ be a graded associative algebra and consider the space $\operatorname{Der}(A)$ of super derivations of $A$ : a degree $k$ graded linear map $d: A \rightarrow A$ is a super derivation if

$$
d(a b)=d(a) b+(-1)^{k|a|} a d(b) .
$$

$\operatorname{Der}(A)$ is stable under the super-commutator inside the graded associative algebra $\operatorname{End}(A)$ of (degree non-preserving) linear maps $A \rightarrow A$ (with product the composition).

The previous example motivates the following definition:

Definition 4.8. Let $\mathfrak{g}^{\bullet}$ be a graded Lie super-algebra.

1. A $\mathfrak{g}$-module is a graded vector space $V$ with a degree zero graded linear map $\mathfrak{g} \otimes V \rightarrow V$ such that

$$
x \cdot(y \cdot v)-(-1)^{|x||y|} y \cdot(x \cdot v)=[x, y] \cdot v .
$$

In other words it is a morphism $\mathfrak{g} \rightarrow \operatorname{End}(V)$ of graded Lie super-algebras.

2. If $V=A$ is a graded associative algebra, then one says that $\mathfrak{g}$ acts on $A$ by derivations if this morphism takes values in $\operatorname{Der}(A)$. In this case $A$ is called a $\mathfrak{g}$-module algebra.

\subsubsection{A remark on "graded" and "super".}

Throughout the text (and otherwise specified) graded always means $\mathbb{Z}$-graded and "super" stands for $\mathbb{Z} / 2 \mathbb{Z}$-graded. All our graded objects are obviously "super". Nevertheless "graded" and "super" do not play the same role; namely, in all definitions structures (e.g. a product) are graded and properties (e.g. the commutativity) are "super" (it has some importance only in the case there is an action of the symmetric group).

For example, a graded Lie super algebra is NOT a graded Lie algebra in the usual sens: $\operatorname{End}(V)$ with the usual commutator is a graded Lie agebra while it is a Lie super-algebra with the super-commutator.

\subsection{Hochschild cohomology strikes back.}

Hochschild cohomology of a graded algebra.

Let $A^{\bullet}$ be a graded associative algebra. Its Hochschild complex $C^{\bullet}(A, A)$ is defined as the sum of spaces of (not necessarily graded) linear maps $A^{\otimes n} \rightarrow A$. Let us denote by $|\cdot|$ the degree of those linear maps; the grading on $C^{\bullet}(A, A)$ is given by the total degree, denoted $\|\cdot\|$. For any $f: A^{\otimes m} \rightarrow A,\|f\|=|f|+m$. The differential $d_{H}$ is given by

$$
d_{H}(f)\left(a_{0}, \ldots, a_{m}\right)=(-1)^{|f|\left|a_{0}\right|} a_{0} f\left(a_{1}, \ldots, a_{m}\right)+\sum_{i=1}^{m}(-1)^{i} f\left(a_{0}, \ldots, a_{i-1} a_{i}, \ldots, a_{m}\right)
$$

Again it is easy to prove that $d_{H} \circ d_{H}=0$.

As in paragraph $2.1\left(C^{\bullet}(A, A), d_{H}\right)$ is a DG algebra with product $\cup$ defined by

$$
f \cup g\left(a_{1}, \ldots, a_{m+n}\right):=(-1)^{|g|\left(\left|a_{1}\right|+\cdots+\left|a_{m}\right|\right)} f\left(a_{1}, \ldots, a_{m}\right) g\left(a_{m+1}, \ldots, a_{m+n}\right) .
$$

Hochschild cohomology of a DG algebra.

Let $A^{\bullet}$ be a graded associative algebra. We now prove that $C^{\bullet}(A, A)$ is naturally a $\operatorname{Der}(A)$-module.

For any $d \in \operatorname{Der}(A)$ and any $f \in C^{\bullet}(A, A)$ one defines

$$
d(f)\left(a_{1}, \ldots, a_{m}\right):=d\left(f\left(a_{1}, \ldots, a_{m}\right)\right)-(-1)^{|d|(\|f\|-1)} \sum_{i=1}^{m}(-1)^{(i-1)(m-1)} f\left(a_{1}, \ldots, d a_{i}, \ldots, a_{m}\right) .
$$


In other words, $d$ is defined as the unique degree $|d|$ derivation for the cup product that is given by the super-commutator on linear maps $A \rightarrow A$.

Moreover, one can easily check that $d \circ d_{H}+d_{H} \circ d=0$.

Therefore if $\left(A^{\bullet}, d\right)$ is a DG algebra then its Hochschild complex is $C^{\bullet}(A, A)$ together with $d_{H}+d$ as a differential. It is again a DG algebra, and we denote its cohomology by $H H^{\bullet}(A, d)$.

Remark 4.9 (Deformation theoretic interpretation). In the spirit of the discussion in paragraph 2.1 one can prove that $H H^{2}(A, d)$ is the set of equivalence classes of infinitesimal deformations of $A$ as an $A_{\infty}$-algebra (an algebraic structure introduced by J. Stasheff in [31]) and that the obstruction to extending such deformations order by order lies in $H H^{3}(A, d)$.

More generally, if $\left(M^{\bullet}, d_{M}\right)$ is a DG bimodule over $\left(A^{\bullet}, d_{A}\right)$ then the Hochschild complex $C^{\bullet}(A, M)$ of $A$ with values in $M$ consists of linear maps $A^{\otimes n} \rightarrow M(n \geq 0)$ and the differential is $d_{H}+d$, with $d_{H}$ given by (4.2) and

$d(f)\left(a_{1}, \ldots, a_{m}\right):=d_{M}\left(f\left(a_{1}, \ldots, a_{m}\right)\right)-(-1)^{|d|(\|f\|-1)} \sum_{i=1}^{m}(-1)^{(i-1)(m-1)} f\left(a_{1}, \ldots, d_{A} a_{i}, \ldots, a_{m}\right)$.

Hohschild cohomology of the Chevalley algebra.

One has the following important result:

Theorem 4.10. Let $\mathfrak{g}$ be a finite dimensional Lie algebra. Then there is an isomorphism of graded algebras $H H^{\bullet}\left(\wedge \mathfrak{g}^{*}, d_{C}\right) \stackrel{\sim}{\longrightarrow} H H^{\bullet}(U \mathfrak{g})$.

Let us emphazise that this result is related to some general considerations about Koszul duality for quadratic algebras (see e.g. [28]).

Proof. Thanks to Theorem 2.5 it is sufficient to prove that $H H^{\bullet}\left(\wedge \mathfrak{g}^{*}, d_{C}\right) \stackrel{\sim}{\longrightarrow} H^{\bullet}(\mathfrak{g}, U \mathfrak{g})$. Let us define a linear map

$$
C\left(\wedge \mathfrak{g}^{*}, \wedge \mathfrak{g}^{*}\right)=\wedge \mathfrak{g}^{*} \otimes T(\wedge \mathfrak{g}) \longrightarrow \wedge \mathfrak{g}^{*} \otimes U(\mathfrak{g})=C(\mathfrak{g}, U \mathfrak{g})
$$

given by the projection $p: T(\wedge \mathfrak{g}) \rightarrow T(\mathfrak{g}) \rightarrow U(\mathfrak{g})$. It is an exercise to verify that it defines a morphism of DG algebras

$$
\left(C\left(\wedge \mathfrak{g}^{*}, \wedge \mathfrak{g}^{*}\right), d_{H}+d_{C}\right) \longrightarrow\left(C(\mathfrak{g}, U \mathfrak{g}), d_{C}\right) .
$$

It remains to prove that it is a quasi-ismorphism. We use a spectral sequence argument.

Lemma 4.11. We equip $k$ (with the zeroe differential) with the $\left(\wedge \mathfrak{g}^{*}, d_{C}\right)$-DG-bimodule structure given by the projection $\epsilon: \wedge \mathfrak{g}^{*} \rightarrow k$ (left and right actions coincide). Then $H^{\bullet}\left(\left(\wedge \mathfrak{g}^{*}, d_{C}\right), k\right) \cong U(\mathfrak{g})$.

Proof of the lemma. We consider the following filtration on $C^{\bullet}\left(\left(\wedge \mathfrak{g}^{*}, d_{C}\right), k\right): F^{p} C^{n}\left(\left(\wedge \mathfrak{g}^{*}, d_{C}\right), k\right)$ is given by linear forms on

$$
\bigoplus_{\substack{k \geq 0 \\ i_{1}+\cdots+i_{k}=k-n}} \wedge^{i_{1}}\left(\mathfrak{g}^{*}\right) \otimes \cdots \otimes \wedge^{i_{k}}\left(\mathfrak{g}^{*}\right)
$$

that vanish on the components for which $n-k<p$. Then we have

$$
E_{0}^{p, q}=\operatorname{Lin}\left(\bigoplus_{i_{1}+\cdots+i_{q}=-p} \wedge^{i_{1}}\left(\mathfrak{g}^{*}\right) \otimes \cdots \otimes \wedge^{i_{q}}\left(\mathfrak{g}^{*}\right), k\right) \quad \text { with } \quad d_{0}=d_{H} .
$$

Applying a "super" version of Lemma 2.6 to $V=\Pi\left(\mathfrak{g}^{*}\right)$ one obtains that

$$
E_{1}^{p, q}=E_{1}^{-q, q}=\wedge^{q}\left(\Pi\left(\mathfrak{g}^{*}\right)^{*}\right)=S^{q}(\mathfrak{g}),
$$


and that the spectral sequence stabilizes at $E_{1}$. Consequently $\operatorname{Gr}\left(H^{\bullet}\left(\left(\wedge \mathfrak{g}^{*}, d_{C}\right), k\right)\right) \cong$ $S(\mathfrak{g})=\operatorname{Gr}(U(\mathfrak{g}))$ and the isomorphism is given by the following composed map

$$
T(\wedge(\mathfrak{g})) \longrightarrow T(\mathfrak{g}) \longrightarrow S(\mathfrak{g}) .
$$

This ends the proof of the lemma.

Lemma 4.12. The map (4.3) is a quasi-isomorphism: $H H^{\bullet}\left(\wedge \mathfrak{g}^{*}, d_{C}\right) \cong H^{\bullet}(\mathfrak{g}, U \mathfrak{g})$.

Proof of the lemma. Let us consider the descending filtration on the Hochschild complex that is induced from the following descending filtration on $\wedge \mathfrak{g}^{*}$ :

$$
F^{n}\left(\wedge \mathfrak{g}^{*}\right):=\bigoplus_{k \geq n} \wedge^{k} \mathfrak{g}^{*} .
$$

Then the zeroth term of the associated spectral sequence (of algebras) is

$$
E_{0}^{\bullet \bullet \bullet}=\wedge^{\bullet} \mathfrak{g}^{*} \otimes C^{\bullet}\left(\left(\wedge \mathfrak{g}^{*}, d_{C}\right), k\right) \quad \text { with } \quad d_{0}=\mathrm{id} \otimes\left(d_{H}+d_{C}\right) .
$$

Then using Lemma 4.11 one obtains that $E_{1}^{\bullet, \bullet}=E_{1}^{\bullet, 0}=\wedge^{\bullet} \mathfrak{g}^{*} \otimes U \mathfrak{g}$ with $d_{1}=d_{C}$. Therefore the spectral sequence stabilizes at $E_{2}$ and the result follows.

This ends the proof of the Theorem. 


\section{The Duflo-Kontsevich isomorphism for $Q$-SPaCeS}

In this section we prove a general Duflo type result for $Q$-spaces, i.e. superspaces equipped with a square zero degree one vector field. This result implies in particular the cohomological version of the Duflo theorem 1.11, and will be used in the sequel to prove the Kontsevich theorem 3.5. This approach makes more transparent the analogy between the adjoint action and the Atiyah class.

\subsection{Statement of the result.}

Let $V$ be a superspace.

Hochschild-Kostant-Rosenberg for superspaces.

We introduce

- $\mathcal{O}_{V}:=S\left(V^{*}\right)$, the graded super-commutative algebra of functions on $V$;

- $\mathfrak{X}_{V}:=\operatorname{Der}\left(\mathcal{O}_{V}\right)=S\left(V^{*}\right) \otimes V$, the graded Lie super-algebra of vector fields on $V$;

- $T_{\text {poly }} V:=S\left(V^{*} \oplus \Pi V\right) \cong \wedge_{\mathcal{O}_{V}} \mathfrak{X}_{V}$, the $\mathfrak{X}_{V}$-module algebra of polyvector fields on $V$.

We now describe the gradings we will consider.

The grading on $\mathcal{O}_{V}$ is the internal one: elements in $V_{i}^{*}$ have degree $i$.

The grading on $\mathfrak{X}_{V}$ is the restriction of the natural grading on $\operatorname{End}\left(\mathcal{O}_{V}\right)$ : elements in $V_{i}^{*}$ have degree $i$ and elements in $V_{i}$ have degree $-i$.

There are three different gradings on $T_{\text {poly }} V$ :

(i) the one given by the number of arguments: degree $k$ elements lie in $\wedge_{\mathcal{O}_{V}}^{k} \mathfrak{X}_{V}$. In other words elements in $V^{*}$ have degree 0 and elements in $V$ have degree 1 ;

(ii) the one induced by $\mathfrak{X}_{V}$ : elements in $V_{i}^{*}$ have degree $i$ and elements in $V_{i}$ have degree $-i$. It is denoted by $|\cdot|$;

(iii) the total (or internal) degree: it is the sum of the previous ones. Elements in $V_{i}^{*}$ have degree $i$ and elements in $V_{i}$ have degree $1-i$. It is denoted by $\|\cdot\|$.

Unless otherwise precised, we always consider the total grading on $T_{\text {poly }} V$ in the sequel.

We also have

- the $\mathfrak{X}_{V}$-module algebra $D_{V}$ of differential operators on $V$, which is the subalgebra of $\operatorname{End}\left(\mathcal{O}_{V}\right)$ generated by $\mathcal{O}_{V}$ and $\mathfrak{X}_{V}$;

- the $\mathfrak{X}_{V}$-module algebra $D_{\text {poly }} V$ of polydifferential operators on $V$, which consists of multilinear maps $\mathcal{O}_{V} \otimes \cdots \otimes \mathcal{O}_{V} \rightarrow \mathcal{O}_{V}$ being differential operators in each argument.

The grading on $D_{V}$ is the restriction of the natural grading on $\operatorname{End}\left(\mathcal{O}_{V}\right)$. As for $T_{\text {poly }}$ there are three different gradings on $D_{\text {poly }}$ : the one given by the number of arguments, the one induced by $D_{V}($ denoted $|\cdot|)$, and the one given by their sum (denoted $\left.\|\cdot\|\right)$. $D_{\text {poly }}$ is then a subcomblex of the Hochschild complex of the algebra $\mathcal{O}_{V}$ introduced in the previous Section, since it is obviously preserved by the differential $d_{H}$.

An appropriate super-version of Lemma 2.6 gives the following result:

Proposition 5.1. The natural inclusion $I_{H K R}:\left(T_{\text {poly }} V, 0\right) \hookrightarrow\left(D_{\text {poly }} V, d_{H}\right)$ is a quasiisomorphism of complexes, that induces an isomorphism of algebras in cohomology.

Cohomological vector fields.

Definition 5.2. A cohomological vector field on $V$ is a degree one vector field $Q \in \mathfrak{X}_{V}$ that is integrable: $[Q, Q]=2 Q \circ Q=0$. A superspace equipped with a cohomological vector field is called a $Q$-space.

Let $Q$ be a cohomological vector field on $V$. Then $\left(T_{\text {poly }} V, Q \cdot\right)$ and $\left(D_{\text {poly }} V, d_{H}+Q \cdot\right)$ are DG algebras. By a spectral sequence argument one can show that $I_{H K R}$ still defines a quasi-isomorphism of complexes between them. Nevertheless it does no longer respect the product on the level of cohomology. Similarly to theorems 1.11 and 3.5, Theorem 5.3 below remedy to this situation. 
Let us remind the reader that the graded algebra of differential forms on $V$ is $\Omega(V):=$ $S\left(V^{*} \oplus \Pi V^{*}\right)$ and that it is equipped with the following structures:

- for any element $x \in V^{*}$ we write $\mathrm{d} x$ for the corresponding element in $\Pi V^{*}$, and then we define a differential on $\Omega(V)$, the de Rham differential, given on generators by $\mathrm{d}(x)=\mathrm{d} x$ and $\mathrm{d}(\mathrm{d} x)=0$

- there is an action $\iota$ of differential forms on polyvector fields by contraction, where $x \in V^{*}$ acts by left multiplication and $\mathrm{d} x$ acts by derivation in the following way: for any $y \in V^{*}$ and $v \in \Pi V$ one has

$$
\iota_{\mathrm{d} x}(y)=0 \quad \text { and } \quad \iota_{\mathrm{d} x}(v)=\langle x, v\rangle .
$$

We then define the (super)matrix valued one-form $\Xi \in \Omega^{1}(V) \otimes \operatorname{End}(V[1])$ with coefficients explicitly given by

$$
\Xi_{i}^{j}:=\mathrm{d}\left(\frac{\partial Q^{j}}{\partial x^{i}}\right)=\frac{\partial^{2} Q^{j}}{\partial x^{k} \partial x^{i}} \mathrm{~d} x^{k},
$$

where $x^{1}, \ldots, x^{n}$ is a basis of coordinates on $V$. Observe that it does not depend on the choice of coordinates, and set

$$
j(\Xi):=\operatorname{Ber}\left(\frac{1-e^{-\Xi}}{\Xi}\right) \in \Omega(V) .
$$

Theorem 5.3. $I_{H K R} \circ \iota_{j(\Xi)^{1 / 2}}:\left(T_{\text {poly }} V, Q \cdot\right) \longrightarrow\left(D_{\text {poly }} V, d_{H}+Q \cdot\right)$ defines a quasiisomorphism of complexes that induces an algebra isomorphism on cohomology.

As for Theorems 1.2, 1.11 and 3.5 one can replace $j(\Xi)$ by

$$
\widetilde{j}(\Xi):=\operatorname{Ber}\left(\frac{e^{\Xi / 2}-e^{-\Xi / 2}}{\Xi}\right) .
$$

\subsection{Application: proof of the Duflo Theorem.}

In this paragraph we discuss an important application of Theorem 5.3, namely the "classical" Theorem of Duflo (see Theorem 1.2 and 1.11): before entering into the details of the proof, we need to establish a correspondence between the algebraic tools of Duflo's Theorem and the differential-geometric objects of 5.3 .

We consider a finite dimensional Lie algebra $\mathfrak{g}$, to which we associate the superspace $V=\Pi \mathfrak{g}$. In this setting, we have the following identification:

$$
\mathcal{O}_{V} \cong \wedge^{\bullet} \mathfrak{g}^{*},
$$

i.e. the superalgebra of polynomial functions on $V$ is identified with the graded vector space defining the Chevalley-Eilenberg graded algebra for $\mathfrak{g}$ with values in the trivial $\mathfrak{g}$-module; we observe that the natural grading of the Chevalley-Eilenberg complex of $\mathfrak{g}$ corresponds to the aforementioned grading of $\mathcal{O}_{V}$. The Chevalley-Eilenberg differential $d_{C}$ identifies, under the above isomorphism, with a vector field $Q$ of degree 1 on $V ; Q$ is cohomological, since $d_{C}$ squares to 0 .

In order to make things more understandable, we make some explicit computations w.r.t. supercoordinates on $V$. For this purpose, a basis $\left\{e_{i}\right\}$ of $\mathfrak{g}$ determines a system of (purely odd) coordinates $\left\{x^{i}\right\}$ on $V$ : the previous identification can be expressed in terms of these coordinates as

$$
x^{i_{1}} \cdots x^{i_{p}} \mapsto \varepsilon^{i_{1}} \wedge \cdots \wedge \varepsilon^{i_{p}}, 1 \leq i_{1}<\cdots<i_{p} \leq n,
$$

$\left\{\varepsilon^{i}\right\}$ being the dual basis of $\left\{e_{i}\right\}$. Hence, w.r.t. these odd coordinates, $Q$ can be written as

$$
Q=-\frac{1}{2} c_{j k}^{i} x^{j} x^{k} \frac{\partial}{\partial x^{i}},
$$

where $c_{j k}^{i}$ are the structure constants of $\mathfrak{g}$ w.r.t. the basis $\left\{e_{i}\right\}$. It is clear that $Q$ has degree 1 and total degree 2 . 
Lemma 5.4. The $D G$ algebra $\left(T_{\text {poly }} V, Q \cdot\right)$ identifies naturally with the Chevalley-Eilenberg $D G$ algebra $\left(C^{\bullet}(\mathfrak{g}, S(\mathfrak{g})), d_{C}\right)$ associated to the $\mathfrak{g}$-module algebra $S(\mathfrak{g})$.

Proof. By the very definition of $V$, we have an isomorphism of graded algebras

$$
S\left(V^{*} \oplus \Pi V\right) \cong \wedge^{\bullet}\left(\mathfrak{g}^{*}\right) \otimes S(\mathfrak{g}) .
$$

More explicitly, in terms of the aforementioned supercoordinates, the previous isomorphism is given by

$$
x^{i_{1}} \cdots x^{i_{p}} \partial_{x^{j_{1}}} \wedge \cdots \wedge \partial_{x^{j_{q}}} \mapsto \varepsilon^{i_{1}} \wedge \cdots \wedge \varepsilon^{i_{p}} \otimes e_{j_{1}} \cdots e_{j_{q}},
$$

where the indices $\left(i_{1}, \ldots, i_{p}\right)$ form a strictly increasing sequence.

It remains to prove that the action of $Q$ on $T_{\text {poly }} V$ coincides, under the previous isomorphism, with the Chevalley-Eilenberg differential $d_{C}$ on $\wedge^{\bullet}\left(\mathfrak{g}^{*}\right) \otimes S(\mathfrak{g})$. It suffices to prove the claim on generators, i.e. on the coordinates functions $\left\{x^{i}\right\}$ and on the derivations $\left\{\partial_{x^{i}}\right\}$ : the action of $Q$ on both of them is given by

$$
\begin{aligned}
Q \cdot x^{i} & =Q\left(x^{i}\right)=-\frac{1}{2} c_{j k}^{i} x^{j} x^{k}, \\
Q \cdot \partial_{x^{i}} & =\left[Q, \partial_{x^{i}}\right]=-c_{i j}^{k} x^{j} \partial_{x^{k}} .
\end{aligned}
$$

Under the above identification between $T_{\text {poly }} V$ and $\wedge^{\bullet}\left(\mathfrak{g}^{*}\right) \otimes S(\mathfrak{g})$, it is clear that $Q$ identifies with $d_{C}$, thus the claim follows.

Similar arguments and computations imply the following

Lemma 5.5. There is a natural isomorphism from the $D G$ algebra $\left(D_{\text {poly }} V, d_{H}+Q \cdot\right)$ to the DG algebra $\left(C^{\bullet}\left(\wedge \mathfrak{g}^{*}, \wedge \mathfrak{g}^{*}\right), d_{H}+d_{C}\right)$.

Coupling these results with Lemma 4.12, we obtain the following commutative diagram of quasi-isomorphisms of complexes, all inducing algebra isomorphisms on the level of cohomology:

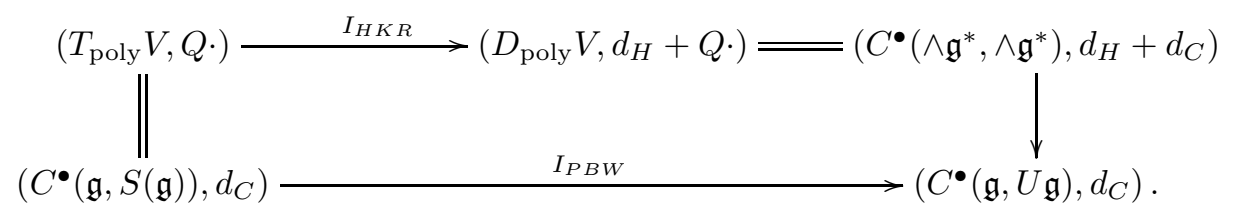

Using the previously computed explicit expression for the cohomological vector field $Q$ on $V$, one can easily prove the following

Lemma 5.6. Under the obvious identification $V[1] \cong \mathfrak{g}$, the supermatrix valued 1-form $\Xi$, restricted to $\mathfrak{g}$, which we implicitly identify with the space of vector fields on $V$ with constant coefficients, satisfies

$$
\Xi=\mathrm{ad}
$$

Proof. Namely, since

we have

$$
Q=-\frac{1}{2} c_{j k}^{i} x^{j} x^{k} \partial_{x^{i}}
$$

$$
\Xi_{j}^{i}=\mathrm{d}\left(\partial_{x^{j}} Q^{i}\right)=-c_{j k}^{i} \mathrm{~d} x^{k}=c_{k j}^{i} \mathrm{~d} x^{k},
$$

and the claim follows by a direct computation, when e.g. evaluating $\Xi$ on $e_{k}=\partial_{x^{k}}$.

Hence, Theorem 5.3, together with Lemma 5.4, 5.5 and 5.6 implies Theorem 1.11. QED.

\subsection{Strategy of the proof.}

The proof of Theorem 5.3 occupies the next three sections. In this paragraph we explain the strategy we are going to adopt in Sections 6, 7, 8 and 9. 
The homotopy argument.

Our approach relies on a homotopy argument (in the context of deformation quantization, this argument is sketch by Kontsevich in [22] and detailed by Manchon and Torossian in [26]). Namely, we construct a quasi-isomorphism of complexes ${ }^{5}$

$$
\mathcal{U}_{Q}:\left(T_{\text {poly }} V, Q \cdot\right) \longrightarrow\left(D_{\text {poly }} V, d_{H}+Q \cdot\right)
$$

and a degree -1 map

$$
\mathcal{H}_{Q}: T_{\text {poly }} V \otimes T_{\text {poly }} V \longrightarrow D_{\text {poly }} V
$$

satisfying the homotopy equation

$$
\begin{aligned}
& \mathcal{U}_{Q}(\alpha) \cup \mathcal{U}_{Q}(\beta)-\mathcal{U}_{Q}(\alpha \wedge \beta)= \\
& =\left(\mathrm{d}_{H}+Q \cdot\right) \mathcal{H}_{Q}(\alpha, \beta)+\mathcal{H}_{Q}(Q \cdot \alpha, \beta)+(-1)^{\|\alpha\|} \mathcal{H}_{Q}(\alpha, Q \cdot \beta)
\end{aligned}
$$

for any polyvector fields $\alpha, \beta \in T_{\text {poly }} V$.

We sketch below the construction of $\mathcal{U}_{Q}$ and $\mathcal{H}_{Q}$.

Formulae for $\mathcal{U}_{Q}$ and $\mathcal{H}_{Q}$, and the scheme of the proof.

For any polyvector fields $\alpha, \beta \in T_{\text {poly }} V$ and functions $f_{1}, \ldots, f_{m}$ we set

$$
\mathcal{U}_{Q}(\alpha)\left(f_{1}, \ldots, f_{m}\right):=\sum_{n \geq 0} \frac{\hbar^{n}}{n !} \sum_{\Gamma \in \mathcal{G}_{n+1, m}} \mathcal{W}_{\Gamma} B_{\Gamma}(\alpha, \underbrace{Q, \ldots, Q}_{n \text { times }})\left(f_{1}, \ldots, f_{m}\right)
$$

and

$$
\mathcal{H}_{Q}(\alpha, \beta)\left(f_{1}, \ldots, f_{m}\right):=\sum_{n \geq 0} \frac{\hbar^{n}}{n !} \sum_{\Gamma \in \mathcal{G}_{n+2, m}} \widetilde{\mathcal{W}}_{\Gamma} B_{\Gamma}(\alpha, \beta, \underbrace{Q, \ldots, Q}_{n \text { times }})\left(f_{1}, \ldots, f_{m}\right) .
$$

The sets $\mathcal{G}_{n, m}$ are described by suitable directed graphs with two types of vertices, the "weights" $\mathcal{W}_{\Gamma}$ and $\widetilde{\mathcal{W}}_{\Gamma}$ are scalar associated to such graphs, and $B_{\Gamma}$ are polydifferential operators associated to those graphs.

We define in the next paragraph the sets $\mathcal{G}_{n, m}$ and the associated polydifferential operators $B_{\Gamma}$. The weights $\mathcal{W}_{\Gamma}$ and $\widetilde{\mathcal{W}}_{\Gamma}$ are introduced in Section 6 and 8 , respectively. In Section 7 (resp. 8) we prove that $\mathcal{U}(\alpha \wedge \beta)$ and $\mathcal{U}(\alpha) \cup \mathcal{U}(\beta)$ (resp. the r.h.s. of (5.1)) are given by a formula similar to $(5.3)$ with new weights $\mathcal{W}_{\Gamma}^{0}$ and $\mathcal{W}_{\Gamma}^{1}\left(\right.$ resp. $\left.-\mathcal{W}_{\Gamma}^{2}\right)$, so that, in fine, the homotopy property $(5.1)$ reduces to

$$
\mathcal{W}_{\Gamma}^{0}=\mathcal{W}_{\Gamma}^{1}+\mathcal{W}_{\Gamma}^{2}
$$

Polydifferential operators associated to a graph.

Let us consider, for given positive integers $n$ and $m$, the set $\mathcal{G}_{n, m}$ of directed graphs described as follows:

(1) there are $n$ vertices of the "first type", labeled by $1, \ldots, n$;

(2) there are $m$ vertices of the "second type", labeled by $\overline{1}, \ldots, \bar{m}$;

(3) the vertices of the second type have no outgoing edge;

(4) there are no loop (a loop is an edge having the same source and target) and no double edge (a double edge is a pair of edges with common source and common target);

Let us define $\tau=\operatorname{id}_{V_{0}}-\operatorname{id}_{V_{1}} \in V^{*} \otimes V$, and let it acts as a derivation on $S(\Pi V) \otimes S\left(V^{*}\right)$ simply by contraction. In other words, using coordinates $\left(x^{i}\right)_{i}$ on $V$ and dual odd coordinates $\left(\theta_{i}\right)_{i}$ on $\Pi V^{*}$ one has

$$
\tau=\sum_{i}(-1)^{\left|x^{i}\right|} \partial_{\theta_{i}} \otimes \partial_{x^{i}}
$$

This action naturally extends to $S\left(V^{*} \oplus \Pi V\right) \otimes S\left(V^{*} \oplus \Pi V\right)$ (the action on additional variables is zero). For any finite set $I$ and any pair $(i, j)$ of distinct elements in $I$ we denote by $\tau_{i j}$ the

\footnotetext{
${ }^{5}$ It is the first structure map of Kontsevich's tangent $L_{\infty}$-quasi-isomorphism [22].
} 
endomorphism of $S\left(V^{*} \oplus \Pi V\right)^{\otimes I}$ given by $\tau$ which acts by the identity on the $k$-th factor for any $k \neq i, j$.

Let us then chose a graph $\Gamma \in \mathcal{G}_{n, m}$, polyvector fields $\gamma_{1}, \ldots, \gamma_{n} \in T_{\text {poly }} V=S\left(V^{*} \oplus \Pi V\right)$, and functions $f_{1}, \ldots, f_{m} \in \mathcal{O}_{V} \subset S\left(V^{*} \oplus \Pi V\right)$. We define

$$
B_{\Gamma}\left(\gamma_{1}, \ldots, \gamma_{n}\right)\left(f_{1}, \ldots, f_{m}\right):=\epsilon\left(\mu\left(\prod_{(i, j) \in \mathcal{E}(\Gamma)} \tau_{i j}\left(\gamma_{1} \otimes \cdots \otimes \gamma_{n} \otimes f_{1} \otimes \cdots \otimes f_{m}\right)\right)\right),
$$

where $\mathcal{E}(\Gamma)$ denotes the set of edges of the graph $\Gamma, \mu: S\left(V^{*} \oplus \Pi V\right)^{\otimes(n+m)} \rightarrow S\left(V^{*} \oplus \Pi V\right)$ is the product, and $\epsilon: S\left(V^{*} \oplus \Pi V\right) \rightarrow S\left(V^{*}\right)=\mathcal{O}_{V}$ is the projection onto 0-polyvetcor fields (defined by $\theta_{i} \mapsto 0$ ).

Remark 5.7. (a) If the number of outgoing edges of a first type vertex $i$ differs from $\left|\gamma_{i}\right|$ then the r.h.s. of(5.4) is obviously zero.

(b) We could have allowed edges outgoing from a second type vertex, but in this case the r.h.s. of (5.4) is obviously zero.

(c) There is an ambiguity in the order of the product of endomorphisms $\tau_{i j}$. Since each $\tau_{i j}$ has degree one then there is a sign ambiguity in the r.h.s. of (5.4). Fortunately the same ambiguity appears in the definition of the weights $\mathcal{W}_{\Gamma}$ and $\widetilde{\mathcal{W}}_{\Gamma}$, insuring us that the expression (5.2) and (5.3) for $\mathcal{U}_{Q}$ and $\mathcal{H}_{Q}$ are well-defined.

Example 5.8. Consider three polyvector fields $\gamma_{1}=\gamma_{1}^{i j k} \theta_{i} \theta_{j} \theta_{k}, \gamma_{2}=\gamma_{2}^{l p} \theta_{l} \theta_{p}$ and $\gamma_{3}=$ $\gamma_{3}^{q r} \theta_{q} \theta_{r}$, and functions $f_{1}, f_{2} \in \mathcal{O}_{V}$. If $\Gamma \in \mathcal{G}_{3,2}$ is given by the Figure 1 then

$$
B_{\Gamma}\left(\gamma_{1}, \gamma_{2}, \gamma_{3}\right)\left(f_{1}, f_{2}\right)= \pm \gamma_{1}^{i j k}\left(\partial_{i} \partial_{q} \gamma_{2}^{l p}\right)\left(\partial_{j} \gamma_{3}^{q r}\right)\left(\partial_{l} f_{1}\right)\left(\partial_{r} \partial_{p} \partial_{k} f_{2}\right)
$$

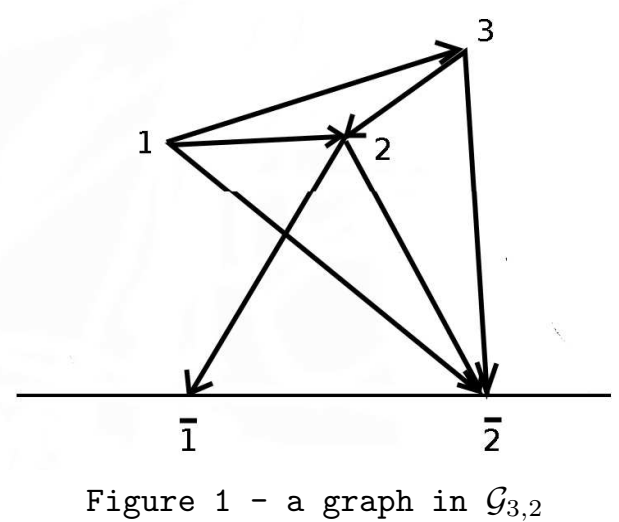




\section{Configuration spaces And integral Weights}

The main goal of this section is to define the weights $\mathcal{W}_{\Gamma}$ appearing in the defining formula (5.2) for $\mathcal{U}_{Q}$. These weights are defined as integrals over suitable configuration spaces of points in the upper half-plane. We therefore introduce these configuration spaces, and also their compactifications $\grave{a}$ la Fulton-MacPherson, which insure us that the integral weights truly exists. Furthermore, the algebraic identities illustrated in Sections 7 and 8 follow from factorization properties of these integrals, which in turn rely on Stokes' Theorem: thus, we discuss the boundary of the compactified configuration spaces.

\subsection{The configuration spaces $C_{n, m}^{+}$.}

We denote by $\mathcal{H}$ the complex upper half-plane, i.e. the set of all complex numbers, whose imaginary part is strictly bigger than 0 ; further, $\mathbb{R}$ denotes here the real line in the complex plane.

Definition 6.1. For any two positive integers $n, m$, we denote by Conf $_{n, m}^{+}$the configuration space of $n$ points in $\mathcal{H}$ and $m$ points in $\mathbb{R}$, i.e. the set of $n+m$-tuples

$$
\left(z_{1}, \ldots, z_{n}, q_{1}, \ldots, q_{m}\right) \in \mathcal{H}^{n} \times \mathbb{R}^{m},
$$

satisfying $z_{i} \neq z_{j}$ if $i \neq j$ and $q_{1}<\cdots<q_{m}$.

It is clear that Conf $_{n, m}^{+}$is a real manifold of dimension $2 n+m$.

We consider further the semidirect product $G_{2}:=\mathbb{R}^{+} \ltimes \mathbb{R}$, where $\mathbb{R}^{+}$acts on $\mathbb{R}$ by rescaling: it is a Lie group of real dimension 2. The group $G_{2}$ acts on Conf ${ }_{n, m}^{+}$by translations and homotheties simultaneously on all components, by the explicit formula

$$
\left((a, b),\left(z_{1}, \ldots, z_{n}, q_{1}, \ldots, q_{m}\right)\right) \longmapsto\left(a z_{1}+b, \ldots, a z_{n}+b, a q_{1}+b, \ldots, a q_{m}+b\right),
$$

for any pair $(a, b)$ in $G_{2}$. It is easy to verify that $G_{2}$ preserves Conf $_{n, m}^{+}$; easy computations also show that $G_{2}$ acts freely on Conf $_{n, m}^{+}$precisely when $2 n+m \geq 2$. In this case, we may take the quotient space $\operatorname{Conf}_{n, m}^{+} / G_{2}$, which will be denoted by $C_{n, m}^{+}$: in fact, we will refer to it, rather than to $\mathrm{Conf}_{n, m}^{+}$, as to the configuration space of $n$ points in $\mathcal{H}$ and $m$ points in $\mathbb{R}$. It is also a real manifold of dimension $2 n+m-2$.

Remark 6.2. We will not be too much concerned about orientations of configuration spaces; anyway, it is still useful to point out that $C_{n, m}^{+}$is an orientable manifold. In fact, Conf ${ }_{n, m}^{+}$ is an orientable manifold, as it possesses a natural volume form,

$$
\Omega:=d x_{1} \wedge d y_{1} \wedge \cdots \wedge d x_{n} \wedge d y_{n} \wedge d q_{1} \wedge \cdots d q_{m},
$$

using real coordinates $z=x+\mathrm{i} y$ for a point in $\mathcal{H}$. The volume form $\Omega$ descends to a volume form on $C_{n, m}^{+}$: this is a priori not so clear. In fact, the idea is to use the action of $G_{2}$ on Conf $_{n, m}^{+}$to choose certain preferred representatives for elements of $C_{n, m}^{+}$, which involve spaces of the form $\mathrm{Conf}_{n_{1}, m_{1}}^{+}$, for different choices of $n_{1}$ and $m_{1}$. The orientability of Conf $n_{n_{1}, m_{1}}^{+}$implies the orientability of $C_{n, m}^{+}$; we refer to [3] for a careful explanation of choices of representatives for $C_{n, m}^{+}$and respective orientation forms.

We also need to introduce another kind of configuration space.

Definition 6.3. For a positive integer $n$, we denote by $\operatorname{Conf}_{n}$ the configuration space of $n$ points in the complex plane, i.e. the set of all $n$-tuples of points in $\mathbb{C}$, such that $z_{i} \neq z_{j}$ if $i \neq j$.

It is a complex manifold of complex dimension $n$, or also a real manifold of dimension $2 n$.

We consider the semidirect product $G_{3}=\mathbb{R}^{+} \ltimes \mathbb{C}$, which is a real Lie group of dimension 3 ; it acts on $\operatorname{Conf}_{n}$ by the following rule:

$$
\left((a, b),\left(z_{1}, \ldots, z_{n}\right)\right) \longmapsto\left(a z_{1}+b, \ldots, a z_{n}+b\right) .
$$


The action of $G_{3}$ on Conf $_{n}$ is free, precisely when $n \geq 2$ : in this case, we define the (open) configuration space $C_{n}$ of $n$ points in the complex plane as the quotient space $\operatorname{Conf}_{n} / G_{3}$, and it can be proved that $C_{n}$ is a real manifold of dimension $2 n-3$. Following the same patterns in Remark 6.2 , one can show that $C_{n}$ is an orientable manifold.

\subsection{Compactification of $C_{n}$ and $C_{n, m}^{+}$à la Fulton-MacPherson.}

In order to clarify forthcoming computations in Section 8, we need certain integrals over the configuration spaces $C_{n, m}^{+}$and $C_{n}$ : these integrals are a priori not well-defined, and we have to show that they truly exist. Later, we make use of Stokes' Theorem on these integrals to deduce the relevant algebraic properties of $\mathcal{U}_{Q}$ : therefore we will need the boundary contributions to the aforementioned integrals. Kontsevich [22] introduced for this purpose nice compactifications $\bar{C}_{n, m}^{+}$of $C_{n, m}^{+}$which solve, on the one hand, the problem of the existence of such integrals (their integrand extend smoothly to $\bar{C}_{n, m}^{+}$, and so they can be understood as integrals of smooth forms over compact manifolds); on the other hand, the boundary stratifications of $\bar{C}_{n, m}^{+}$and $\bar{C}_{n}$ and their combinatorics yield the desired aforementioned algebraic properties.

\section{Definition and examples.}

The main idea behind the construction of $\bar{C}_{n, m}^{+}$and $\bar{C}_{n}$ is that one wants to keep track not only of the fact that certain points in $\mathcal{H}$, resp. in $\mathbb{R}$, collapse together, or that certain points of $\mathcal{H}$ and $\mathbb{R}$ collapse together to $\mathbb{R}$, but one wants also to record, intuitively, the corresponding rate of convergence. Such compactifications were first thoroughly discussed by Fulton-MacPherson [15] in the algebro-geometric context: Kontsevich [22] adapted the methods of [15] for the configuration spaces of the type $C_{n, m}^{+}$and $C_{n}$.

We introduce first the compactification $\bar{C}_{n}$ of $C_{n}$, which will play an important rôle also in the discussion of the boundary stratification of $\bar{C}_{n, m}^{+}$. We consider the map from Conf ${ }_{n}$ to the product of $n(n-1)$ copies of the circle $S^{1}$, and the product of $n(n-1)(n-2)$ copies of the 2-dimensional real projective space $\mathbb{R P}^{2}$, which is defined explicitly via

$$
\left(z_{1}, \ldots, z_{n}\right) \stackrel{\iota_{n}}{\longmapsto} \prod_{i \neq j} \frac{\arg \left(z_{j}-z_{i}\right)}{2 \pi} \times \prod_{\substack{i \neq j, j \neq k \\ i \neq k}}\left[\left|z_{i}-z_{j}\right|:\left|z_{i}-z_{k}\right|:\left|z_{j}-z_{k}\right|\right] .
$$

$\iota_{n}$ descends in an obvious way to $C_{n}$, and defines an embedding of the latter into a compact manifold. Hence the following definition makes sense.

Definition 6.4. The compactified configuration space $\bar{C}_{n}$ of $n$ points in the complex plane is defined as the closure of the image of $C_{n}$ w.r.t. $\iota_{n}$ in $\left(S^{1}\right)^{n(n-1)} \times\left(\mathbb{R P}^{2}\right)^{n(n-1)(n-2)}$.

Next, we consider the open configuration space $C_{n, m}^{+}$. First of all, there is a natural imbedding of Conf $_{n, m}^{+}$into $\operatorname{Conf}_{2 n+m}$, which is obviously equivariant w.r.t. the action of $G_{2}$,

$$
\left(z_{1}, \ldots, z_{n}, q_{1}, \ldots, q_{m}\right) \stackrel{\iota_{n, m}^{+}}{\longmapsto}\left(z_{1}, \ldots, z_{n}, \overline{z_{1}}, \ldots, \overline{z_{n}}, q_{1}, \ldots, q_{m}\right) .
$$

Moreover, $\iota_{n, m}^{+}$descends to an embedding $C_{n, m}^{+} \rightarrow C_{2 n+m} \cdot{ }^{6}$ We may thus compose $\iota_{n, m}^{+}$ with $\iota_{2 n+m}$ in order to get a well-defined imbedding of $C_{n, m}^{+}$into $\left(S^{1}\right)^{(2 n+m)(2 n+m-1)} \times$ $\left(\mathbb{R P}^{2}\right)^{(2 n+m)(2 n+m-1)(2 n+m-2)}$, which justifies the following definition.

Definition 6.5. The compactified configuration space $\bar{C}_{n, m}^{+}$of $n$ points in $\mathcal{H}$ and $m$ ordered points in $\mathbb{R}$ is defined as the closure of the image w.r.t. to the imbedding $\iota_{2 n+m} \circ \iota_{n, m}^{+}$of $C_{n, m}^{+}$into $\left(S^{1}\right)^{(2 n+m)(2 n+m-1)} \times\left(\mathbb{R P}^{2}\right)^{(2 n+m)(2 n+m-1)(2 n+m-2)}$.

\footnotetext{
${ }^{6}$ To see this, first remember that $G_{3}=G_{2} \ltimes \mathbb{R}$, and then observe that any orbit of $\mathbb{R}$ (acting by simultaneous imaginary translations) intersects $\iota_{n, m}^{+}\left(\operatorname{Conf}_{n, m}^{+}\right)$in at most one point.
} 
We notice that there is an obvious action of $\mathfrak{S}_{n}$, the permutation group of $n$ elements, on $C_{n}$, resp. $C_{n, m}^{+}$, by permuting the points in the complex plane, resp. the $n$ points in $\mathcal{H}$ : the action of $\mathfrak{S}_{n}$ extends to an action on $\bar{C}_{n}$ and $\bar{C}_{n, m}^{+}$. Thus, we may consider more general configuration spaces $C_{A}$ and $C_{A, B}^{+}$, where now $A$ (resp. $B$ ) denotes a finite (resp. ordered) subset of $\mathbb{N}$; they also admit compactifications $\bar{C}_{A}$ and $\bar{C}_{A, B}^{+}$, which are defined similarly as in Definition 6.4 and 6.5.

Another important property of the compactified configuration spaces $\bar{C}_{A}$ and $\bar{C}_{A, B}^{+}$has to do with projections. Namely, for any non-empty subset $A_{1} \subset A$ (resp. pair $A_{1} \subset A, B_{1} \subset B$ such that $\left.A_{1} \sqcup B_{1} \neq \emptyset\right)$ there is a natural projection $\pi_{\left(A, A_{1}\right)}\left(\operatorname{resp} . \pi_{\left(A, A_{1}\right),\left(B, B_{1}\right)}\right)$ from $C_{A}$ onto $C_{A_{1}}$ (resp. from $C_{A, B}$ onto $C_{A_{1}, B_{1}}$ ) given by forgetting the points labelled by indices which are not in $A_{1}$ (resp. not in $\left.A_{1} \sqcup B_{1}\right)$. The projection $\pi_{\left(A, A_{1}\right)}\left(\operatorname{resp} . \pi_{\left(A, A_{1}\right),\left(B, B_{1}\right)}\right)$ extends to a well-defined projection between $\bar{C}_{A}$ and $\bar{C}_{A_{1}}$ (resp. $\bar{C}_{A, B}$ and $\bar{C}_{A_{1}, B_{1}}$ ). Moreover, both projections preserve the boundary stratifications of all compactified configuration spaces involved.

Finally, we observe that the compactified configuration spaces $\bar{C}_{n}$ and $\bar{C}_{n, m}^{+}$inherit both orientation forms from $C_{n}$ and $C_{n, m}^{+}$respectively; the boundary stratifications of both spaces, together with their inherited orientation forms, induce in a natural way orientation forms on all boundary strata. We neglect here the orientation choices of the boundary strata of $\bar{C}_{n}$ and $\bar{C}_{n, m}^{+}$, referring to [3] for all important details.

Examples 6.6. (i) The configuration space $C_{0, m}^{+}$can be identified with the open $(m-2)$ simplex, consisting of $m-2$-tuples $\left(q_{1}, \ldots, q_{m-2}\right)$ in $\mathbb{R}^{m-2}$, such that

$$
0<q_{1}<\cdots<q_{m-2}<1 .
$$

This is possible by means of the free action of the group $G_{2}$ on $\operatorname{Conf}_{0, m}^{+}, m \geq 2$, namely by fixing the first coordinate to 0 by translations and rescale the last one to 1 . However, the compactified space $\bar{C}_{0, m}^{+}$, for $m>3$, does not correspond to the closed simplex $\triangle_{m-2}$ : the strata of codimension 1 of $\triangle_{m-2}$ correspond to the collapse of only two consecutive coordinates, while the strata of codimension 1 of $\bar{C}_{0, m}^{+}$comprise the collapse of a larger number of points. $\bar{C}_{0, m}^{+}$actually is the $(m-2)$-th Stasheff polytope [31].

(ii) The configuration space $C_{1,1}^{+}$can be identified with an open interval: more precisely, by means of the action of $G_{2}$ on Conf $_{1,1}^{+}$, we can fix the point $q_{1}$ in $\mathbb{R}$ to 0 and the modulus of the point $z_{1}$ in $\mathcal{H}$ to be 1 . Hence, $\left.C_{1,1}^{+} \cong S^{1} \cap \mathcal{H} \cong\right] 0,1[$. The corresponding compactified configuration space $\bar{C}_{1,1}^{+}$is simply the closed interval [0,1]: in terms of collapsing points, the two boundary strata correspond to the situation where the point $z_{1}$ in $\mathcal{H}$ tends to the point $q_{1}$ in $\mathbb{R}$ (from the left and right).

(iii) The configuration space $C_{2}$ can be identified with $S^{1}$ : by means of the action of the group $G_{3}$ on Conf $_{2}$, e.g. the first point can be fixed to 0 and its distance to the second point fixed to 1 . Thus, $\bar{C}_{2}=C_{2} \cong S^{1}$.

(iv) The configuration space $C_{2,0}^{+}$can be identified with $\mathcal{H} \backslash\{\mathrm{i}\}$ : by means of the action of $G_{2}$, we can fix e.g. the first point $p_{1}$ in $\mathcal{H}$ to i. The corresponding compactified configuration space $\bar{C}_{2,0}^{+}$is often referred to as Kontsevich's eye: in fact, its graphical depiction resembles to an eye. More precisely, the boundary stratification of $\bar{C}_{2,0}^{+}$consists of three boundary faces of codimension 1 and two boundary faces of codimension 2. In terms of configuration spaces, the boundary faces of codimension 1 are identified with $\bar{C}_{2} \cong S^{1}$ and $\bar{C}_{1,1}^{+} \cong[0,1]$, while the boundary faces of codimension 2 are both identified with $\bar{C}_{0,2}^{+} \cong\{0\}$ : the face $\bar{C}_{2}$, resp. $\bar{C}_{1,1}^{+}$, corresponds to the collapse of both point $z_{1}$ and $z_{2}$ in $\mathcal{H}$ to a single point in $\mathcal{H}$, resp. to the situation where one of the points $z_{1}$ and $z_{2}$ tends to a point in $\mathbb{R}$, while both faces of codimension 2 correspond to the situation where both $p_{1}$ and $p_{2}$ tend to distinct points 
in $\mathbb{R}$. Pictorially, the boundary stratum $\bar{C}_{2}$ corresponds to the pupil of Kontsevich's eye; the boundary strata $\bar{C}_{1,1}^{+}$correspond to Kontsevich's eyelids, and, finally, the codimension 2 strata to the two intersection points of the two eyelids.

For the sake of simplicity, from now on, points in $\mathcal{H}$, resp. $\mathbb{R}$, are said to be of the first, resp. second type.

Description of a few boundary components.

Now, for the main computations of Section 8, we need mostly only boundary strata of codimension 1 and, in Subsection 7.3, particular boundary strata of codimension 2 of $\bar{C}_{n, m}^{+}$: we list here the relevant boundary strata of codimension 1 and of codimension 2, which are needed. For the boundary strata of codimension 1, we are concerned with two situations:

i) For a subset $A \subset\{1, \ldots, n\}$, the points $z_{i}$ of the first type, $i \in A$, collapse together to a single point of the first type; more precisely, we have the factorization of the boundary stratum

$$
\partial_{A} \bar{C}_{n, m}^{+} \cong \bar{C}_{A} \times \bar{C}_{n-|A|+1, m}^{+}
$$

here, $2 \leq|A|$ denotes the cardinality of the subset $A$. Intuitively, $\bar{C}_{A}$ describes the configurations of distinct points of the first type in $\bar{C}_{n, m}^{+}$which collapse to a single point of the first type.

ii) For a subset $A \subset\{1, \ldots, n\}$ and an ordered subset $B \subset\{1, \ldots, m\}$ of consecutive integers, the points of the first type $z_{i}, i \in A$, and the points of the second type $q_{i}$ in $\mathbb{R}$ collapse to a single point of the second type; more precisely, we have the factorization

$$
\partial_{A, B} \bar{C}_{n, m}^{+} \cong \bar{C}_{A, B}^{+} \times \bar{C}_{n-|A|, m-|B|+1}^{+} .
$$

Intuitively, $\bar{C}_{A, B}^{+}$describes the configurations of points of the first type and of the second type in $\bar{C}_{n, m}^{+}$, which collapse together to a single point of the second type.

As for the codimension 2 boundary strata, which will be of importance to us, we have the following situation: there exist disjoint subsets $A_{1}, A_{2}$ of $\{1, \ldots, n\}$, and disjoint ordered subsets $B_{1}, B_{2}$ of $\{1, \ldots, m\}$ of consecutive integers, such that the corresponding boundary stratum of codimension 2 admits the factorization

$$
\bar{C}_{A_{1}, B_{1}}^{+} \times \bar{C}_{A_{2}, B_{2}}^{+} \times \bar{C}_{n-\left|A_{1}\right|-\left|A_{2}\right|, m-\left|B_{1}\right|-\left|B_{2}\right|+2}^{+} .
$$

Intuitively, $\bar{C}_{A_{1}, B_{1}}^{+}$and $\bar{C}_{A_{2}, B_{2}}^{+}$parametrize disjoint configurations of points of the first and of the second type, which collapse together to two distinct points of the first type. We will write later on such a boundary stratum a bit differently, namely, after reordering of the points after collapse, the third factor in the previous factorization can be written as $\bar{C}_{A_{3}, B_{3}}^{+}$, for a subset $A_{3}$ of $\{1, \ldots, n\}$ of cardinality $n-\left|A_{1}\right|-\left|A_{2}\right|$, for an ordered subset $B_{3}$ of $\{1, \ldots, m\}$ of cardinality $m-\left|B_{1}\right|-\left|B_{2}\right|+2$.

\subsection{Directed graphs and integrals over configuration spaces.}

The standard angle function.

We introduce now the standard angle function ${ }^{7}$. For this purpose we consider a pair of distinguished points $(z, w)$ in $\mathcal{H} \sqcup \mathbb{R}$ and we denote by $\varphi(z, w)$ the normalized hyperbolic angle in $\mathcal{H} \sqcup \mathbb{R}$ between $z$ and $w$; more explicitly,

$$
\varphi(z, w)=\frac{1}{2 \pi} \arg \left(\frac{w-z}{w-\bar{z}}\right) .
$$

\footnotetext{
${ }^{7}$ As observed by Kontsevich [22] one could in principle choose more general angle functions, starting from the abstract properties of the standard angle function.
} 
Observe that the assignement $C_{2,0}^{+} \ni(z, w) \longmapsto \varphi(z, w) \in S^{1}$ obviously extends to a smooth map from $\bar{C}_{2,0}^{+}$to $S^{1}$, which enjoys the following properties (these properties play an important rôle in the computations of Sections 7 and 8):

i) the restriction of $\varphi$ to the boundary stratum $\bar{C}_{2} \cong S^{1}$ equals the standard angle coordinate on $S^{1}$ added by $\frac{\pi}{2}$;

ii) the restriction of $\varphi$ to the boundary stratum $\bar{C}_{1,1}^{+}$, corresponding to the upper eyelid of Kontsevich's eye, vanishes.

We will refer to $\varphi$ as to the angle function.

Integral weights associated to graphs.

We consider, for given positive integers $n$ and $m$, directed graphs $\Gamma$ with $m+n$ vertices labelled by the set $\mathcal{E}(\Gamma)=\{1, \ldots, n, \overline{1}, \ldots, \bar{m}\}$. Here, "directed" means that each edge of $\Gamma$ carries an orientation. Additionally, the graphs we consider are required to have no loop (a loop is an edge beginning and ending at the same vertex).

To any edges $e=(i, j) \in \mathcal{E}(\Gamma)$ of such a directed graph $\Gamma$, we associate the smooth map

$$
\varphi_{e}: C_{n, m}^{+} \longrightarrow S^{1} ;\left(z_{1}, \ldots, z_{n}, z_{\overline{1}}, \ldots, z_{\bar{m}}\right) \longmapsto \varphi\left(z_{i}, z_{j}\right)
$$

which obviously extends to a smooth map from $\bar{C}_{n, m}^{+}$to $S^{1}$.

To any directed graph $\Gamma$ without loop and with $\mathcal{E}(\Gamma)=\{1, \ldots, n, \overline{1}, \ldots, \bar{m}\}$ as set of vertices, we then associate a differential form

$$
\omega_{\Gamma}:=\bigwedge_{e \in \mathcal{E}(\Gamma)} \mathrm{d} \varphi_{e}
$$

on the (compactified) configuration space $\bar{C}_{n, m}^{+}$.

Remark 6.7. We observe that, a priori, it is necessary to choose an ordering of the edges of $\Gamma$ since $\omega_{\Gamma}$ is a product of 1-forms: two different orderings of the edges of $\Gamma$ simply differ by a sign. This sign ambiguity precisely coincide (and thus cancel) with the one appearing in the definition of $B_{\Gamma}$, as it is pointed out in Remark 5.7.

We recall from Subsection 6.1 and 6.2 that $\bar{C}_{n, m}^{+}$is orientable, and that the orientation of $C_{n, m}^{+}$specifies an orientation for any boundary stratum thereof.

Definition 6.8. The weight $\mathcal{W}_{\Gamma}$ of the directed graph $\Gamma$ is

$$
\mathcal{W}_{\Gamma}:=\int_{\bar{C}_{n, m}^{+}} \omega_{\Gamma} .
$$

Observe that the weight (6.2) truly exists since it is an integral of a smooth differential form over a compact manifold.

\section{Vanishing lemmatas.}

It follows immediately from the definition of $\mathcal{W}_{\Gamma}$ that it is non-zero only if

- the cardinality of $\mathcal{E}(\Gamma)$ equals $2 n+m-2$ (i.e. $\omega_{\Gamma}$ is a top degree form),

- $\Gamma$ has no double edge (i.e. two edges with the same source and same target),

- second type vertices do not have outgoing edges.

In particular, $\mathcal{W}_{\Gamma}$ is non-zero only if $\Gamma \in \mathcal{G}_{n, m}$.

For later purposes, we need a few non-trivial vanishing Lemmata concerning the weights, which we use later on in Sections 7, 8 and 9.

Lemma 6.9. If $\Gamma$ in $\mathcal{G}_{n, m}$ has a bivalent vertex $v$ of the first type with exactly one incoming and exactly one outgoing edge (see Figure 2), then its weight $\mathcal{W}_{\Gamma}$ vanishes. 


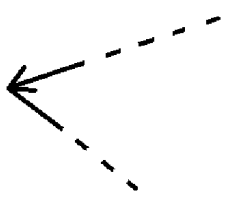

Figure 2

We observe that the target of the outgoing edge may be of the first or of the second type, while the source of the incoming edge must be of the first type.

Sketch of proof. We consider exemplarily the case, where both vertices $v_{1}$ and $v_{2}$ are of the first type; the corresponding points in $\bar{C}_{n, m}^{+}$are denoted by $z_{1}$ and $z_{2}$ respectively.

Using Fubini's Theorem, we isolate in the weight $\mathcal{W}_{\Gamma}$ the factor

$$
\int_{\mathcal{H} \backslash\left\{z_{1}, z_{2}\right\}} \mathrm{d} \varphi_{e_{1}} \wedge \mathrm{d} \varphi_{e_{2}} .
$$

The rest of the proof consists in showing that (6.3) vanishes.

We observe that (6.3) is a function depending on $\left(z_{1}, z_{2}\right)$. We first show that it is a constant function. Namely, (6.3) is the integral along the fiber of the integrand form w.r.t. the natural projection $\bar{C}_{3,0}^{+} \stackrel{\pi}{\rightarrow} \bar{C}_{2,0}^{+} ;\left(z_{1}, z_{2}, z_{3}\right) \mapsto\left(z_{1}, z_{2}\right)$ : independence of $z_{1}$ and $z_{2}$ follows by means of the generalized Stokes' Theorem

$$
\mathrm{d}\left(\pi_{*}\left(\mathrm{~d} \varphi_{e_{1}} \wedge \mathrm{d} \varphi_{e_{2}}\right)\right)= \pm \pi_{*}\left(\mathrm{~d}\left(\mathrm{~d} \varphi_{\left(v_{1}, v\right)} \wedge \mathrm{d} \varphi_{\left(v, v_{2}\right)}\right)\right) \pm \pi_{\partial *}\left(\mathrm{~d} \varphi_{e_{1}} \wedge \mathrm{d} \varphi_{e_{2}}\right),
$$

where the second term on the right hand-side corresponds to the boundary contributions coming from fiber integration. Since the integrand is obviously closed, it remains to show the vanishing of the boundary contributions. It is clear that there are four boundary strata of codimension 1 of the fibers of $\pi$, namely, when $i$ ) the point $z$ (corresponding to the vertex $v$ ) approaches $z_{1}$ or $z_{2}, i i$ ) when $z$ approaches $\mathbb{R}$, and $i i i$ ) when $z$ tends to infinity (which must be viewed as a half-circle, whose radius tends to infinity). The properties of the angle function imply that the contributions coming from ii) and iii) vanish, and that the two contributions coming from $i$ ) cancel together.

Hence, we may choose e.g. $z_{1}=\mathrm{i}$ and $z_{2}=2 \mathrm{i}$ : for this particular choice, the involution $z \mapsto-\bar{z}$ of $\mathcal{H} \backslash\{\mathrm{i}, 2 \mathrm{i}\}$ reverses the orientation of the fibers, but preserves the integrand form, whence the claim follows.

Lemma 6.10. For a positive integer $n \geq 3$, the integral over $\bar{C}_{n}$ of the product of $2 n-3$ forms of the type $\mathrm{d}\left(\arg \left(z_{i}-z_{j}\right)\right), i \neq j$, vanishes.

Proof. The proof relies on an analytic argument, which involves a tricky computation with complex logarithms; for a complete proof we refer to [22] and [20] (see also [9, appendix]). 


\section{The MAP $\mathcal{U}_{Q}$ AND ITS PROPERTIES}

In this section we stress out and prove remarkable properties of the map $\mathcal{U}_{Q}$ defined by the formula (5.2). Namely, we first prove that $\mathcal{U}_{Q}$ is a quasi-isomorphism of complexes, and we then give, for any polyvecor fields $\alpha, \beta$, explicit formulae for $\mathcal{U}_{Q}(\alpha \wedge \beta)$ and $\mathcal{U}_{Q}(\alpha) \cup \mathcal{U}_{Q}(\beta)$ in terms of new weights associated to graphs.

The proof follows closely the treatment of Manchon and Torossian [26], and strongly uses the remarkably rich cominatorics of the boundary of the compactified configuration spaces introduced in the previous section.

\subsection{The quasi-isomorphism property.}

This Subsection is devoted to the proof of the following result.

Proposition 7.1. The map $\mathcal{U}_{Q}: T_{\text {poly }} V \longrightarrow D_{\text {poly }} V$ defined by equation (5.2) is a quasiisomorphism of complexes, in the sens that for any polyvector field $\alpha$

$$
\mathcal{U}_{Q}(Q \cdot \alpha)=\left(d_{H}+Q \cdot\right)\left(\mathcal{U}_{Q}(\alpha)\right)
$$

and $\mathcal{U}_{Q}$ induces an isomorphism of graded vector spaces on cohomology.

Sketch of the proof. We first sketch the proof of equation (7.1). The fact that it induces an isomorphism in cohomology then follows from a straightforward spectral sequence argument.

Let $\Gamma \in \mathcal{G}_{n+1, m+1}$ be a graph with $2 n+m$ edges, the first type vertex 1 having exactly $m$ outgoing edges, and all other first type vertices having a single outgoing edge. We then apply the Stokes' Theorem

$$
\int_{\partial \bar{C}_{n+1, m+1}^{+}} \omega_{\Gamma}=\int_{C_{n+1, m+1}^{+}} \mathrm{d} \omega_{\Gamma}=0 .
$$

and discuss the meaning of the following resulting identity: for any poly-vector field $\alpha$ with $m$ arguments, and any functions $f_{1}, \ldots, f_{m+1}$,

$$
\sum_{C} \pm \sum_{\Gamma \in \mathcal{G}_{n+1, m+1}}\left(\int_{C} \omega_{\Gamma}\right) B_{\Gamma}(\alpha, \underbrace{Q, \ldots, Q}_{n \text { times }})\left(f_{1}, \ldots, f_{m+1}\right)=0 .
$$

Here $C$ runs over all codimension 1 boundary components of $\bar{C}_{n+1, m+1}^{+}$, and the sign depends on the induced orientation from $\bar{C}_{n+1, m+1}^{+}$. We now discuss the possible non trivial contributions of each bouldary component $C$. Using Fubini's Theorem we find (up to signs coming from orientation choices) the following factorization property:

$$
\int_{C} \omega_{\Gamma}=\int_{C_{\mathrm{int}}} \omega_{\Gamma_{\mathrm{int}}} \int_{C_{\mathrm{out}}} \omega_{\Gamma_{\mathrm{out}}},
$$

where $\Gamma_{\text {int }}$ (resp. $\Gamma_{\text {out }}$ ) is the subgraph of $\Gamma$ whose edges are those with both source and target lying in the subset of collapsing points (resp. is the quotient graph of $\Gamma$ by its subgraph $\Gamma_{\text {int }}$ ).

Let us begin with the boundary components of the form $C=\partial_{A} \bar{C}_{n+1, m+1}^{+}($with $|A| \geq 2)$. It follows from the vanishing Lemma 6.10 that there is no contribution if $|A| \geq 3$. If $|A|=2$ then $\Gamma_{\text {int }}$ consists of a single edge and the first factor in the factorization on the r.h.s. of (7.2) equals 1 . There are two possbilities:

- either $1 \notin A$ and thus, taking the sum of the contributions of all graphs $\Gamma$ leading to the same pair $\left(\Gamma_{\text {int }}, \Gamma_{\text {out }}\right)$, one obtains something proportional to

$$
\mathcal{W}_{\Gamma_{\text {out }}} B_{\Gamma_{\text {out }}}(\alpha, Q, \ldots, \underbrace{Q \circ Q}_{=0}, \ldots, Q)\left(f_{1}, \ldots, f_{m+1}\right)=0 .
$$


- or $1 \in A$ and thus, again taking the sum of the contributions of all graphs $\Gamma$ leading to the same pair $\left(\Gamma_{\text {int }}, \Gamma_{\text {out }}\right)$, and adding up the terms coming from the same graphs $\Gamma$ after reversing the unique arrow of $\Gamma_{\text {int }}$, one obtains

$$
\mathcal{W}_{\Gamma_{\text {out }}} B_{\Gamma_{\text {out }}}(Q \cdot \alpha, \underbrace{Q, \ldots, Q}_{n-1 \text { times }})\left(f_{1}, \ldots, f_{m+1}\right) .
$$

We then continue with the boundary components of the form $C=\partial_{A, B} \bar{C}_{n+1, m+1}^{+}$. Again there are two possibilities:

- either $1 \notin A$ and thus $2|A|+|B|-2=|A|$, i.e. $|A|+|B|=2$. Hencefore the graph $\Gamma_{\text {int }}$ can belong to one of the following three types:

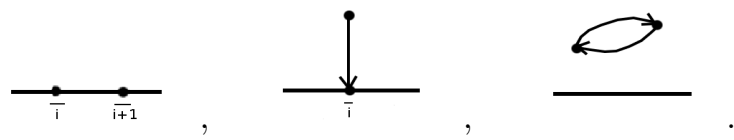

Summing the contributions of all graphs leading to the same pair $\left(\Gamma_{\text {int }}, \Gamma_{\text {out }}\right)$, one obtains

$$
\mathcal{W}_{\Gamma_{\text {out }}} B_{\Gamma_{\text {out }}}(\alpha, \underbrace{Q, \ldots, Q}_{n \text { times }})\left(f_{1}, \ldots, f_{i} f_{i+1}, \ldots, f_{m+1}\right)
$$

for the first type of graphs, and

$$
\mathcal{W}_{\Gamma_{\text {out }}} B_{\Gamma_{\text {out }}}(\alpha, \underbrace{Q, \ldots, Q}_{n-1 \text { times }})\left(f_{1}, \ldots, Q \cdot f_{i}, \ldots, f_{m+1}\right)
$$

for the second one. The third type of graph does not contribute since its weight is zero thanks to the vanishing Lemma 6.9.

- or $1 \in A$ and thus $2(n+1-|A|)+m-|B|=n+1-|A|$, i.e. $|A|+|B|=n+1+m$. Hencefore $\Gamma_{\text {out }}$ must be one the following two graphs:

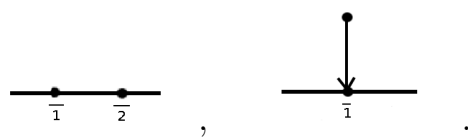

The corresponding contributions (after summing over graphs leading to the same decomposition) respectively are

$\mathcal{W}_{\Gamma_{\text {int }}}\left(f_{1} B_{\Gamma_{\text {int }}}(\alpha, \underbrace{Q, \ldots, Q}_{n \text { times }})\left(f_{2}, \ldots, f_{m+1}\right) \pm B_{\Gamma_{\text {int }}}(\alpha, \underbrace{Q, \ldots, Q}_{n \text { times }})\left(f_{1}, \ldots, f_{m}\right) f_{m+1}\right)$

for the first one and

$$
\mathcal{W}_{\Gamma_{\mathrm{int}}} Q \cdot\left(B_{\Gamma_{\mathrm{int}}}(\alpha, \underbrace{Q, \ldots, Q}_{n-1 \text { times }})\left(f_{1}, \ldots, f_{m+1}\right)\right)
$$

for the second one.

We now summarize all non trivial contributions: (7.4) gives the l.h.s. of equation (7.1), (7.6) together with (7.4) gives $d_{H} \mathcal{U}_{Q}(\alpha)$, and (7.7) together with (7.5) gives $Q \cdot \mathcal{U}_{Q}(\alpha)$. Therefore equation (7.1) is satisfied and it remains to prove that $\mathcal{U}_{Q}$ induces an isomorphism on the level of cohomology. To do so we consider the mapping cône $C_{Q}^{\bullet}$ of $\mathcal{U}_{Q}$ together with the decreasing filtration on it coming from the grading on $T_{\text {poly }} V$ and $D_{\text {poly }} V$ induced by the degree we have denoted by $|\cdot|$ in Section 5 . The zero-th term of the corresponding spectral sequence is given by the mapping cône of the Hochschild-Kostant-Rosenberg map $I_{H K R}:\left(T_{\text {poly }} V, 0\right) \longrightarrow\left(D_{\text {poly }} V, d_{H}\right)$, and thus $E_{1}=\{0\}$ (as $I_{H K R}$ is a quasi-isomorphism). This ends the proof of the Proposition. 


\subsection{The cup product on polyvector fields.}

In this Subsection, we consider the cup product between any two polyvector fields $\alpha$ and $\beta$ : we want to express the result of applying (5.2) on the cup product $\alpha \wedge \beta$ in terms of integral weights over a suitable submanifold $\mathcal{Z}_{0} \subset \bar{C}_{n+2, m}^{+}$, that we define now.

We recall from Subsection 6.2 that the compactified configuration space $\bar{C}_{2,0}^{+}$can be pictured as Kontsevich's eye. We choose a point $x \in C_{2} \subset \bar{C}_{n+2, m}^{+}$. Furthermore, for any two positive integers $n$ and $m$ we consider the projection $F:=\pi_{\{1,2\}, \emptyset}$ from $\bar{C}_{n+2, m}^{+}$onto $\bar{C}_{2,0}^{+}$, using the same notations as in Subsection 6.2 . Then we denote by $\mathcal{Z}_{0}$ the submanifold of $\bar{C}_{n+2, m}^{+}$given by the preimage w.r.t. $F$ of the point $x$; accordingly, to a graph $\Gamma \in \mathcal{G}_{n+2, m}$ we associate a new weight $\mathcal{W}_{\Gamma}^{0}$ given by

$$
\mathcal{W}_{\Gamma}^{0}:=\int_{\mathcal{Z}_{0}} \omega_{\Gamma}
$$

using the same notations as in paragraph 6.3.

Proposition 7.2. For any two polyvector fields $\alpha$ and $\beta$ on $V$, the following identity holds true:

$$
\mathcal{U}_{Q}(\alpha \wedge \beta)=\sum_{n \geq 0} \frac{1}{n !} \sum_{\Gamma \in \mathcal{G}_{n+2, m}} \mathcal{W}_{\Gamma}^{0} B_{\Gamma}(\alpha, \beta, \underbrace{Q, \ldots, Q}_{n \text { times }}) .
$$

Notice in particular that the r.h.s. of (7.8) does not depend on the choice of $x \in C_{2}$.

Proof. We split the proof into many substeps.

Lemma 7.3. If, in a graph $\Gamma \in \mathcal{G}_{n+2, m}$, the two vertices of the first type labelled by 1 and 2 (corresponding to where the polyvector fields $\alpha$ and $\beta$ have been put) are linked by at least one edge, then $\mathcal{W}_{\Gamma}^{0}$ vanishes.

Proof. The main argument of the proof is that $\mathcal{Z}_{0}$ intersects non-trivially only those boundary strata of codimension 1 , where a certain number of points of the first type in $\bar{C}_{n+2, m}^{+}$ collapse to a point of the first type. Recalling the arguments at the end of Subsection 6.2, such boundary strata are of the form $\partial_{A} \bar{C}_{n+2, m}^{+} \cong \bar{C}_{A} \times \bar{C}_{n-|A|+3, m}^{+}, A$ being a subset of $\{1, \ldots, n+2\}$ of cardinality $|A| \geq 2$. Moreover, since $x$ lies in $\bar{C}_{2}$, such boundary strata correspond to subsets $A$, which contain both vertices labelled by 1 and 2 .

Using Fubini's Theorem, we find (up to signs coming from orientation choices) the following factorization of $\mathcal{W}_{\Gamma}^{0}$ :

$$
\int \omega_{\mathcal{Z}_{0} \cap \partial_{A} \bar{C}_{n+2, m}^{+}}=\int \omega_{\mathcal{Z}_{0} \cap \bar{C}_{A}} \int_{\bar{C}_{n-|A|+3, m}^{+}} \omega_{\Gamma_{\text {out }}} .
$$

Here we keep the same notation as in the previous Subsection for $\Gamma_{\text {int }}$ and $\Gamma_{\text {out }}$. We also observe that, since $x \in C_{2}$, then $\mathcal{Z}_{0} \cap\left(\bar{C}_{A} \times \bar{C}_{n-|A|+3, m}^{+}\right)$is a product $C^{\prime} \times \bar{C}_{n-|A|+3, m}^{+}$, with $C^{\prime} \subset \bar{C}_{A}$. By abuse of notation, and for the sake of simplicity, we have wrote $\mathcal{Z}_{0} \cap \bar{C}_{A}$ for the submanifold $C^{\prime}$ in $\bar{C}_{A}$. We use this notation many times below.

If there is at least one edge connecting 1 and 2 , which correspond in $\mathcal{Z}_{0}$ to the fixed point $x$ in $C_{2} \subset \bar{C}_{2,0}^{+}$, then, in the form $\omega_{\Gamma_{\text {int }}}$, there is at least one 1-form of the type $\mathrm{d}\left(\arg \left(z_{2}-z_{1}\right)\right)$ or $\mathrm{d}\left(\arg \left(z_{2}-\bar{z}_{1}\right)\right)$ (using the notations from Subsection 6.1), which vanish, since the arguments remains constant in $\mathcal{Z}_{0} \cap \bar{C}_{A}$. Hence, the claim follows.

Lemma 7.4. If the graph $\Gamma \in \mathcal{G}_{n+2, m}$ contains no edge connecting the vertices of the first type 1 and 2 , then

$$
\mathcal{W}_{\Gamma}^{0}=\mathcal{W}_{\widetilde{\Gamma}}
$$

where $\widetilde{\Gamma}$ is the graph in $\mathcal{G}_{n+1, m}$ obtained from $\Gamma$ by collapsing the vertices 1 and 2 . 
Proof. We recall from Lemma 7.3 that $\mathcal{Z}_{0}$ intersects non-trivially only boundary strata of the form $\bar{C}_{A} \times \bar{C}_{n-|A|+3, m}^{+}$, where $A$ is a subset of $\{1, \ldots, n+2\}$ and containing 1 and 2 . Using again Fubini's Theorem, we obtain

$$
\mathcal{W}_{\Gamma}^{0}=\int_{\mathcal{Z}_{0} \cap \partial_{A} \bar{C}_{n+2, m}^{+}} \omega_{\Gamma}=\int_{\mathcal{Z}_{0} \cap \bar{C}_{A}} \omega_{\Gamma_{\text {int }}} \int \omega_{\bar{C}_{n-|A|+3, m}^{+}} \omega_{\Gamma_{\text {out }}} .
$$

The points corresponding to the vertices 1 and 2 of the first type are fixed by assumption.

By dimensional reasons, the only (possibly) non-trivial contributions to the first factor in the factorization on the right hand-side of (7.9) occur only if the degree of the integrand $\omega_{\Gamma_{\text {int }}}$ equals $2|A|-4$. The corresponding integral vanishes by the the arguments in the proof of Kontsevich's Lemma 6.10, for which we refer to [22]: suffice it to mention that, in the proof in [22], Kontsevich reduces the case of the integral over $\bar{C}_{n}$ of a product of $2 n-3$ forms to the case of the integral over a manifold of the form $\mathcal{Z}_{0} \cap \bar{C}_{n}$ (i.e. he fixes two vertices) and then he extracts from the integrand the 1-form, corresponding to the edge joining the two fixed points (i.e. there is no edge between the two fixed vertices). Then, he shows that the latter integral vanishes by complicated analytical arguments (tricks with logarithms and distributions): anyway, the very same arguments imply that the first factor in the factorization (7.9) vanishes.

Hence, we are left with the case $|A|=2$, i.e. $A=\{1,2\}$ : therefore, we obtain, again using Fubini's Theorem,

$$
\mathcal{W}_{\Gamma}^{0}=\int_{\mathcal{Z}_{0} \cap \partial_{\{1,2\}} \bar{C}_{n+2, m}^{+}} \omega_{\Gamma}=\int_{\mathcal{Z}_{0} \cap \bar{C}_{2}} \omega_{\Gamma_{\text {int }}} \int_{\bar{C}_{n+1, m}^{+}} \omega_{\Gamma_{\text {out }}} .
$$

It is clear that $\Gamma_{\text {out }}$ is exactly the graph $\widetilde{\Gamma} \in \mathcal{G}_{n+1, m}$ in the claim of the Lemma. On the other hand, by properties of the angle function, when restricted to the boundary stratum $\bar{C}_{2}$, we have

$$
\int_{\mathcal{Z}_{0} \cap \bar{C}_{2}} \omega_{\Gamma_{\text {int }}}=1
$$

observing that $\Gamma_{\text {int }}$ consists of two vertices of the first type, with no edge connecting them. Thus, we have proved the claim.

We consider now, for a graph $\widetilde{\Gamma}$ in $\mathcal{G}_{n+1, m}$ and with $\alpha, \beta$ and $Q$ as before, the polydifferential operator $B_{\widetilde{\Gamma}}(\alpha \wedge \beta, Q, \ldots, Q)$, where there are $n$ cohomological vector fields $Q$. By the very construction of $B_{\Gamma}$ and by the definition of $\wedge$, we have

$$
B_{\widetilde{\Gamma}}(\alpha \wedge \beta, Q, \ldots, Q)=\sum_{\mathcal{G}_{n+2, m} \ni \Gamma \mapsto \widetilde{\Gamma}} B_{\Gamma}(\alpha, \beta, Q, \ldots, Q),
$$

where the sum is over all possible graphs $\Gamma$ in $\mathcal{G}_{n+2, m}$, which are obtained from $\widetilde{\Gamma}$ by separating the vertices 1 and 2 of the first type without inserting any edge between them; it is clear that contraction of the vertices 1 and 2 of a graph $\Gamma$ as before gives the initial graph $\widetilde{\Gamma}$. This collapsing process is symbolized by the writing $\Gamma \mapsto \widetilde{\Gamma}$.

We finally compute

$$
\begin{aligned}
\sum_{\widetilde{\Gamma} \in \mathcal{G}_{n+1, n}} \mathcal{W}_{\widetilde{\Gamma}} B_{\widetilde{\Gamma}}(\alpha \wedge \beta, Q, \ldots, Q) & =\sum_{\widetilde{\Gamma} \in \mathcal{G}_{n+1, m}} \sum_{\mathcal{G}_{n+2, m} \ni \Gamma \mapsto \widetilde{\Gamma}} \mathcal{W}_{\widetilde{\Gamma}} B_{\Gamma}(\alpha, \beta, Q, \ldots, Q) \\
& =\sum_{\widetilde{\Gamma} \in \mathcal{G}_{n+1, m}} \sum_{\mathcal{G}_{n+2, m} \ni \Gamma \mapsto \widetilde{\Gamma}} \mathcal{W}_{\Gamma}^{0} B_{\Gamma}(\alpha, \beta, Q, \ldots, Q) \\
& =\sum_{\Gamma \in \mathcal{G}_{n+2, m}} \mathcal{W}_{\Gamma}^{0} B_{\Gamma}(\alpha, \beta, Q, \ldots, Q) .
\end{aligned}
$$


The second equality follows from Lemma 7.4, and the third equality is a consequence of Lemma 7.3. This ends the proof of the Proposition.

\subsection{The cup product on polydifferential operators.}

Applying $\mathcal{U}_{Q}$ on polydifferential operators $\alpha$ and $\beta$, we may then take their cup product in the Hochschild complex of polydifferential operators. We want to show that, in analogy with Proposition 7.2, this product can be expressed in terms of integral weights over a suitable submanifold $\mathcal{Z}_{1}$ of $\bar{C}_{n+2, m}^{+}$, that we define now.

Let $y$ be the unique point sitting in the copy of $C_{0,2}^{+}$inside $\partial \bar{C}_{2,0}^{+}$in which the vertex 1 stays on the left of the vertex 2 . Then for any two positive integers $n$ and $m$, using the same notations as in the previous Subsection, we define $\mathcal{Z}_{1}:=F^{-1}(y) \subset \bar{C}_{n+2, m}^{+}$and

$$
\mathcal{W}_{\Gamma}^{1}:=\int_{\mathcal{Z}_{1}} \omega_{\Gamma}
$$

Proposition 7.5. Under the same assumptions of Proposition 7.2, the following identity holds true:

$$
\mathcal{U}_{Q}(\alpha) \cup \mathcal{U}_{Q}(\beta)=\sum_{n \geq 0} \frac{1}{n !} \sum_{\Gamma \in \mathcal{G}_{n+2, m}} \mathcal{W}_{\Gamma}^{1} B_{\Gamma}(\alpha, \beta, \underbrace{Q, \ldots, Q}_{n \text { times }}) .
$$

Proof. First of all, in the definition of $\mathcal{U}_{Q}$, we may consider only those graphs $\Gamma$ in $\mathcal{G}_{n+1, m}$, which do not contain a bivalent vertex as in the assumptions of Lemma 6.9. Since $Q$ is a vector field, putting it on a vertex of the first type means that from the chosen vertex there is only one outgoing edge: the previous observation forces the first type vertices of $\Gamma$ that are not 1 to have more than one incoming edge. Hence, the only first type vertex in a contributing graph $\Gamma$ that can be linked to a second type vertex is 1 . We denote by $\widetilde{\mathcal{G}}_{n+1, m} \subset \mathcal{G}_{n+1, m}$ the subset of graphs having this property.

By the very definition of the cup product in Hochschild cohomology, we obtain

$$
\begin{aligned}
\mathcal{U}_{Q}(\alpha) \cup \mathcal{U}_{Q}(\beta) & =\sum_{k, l \geq 0} \frac{1}{k ! l !} \sum_{\substack{\Gamma_{1} \in \tilde{\mathcal{G}}_{k+1, m_{1}} \\
\Gamma_{2} \in \tilde{\mathcal{G}}_{l+1, m_{2}}}} \mathcal{W}_{\Gamma_{1}} \mathcal{W}_{\Gamma_{2}} B_{\Gamma_{1}}(\alpha, \underbrace{Q, \ldots, Q}_{k \text { times }}) B_{\Gamma_{2}}(\alpha, \underbrace{Q, \ldots, Q}_{l \text { times }}) \\
& =\sum_{k, l \geq 0} \frac{1}{k ! l !} \sum_{\substack{\Gamma_{1} \in \widetilde{\mathcal{G}}_{\mathcal{F}_{1+1}, m_{1}} \\
\Gamma_{2} \in \tilde{\mathcal{G}}_{l+1, m_{2}}}} \mathcal{W}_{\Gamma_{1} \sqcup \Gamma_{2}} B_{\Gamma_{1} \sqcup \Gamma_{2}}(\alpha, \beta, \underbrace{Q, \ldots, Q}_{k+l \text { times }})
\end{aligned}
$$

where, for any graphs $\Gamma_{1} \in \widetilde{\mathcal{G}}_{k+1, m_{1}}$ and $\Gamma_{2} \in \widetilde{\mathcal{G}}_{l+1, m_{2}}$, we have denoted by $\Gamma_{1} \sqcup \Gamma_{2}$ their disjoint union: it is again a graph in $\mathcal{G}_{k+l+2, m_{1}+m_{2}}$. The vertices of $\Gamma_{1} \sqcup \Gamma_{2}$ are re-numbered starting from the numberings of the vertices of $\Gamma_{1}$ and $\Gamma_{2}$ to guarantee the last equality in the previous chain of identities: namely, denoting by an index $i=1,2$ the graph to which belongs a given vertex labelled by $i$, the new numbering of the vertices of $\Gamma_{1} \sqcup \Gamma_{2}$ is

$$
\left\{1_{1}, 1_{2}, 2_{1}, 3_{1}, \ldots,(k+1)_{1}, 2_{2}, 3_{2}, \ldots,(l+1)_{2}\right\} .
$$

Lemma 7.6. If $\Gamma=\Gamma_{1} \sqcup \Gamma_{2} \in \mathcal{G}_{n, m}$, with $\Gamma_{1} \in \widetilde{\mathcal{G}}_{k+1, m_{1}}$ and $\Gamma_{2} \in \widetilde{\mathcal{G}}_{l+1, m_{2}}$, then

$$
\mathcal{W}_{\Gamma}^{1}=\mathcal{W}_{\Gamma_{1}} \mathcal{W}_{\Gamma_{2}}
$$

For any other graph $\Gamma$ in $\mathcal{G}_{n+2, m}, \mathcal{W}_{\Gamma}=0$.

Proof. It follows from its very definition that $\mathcal{Z}_{1}$ intersects non-trivially only those boundary strata $\partial_{T} \bar{C}_{n+2, m}^{+}$of $\bar{C}_{n+2, m}^{+}$of codimension 2 which possess the following factorization, according to Subsection 6.2:

$$
\bar{C}_{A_{1}, B_{1}}^{+} \times \bar{C}_{A_{2}, B_{2}}^{+} \times \bar{C}_{A_{3}, B_{3}}^{+}
$$


where the vertex 1 and the vertex 2 lie in $\bar{C}_{A_{1}, B_{1}}^{+}$and $\bar{C}_{A_{2}, B_{2}}^{+}$respectively; finally, the positive integers $n_{i}:=\left|A_{i}\right|$ and $m_{i}:=\left|B_{i}\right|$ obviously satisfy

$$
n_{1}+n_{2}+n_{3}=n+2 \quad \text { and } \quad m_{1}+m_{2}+\left(m_{3}-2\right)=m .
$$

For a graph $\Gamma \in \mathcal{G}_{n+2, m}$, we denote by $\Gamma_{\text {int }}^{1}$, resp. $\Gamma_{\text {int }}^{2}$, resp. $\Gamma_{\text {out }}$, the subgraph of $\Gamma$, whose vertices are labelled by $A_{1} \sqcup B_{1}$, resp. $A_{2} \sqcup B_{2}$, resp. by contracting the subgraphs $\Gamma_{\text {int }}^{1}$ and $\Gamma_{\text {int }}^{2}$ to two distinct vertices of the second type.

Using Fubini's Theorem once again, we get

$$
\int_{\mathcal{Z}_{1} \cap \partial_{T} \bar{C}_{n+2, m}^{+}} \omega_{\Gamma}=\int_{\bar{C}_{A_{1}, B_{1}}^{+}} \omega_{\Gamma_{\text {int }}^{1}} \int_{\bar{C}_{A_{2}, B_{2}}^{+}} \omega_{\Gamma_{\text {int }}^{2}} \int_{\bar{C}_{A_{3}, B_{3}}^{+}} \omega_{\Gamma_{\text {out }}} .
$$

By the properties of the angle function, there cannot be vertices of $\Gamma_{\text {int }}^{1}$ or $\Gamma_{\text {int }}^{2}$, from which departs an external edge, i.e. an edge whose target lies in set of vertices of $\Gamma_{\text {out }}$ : otherwise, there would be an edge in $\Gamma_{\text {out }}$, whose source is of the second type. Hence, since the polyvector fields $\alpha$ and $\beta$ are respectively associated to vertices in $A_{1} \sqcup B_{1}$ and $A_{2} \sqcup B_{2}$, then only copies of $Q$ can be associated to the vertices of $\Gamma_{\text {out }}$. Therefore the vertices of $\Gamma_{\text {out }}$ have all exactly one outgoing edge, and consequently $\Gamma_{\text {out }}$ can be only the trivial graph with no vertex of the first type and exactly two vertices of the second type. In other words, $\Gamma$ is the disjoint union $\Gamma_{\text {int }}^{1} \sqcup \Gamma_{\text {int }}^{2}$. Summarizing all these arguments, we get

$$
\mathcal{W}_{\Gamma}=\int_{\mathcal{Z}_{1} \cap \partial_{T} \bar{C}_{n+2, m}^{+}} \omega_{\Gamma}=\int_{\bar{C}_{A_{1}, B_{1}}^{+}} \omega_{\Gamma_{\mathrm{int}}^{1}} \int_{\bar{C}_{A_{2}, B_{2}}^{+}} \omega_{\Gamma_{\mathrm{int}}^{2}}=\mathcal{W}_{\Gamma_{\mathrm{int}}^{1}} \mathcal{W}_{\Gamma_{\mathrm{int}}^{2}} .
$$

For any other graph $\Gamma$, it follows from the previous arguments that $\mathcal{W}_{\Gamma}=0$.

Combining Lemma 7.6 with (7.11), we finally obtain

$$
\begin{aligned}
\mathcal{U}_{Q}(\alpha) \cup \mathcal{U}_{Q}(\beta) & =\sum_{k, l \geq 0} \frac{1}{k ! l !} \sum_{\substack{\Gamma_{1} \in \mathcal{G}_{k+1, m_{1}} \\
\Gamma_{2} \in \mathcal{G}_{l+1, m_{2}}}} \mathcal{W}_{\Gamma_{1} \sqcup \Gamma_{2}} B_{\Gamma_{1} \sqcup \Gamma_{2}}(\alpha, \beta, \underbrace{Q, \ldots, Q}_{k+l \text { times }}) \\
& =\sum_{n \geq 0} \frac{1}{n !} \sum_{\substack{\Gamma \in \mathcal{G}_{n+2, m} \\
\mathcal{W}_{\Gamma}^{1} B_{\Gamma}}}(\alpha, \beta, \underbrace{Q, \ldots, Q}_{n \text { times }}) .
\end{aligned}
$$

The combinatorial factor $\frac{1}{n !}$ appears, instead of $\frac{1}{k ! l !}$, as a consequence of the fact that the sum is over graphs which split into a disjoint union of two subgraphs, and we have to take care of the possible equivalent graphs splitting into the same disjoint union.

Remark 7.7. We could have chosen $y$ to be the unique point sitting in the other copy of $C_{0,2}^{+}$inside $\partial \bar{C}_{2,0}^{+}$, i.e. the one in which the vertex 2 is on the left of the vertex 1 . In this case Proposition 7.5 remains true if one replaces the l.h.s. of (7.10) by $\pm \mathcal{U}_{Q}(\beta) \cup \mathcal{U}_{Q}(\alpha)$. Since $\cup$ is known to be commutative on the level of cohomology, then the choice of the copy of $C_{0,2}^{+}$ is not really important. 


\section{The map $\mathcal{H}_{Q}$ AND the homotopy ARgument}

In this Section we define the weights $\widetilde{\mathcal{W}}_{\Gamma}$ appearing in the defining formula (5.3) for $\mathcal{H}_{Q}$ and prove that, together with $\mathcal{U}_{Q}$, it satisfies the homotopy equation (5.1). We continue to follow closely the treatment of Manchon and Torossian [26]. To evaluate certain integral weights, we again need the explicit description of boundary strata of codimension 1 of $\bar{C}_{n, m}^{+}$, for whose discussion we refer to the end of paragraph 6.2.

\subsection{The complete homotopy argument.}

We have proved in Subsection 7.2 and Subsection 7.3, that the expressions $\mathcal{U}_{Q}(\alpha \wedge \beta)$ and $\mathcal{U}_{Q}(\alpha) \cup \mathcal{U}_{Q}(\beta)$ can be rewritten by means of the integral weights over $\mathcal{Z}_{0}=F^{-1}(x)$ and $\mathcal{Z}_{1}=F^{-1}(y)$, where we recall that $F:=\pi_{\{1,2\}, \emptyset}: \bar{C}_{n+2, m}^{+} \rightarrow \bar{C}_{2,0}^{+}$, and $x \in C_{2} \subset \partial \bar{C}_{2,0}^{+}$and $y \in C_{0,2}^{+} \subset \partial \bar{C}_{2,0}^{+}$are arbitrary.

It is thus natural to consider a continuous path $\gamma:[0 ; 1] \rightarrow \bar{C}_{2,0}^{+}$such that $x:=\gamma(0) \in C_{2}$, $y:=\gamma(1) \in C_{0,2}^{+}$, and $\gamma(t) \in C_{2,0}^{+}$for any $\left.t \in\right] 0,1[$. We therefore define

$$
\mathcal{Z}:=F^{-1}(\gamma(] 0,1[)) \subset \bar{C}_{n+2, m}^{+} .
$$

Its closure $\overline{\mathcal{Z}}$ is the preimage of $\gamma([0,1])$ under the projection $F$. Then the boundary of $\overline{\mathcal{Z}}$ splits into the disjoint union

$$
\partial \overline{\mathcal{Z}}=\mathcal{Z}_{0} \sqcup \mathcal{Z}_{1} \sqcup\left(\mathcal{Z} \cap \partial \bar{C}_{n+2, m}^{+}\right) .
$$

The third boundary component will be denoted by $Y$.

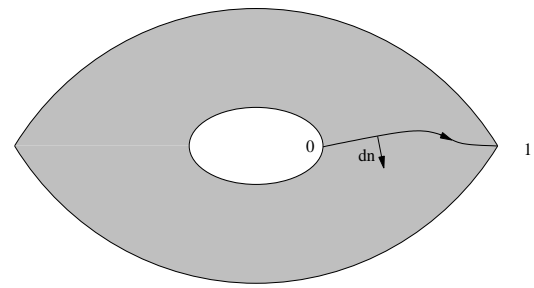

Figure 3 - the path $\gamma$ in Kontsevich's eye

Since, by assumption, $\gamma(] 0,1[)$ lies in the interior $C_{2,0}^{+} \subset \bar{C}_{2,0}^{+}$, then it follows that $Y$ intersects only the following five types of boundary strata of codimension 1 of $\bar{C}_{n+2, m}^{+}$:

i) there is a subset $A_{1}$ of $\{1, \ldots, n+2\}$, containing 1 , but not 2 , such that the points of the first type labelled by $A_{1}$ collapse together to a single point of the first type;

ii) there is a subset $A_{2}$ of $\{1, \ldots, n+2\}$, containing 2 , but not 1 , such that the points of the first type labelled by $A_{2}$ collapse together to a single point of the first type;

iii) there is a subset $A$ of $\{1, \ldots, n+2\}$, containing neither 1 nor 2 , such that the points of the first type labelled by $A$ collapse together to a single point of the first type;

$i v$ ) there is a subset $A$ of $\{1, \ldots, n+2\}$, containing neither 1 nor 2 , and an ordered subset $B$ of $\{1, \ldots, m\}$ of consecutive integers, such that the points labelled by $A$ (of the first type) and by $B$ (of the second type) collapse together to a single point of the second type;

$v$ ) there is a subset $A$ of $\{1, \ldots, n+2\}$, containing both 1 and 2 , and an ordered subset $B$ of $\{1, \ldots, m\}$ of consecutive integers, such that the points labelled by $A$ (of the first type) and by $B$ (of the second type) collapse together to a single point of the second type.

Remark 8.1. We observe that there is no intersection with a boundary stratum for which there is a subset $A$ of $\{1, \ldots, n+2\}$ such that the points labelled by $A$ collapse together to a single point of the first type. This is because such a boundary stratum (by the arguments of Proposition 7.2) intersects non-trivially $\mathcal{Z}_{0}$, and $Y, \mathcal{Z}_{0}$ and $\mathcal{Z}_{1}$ are pairwise disjoint. 
For a graph $\Gamma \in \mathcal{G}_{n+2, m}$ we define new weights

$$
\mathcal{W}_{\Gamma}^{2}=\int_{Y} \omega_{\Gamma}, \quad \text { and } \quad \widetilde{\mathcal{W}}_{\Gamma}=\int_{\mathcal{Z}} \omega_{\Gamma},
$$

with the same notations as in Definition 6.8 of Subsection 6.3. Stokes' Theorem implies

$$
\int_{\partial \overline{\mathcal{Z}}} \omega_{\Gamma}=\int_{\mathcal{Z}} \mathrm{d} \omega_{\Gamma}=0
$$

Using the orientation choices for $\mathcal{Z}$, for which we refer to [26], together with (8.1), the previous identity implies the relation

$$
\mathcal{W}_{\Gamma}^{0}=\mathcal{W}_{\Gamma}^{1}+\mathcal{W}_{\Gamma}^{2}
$$

Using Proposition 7.2, Proposition 7.5 and the above identity involving Stokes' Theorem, we obtain that the l.h.s. of the homotopy equation (5.1) equals

$$
-\sum_{n \geq 0} \frac{1}{n !} \sum_{\Gamma \in \mathcal{G}_{n+2, m}} \mathcal{W}_{\Gamma}^{2} B_{\Gamma}(\alpha, \beta, \underbrace{Q, \ldots, Q}_{n \text { times }}) .
$$

Hence, to prove that $\mathcal{H}_{Q}$, given by (5.3), satisfies (5.1) together with $\mathcal{U}_{Q}$, it remains to show that for fixed $n$ and $m$, the following identity holds true:

$$
\begin{aligned}
& \sum_{\Gamma \in \mathcal{G}_{n+2, m}} \mathcal{W}_{\Gamma}^{2} B_{\Gamma}(\alpha, \beta, \underbrace{Q, \ldots, Q}_{n \text { times }})=-\sum_{\Gamma \in \mathcal{G}_{n+2, m}} \widetilde{\mathcal{W}}_{\Gamma} \mathrm{d}_{H}\left(B_{\Gamma}(\alpha, \beta, \underbrace{Q, \ldots, Q}_{n \text { times }})\right) \\
& -n\left(\sum_{\Gamma \in \mathcal{G}_{n+1, m}} \widetilde{\mathcal{W}}_{\Gamma} Q \cdot\left(B_{\Gamma}(\alpha, \beta, \underbrace{Q, \ldots, Q}_{n-1 \text { times }})\right)+\sum_{\Gamma \in \mathcal{G}_{n+1, m}} \widetilde{\mathcal{W}}_{\Gamma}\left(B_{\Gamma}(Q \cdot \alpha, \beta, \underbrace{Q, \ldots, Q}_{n-1 \text { times }})\right)\right. \\
& \left.+(-1)^{\|\alpha\|} \sum_{\Gamma \in \mathcal{G}_{n+1, m}} \widetilde{\mathcal{W}}_{\Gamma}\left(B_{\Gamma}(\alpha, Q \cdot \beta, \underbrace{Q, \ldots, Q}_{n-1 \text { times }})\right)\right) .
\end{aligned}
$$

In the forthcoming Subsection 8.2 we sketch the proof of Identity (8.2). For a more detailed treating of signs appearing in the forthcoming arguments, we refer to [26].

Summarizing, the sum of (8.3) and (8.4) from paragraph 8.2.1, and of (8.6) from paragraph 8.2.2, we get the term in (8.2) involving the Hochschild differential of (5.2). The sum of (8.5) from paragraph 8.2.1 and of (8.7) from paragraph 8.2.2 yields the term with the action of the cohomological vector field $Q$ on $D_{\text {poly }} V$. In paragraph 8.2.3 one obtains the vanishing of terms which contain the action of $Q$ on itself. Finally, (8.8) and (8.9) from paragraph 8.2.4 yield the remaining terms in (8.2). Thus, we have proved (5.1).

\subsection{Contribution to $\mathcal{W}_{\Gamma}^{2}$ of boundary components in $Y$.}

The discussion is analogous to the one sketched in the proof of Proposition 7.1.

\subsubsection{Boundary strata of type $v)$.}

We consider a boundary stratum $C$ of $Y$ of type $v$ ): there exists a subset $A$ of $\{1, \ldots, n+2\}$ and an ordered subset $B$ of $\{1, \ldots, m\}$ of consecutive integers, such that

$$
C=\mathcal{Z} \cap\left(\bar{C}_{A, B}^{+} \times \bar{C}_{n-|A|+2, m-|B|+1}^{+}\right) .
$$

Accordingly, by means of Fubini's Theorem, the integral weight of a graph $\Gamma \in \mathcal{G}_{n+2, m}$, restricted to $C$, can be rewritten as

$$
\mathcal{W}_{\left.\Gamma\right|_{C}}=\int_{\partial_{C} \overline{\mathcal{Z}}} \omega_{\Gamma}=\int_{\mathcal{Z} \cap \bar{C}_{A, B}^{+}} \omega_{\Gamma_{\mathrm{int}}} \int_{\bar{C}_{n-|A|+2, m-|B|+1}^{+}} \omega_{\Gamma_{\mathrm{out}}}
$$


Here we have used the same improper notation as in the proof of Lemma 7.3, and, as usual, $\Gamma_{\text {int }}\left(\right.$ resp. $\Gamma_{\text {out }}$ ) denotes the subgraph of $\Gamma$ whose vertices are labelled by $A \sqcup B$ (resp. the subgraph obtained by contracting $\Gamma_{\text {int }}$ to a single point of the second type).

The polyvector fields $\alpha$ and $\beta$ have been put on the vertices labelled by 1 and 2 , which belong to $A$ : hence, only copies of the cohomological vector field $Q$ can be put on the first type vertices of $\Gamma_{\text {out }}$. In other words, first type vertices of $\Gamma_{\text {out }}$ have a single outgoing edge. Then, for the same combinatorial reason as in the proof of Proposition $7.1 \Gamma_{\text {out }}$ is
either
i)

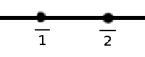
or
ii)

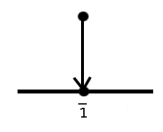

In both cases, the integral weight corresponding to $\Gamma_{\text {out }}$ is normalized, up to some signs coming from orientation choices (which we will again neglect, as before).

The directed subgraph $\Gamma_{\text {int }}$ belongs obviously to $\mathcal{G}_{n+2, m-1}$, resp. $\mathcal{G}_{n+1, m}$, since in case $i),|A|=n+2$ and $|B|=m-1$, whereas, in case $i i),|A|=n+1$ and $|B|=m$. Case $i$ ), furthermore, includes two subcases, namely, since $|B|=m-1$, and since $B$ consists only of consecutive integers, it follows immediately that $B=\{\overline{1}, \ldots, \overline{m-1}\}$ or $B=\{\overline{2}, \ldots, \bar{m}\}$. From the point of view of polydifferential operators, the graph $\Gamma_{\text {out }}$ corresponds, in both subcases of $i$ ), to the multiplication operator, whereas, in case $i i$ ), it corresponds to the action of the cohomological vector field $Q$, placed on the vertex of the first type, on a function on $V$, placed on the vertex of the second type.

All these arguments yield the following expressions for the contributions to the left handside of (8.2) coming from boundary strata of type $v$ ):

$$
\begin{gathered}
\sum_{\Gamma \in \mathcal{G}_{n+2, m-1}} \pm \widetilde{\mathcal{W}}_{\Gamma} f_{1}\left(B_{\Gamma}(\alpha, \beta, \underbrace{Q, \ldots, Q}_{n \text { times }})\left(f_{2}, \ldots, f_{m}\right)\right), \\
\sum_{\Gamma \in \mathcal{G}_{n+2, m-1}} \pm \widetilde{\mathcal{W}}_{\Gamma}\left(B_{\Gamma}(\alpha, \beta, \underbrace{Q, \ldots, Q}_{n \text { times }})\left(f_{2}, \ldots, f_{m}\right)\right) f_{m}, \\
\sum_{\Gamma \in \mathcal{G}_{n+1, m}} \pm \widetilde{\mathcal{W}}_{\Gamma} Q \cdot\left(B_{\Gamma}(\alpha, \beta, \underbrace{Q, \ldots, Q}_{n-1 \text { times }})\left(f_{1}, \ldots, f_{m}\right)\right) .
\end{gathered}
$$

\subsubsection{Boundary strata of type iv).}

We consider now a boundary stratum $C$ of $Y$ of the fourth type: in this case, there exists a subset $A$ of $\{1, \ldots, n+2\}$, containing neither the vertex labelled by 1 nor by 2 , and an ordered subset $B$ of $\{1, \ldots, m\}$, such that

$$
C=\mathcal{Z} \cap\left(\bar{C}_{A, B}^{+} \times \bar{C}_{n-|A|+2, m-|B|+1}^{+}\right) .
$$

One more, Fubini's Theorem implies the factorization

$$
\mathcal{W}_{\left.\Gamma\right|_{C}}=\int_{C} \omega_{\Gamma}=\int \omega_{\Gamma_{\text {int }}} \int \omega_{\Gamma_{\text {out }}^{+}, B} \quad{\mathcal{Z} \cap \bar{C}_{n-|A|+2, m-|B|+1}^{+}} .
$$

The vertices labelled by 1 and 2, to which we have put the polyvector vector fields $\alpha$ and $\beta$, are vertices of the graph $\Gamma_{\text {out }}$ : hence, every first type vertex of $\Gamma_{\text {int }}$ has exactly one outgoing edge. Again, as in the proof of Proposition 7.1 and thanks to the vanishing Lemma $6.9, \Gamma_{\text {int }}$ can be only of the following two types:

i)

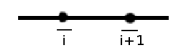

and ii)

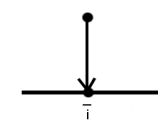


In case $i$ ), resp. $i i), \Gamma_{\text {out }}$ is a graph in $\mathcal{G}_{n+2, m-1}$, resp. in $\mathcal{G}_{n+1, m}$; in case $\left.i\right), A=\emptyset$ and $B=\{\bar{i}, \bar{i}+1\}$ (since points of the second type are ordered), for $\bar{i}=\overline{1}, \ldots, \bar{m}$, while, in case ii), $A=\{i\}$ and $B=\{\bar{j}\}$, for $i=1, \ldots, n+2$ and $\bar{j}=\overline{1}, \ldots, \bar{m}$.

Up to signs arising from orientation choices, which we have neglected so far, both integrals corresponding to $i$ ) and $i i$ ) are normalized. The graph $\Gamma_{\text {int }}$ corresponds, in terms of the polydifferential operators $B_{\Gamma}$, to the product of two functions on $V$, which have been put to the vertices labelled by $\bar{i}$ and $\overline{i+1}$, in case $i$ ); on the other hand, in case $i i)$, the graph $\Gamma_{\text {int }}$ corresponds to the situation, where the cohomological vector fields $Q$ acts, as a derivation, on a function on $V$, which has been put on the vertex $\bar{j}$.

Using all previous arguments, we obtain the following two expressions for the contributions to the left hand-side of (8.2) coming from boundary strata of type $i v)$ :

$$
\begin{aligned}
& \sum_{i=1}^{m-1} \sum_{\Gamma \in \mathcal{G}_{n+2, m-1}} \pm \widetilde{\mathcal{W}}_{\Gamma} B_{\Gamma}(\alpha, \beta, \underbrace{Q, \ldots, Q}_{n \text { times }})\left(f_{1}, \ldots, f_{i} f_{i+1}, \ldots, f_{m}\right), \\
& \sum_{i=1}^{m} \sum_{\Gamma \in \mathcal{G}_{n+1, m}} \pm \widetilde{\mathcal{W}}_{\Gamma} B_{\Gamma}(\alpha, \beta, \underbrace{Q, \ldots, Q}_{n-1 \text { times }})\left(f_{1}, \ldots, Q \cdot f_{i}, \ldots, f_{m}\right),
\end{aligned}
$$

for any collection $\left\{f_{1}, \ldots, f_{m}\right\}$ of $m$ functions on $V$.

\subsubsection{Boundary strata of type iii).}

We examine a boundary stratum $C$ of $Y$ of the third type, thus, there is a subset $A$ of $\{1, \ldots, n+2$, containing neither the vertex labelled by 1 nor by 2 , such that

$$
C=\mathcal{Z} \cap\left(\bar{C}_{A} \times \bar{C}_{n-|A|+3, m}^{+}\right) .
$$

The contribution coming from $C$ to the integral weight is, again by means of Fubini's Theorem,

$$
\mathcal{W}_{\left.\Gamma\right|_{C}}=\int_{C} \omega_{\Gamma}=\int_{\bar{C}_{A}} \omega_{\Gamma_{\mathrm{int}}} \int_{\mathcal{Z} \cap \bar{C}_{n-|A|+3, m}^{+}} \omega_{\Gamma_{\mathrm{out}}} .
$$

Since the polyvector fields $\alpha$ and $\beta$ have been put on the vertices labelled by 1 and 2 , which do not belong to $A$, it follows that only copies of $Q$ have been put on the vertices of $\Gamma_{\text {int }}$. We focus in particular on the integral contributions coming from $\Gamma_{\text {int }}$ : by Lemma 6.10 , if $|A| \geq 3$, such contributions vanish, whence we are left with only one possible directed subgraph $\Gamma_{\text {int }}$, namely $\Gamma_{\text {int }}$ consists of exactly two vertices of the first type joined by exactly one edge. The corresponding weight is normalized, by the properties of the angle function. The graph $\Gamma_{\text {out }}$ is easily verified to be in $\mathcal{G}_{n+1, m}$; the polydifferential operator corresponding to $\Gamma_{\text {int }}$ represents the adjoint action of $Q$ on itself, by its very construction. Since $Q$ is, by assumption, a cohomological vector field, it follows that such a contribution vanishes by the property $[Q, Q]=\frac{1}{2} Q \circ Q=0$. It thus follows that boundary strata of type $\left.i i i\right)$ do not contribute to the left hand-side of (8.2).

\subsubsection{Boundary strata of type $i$ ) and $i i)$.}

We consider a boundary stratum $C$ of $Y$ of type $i$ ). By its very definition, for such a stratum $C$ there exists a subset $A_{1}$ of $\{1, \ldots, n+2\}$, containing the vertex labelled by 1 , but not the vertex labelled by 2 , such that

$$
C=\mathcal{Z} \cap\left(\bar{C}_{A_{1}} \times \bar{C}_{n-|A|+3, m}^{+}\right) .
$$

By means of Fubini's Theorem, we obtain the following factorization for the integral weight $\mathcal{W}_{\Gamma}$, when restricted to $C$,

$$
\mathcal{W}_{\left.\Gamma\right|_{C}}=\int_{C} \omega_{\Gamma}=\int_{\bar{C}_{A_{1}}} \omega_{\Gamma_{\mathrm{int}}} \int_{\mathcal{Z} \cap \bar{C}_{n-|A|+3, m}^{+}} \omega_{\Gamma_{\mathrm{out}}} .
$$


We focus our attention on the integral contribution coming from $\Gamma_{\text {int }}$ : as in Subsubsection 8.2 .3 , by means of Lemma 6.10 , the only possible subgraph $\Gamma_{\text {int }}$ yielding a non-trivial integral contribution is the graph consisting of two vertices of the first type joined by exactly one edge, in which case the contribution is normalized (up to some signs, coming from orientation choices, which we neglect, as we have done before). By assumption, one of the two vertices is labelled by 1 and the other one is labelled by an $i=1, \ldots, n+2, i \neq 2$ : there are hence two possible graphs, namely, $i$ ) when the edge has, as target, the vertex labelled by 1 , and $i$ ) when the edge has, as source, the vertex labelled by 1 . Since the remaining vertex is not labelled by 2 , in terms of polydifferential operators, we have two situations: a copy of $Q$ acts, as a differential operator of order 1, on the components of the polyvector field $\alpha$, in case $i$ ), or one of the derivations of the polyvector field $\alpha$ acts, as a differential operator of order 1 , on the components of $Q$, in case $i i$ ). Finally, the graph $\Gamma_{\text {out }}$ belongs obviously to $\mathcal{G}_{n+1, m}$.

By the previous arguments, and by the very definition of the Lie $\mathfrak{X}_{V}$-module structure on polyvector fields, the contributions to the left hand-side of (8.2) coming from boundary strata of type $i$ ) can be written as

$$
\sum_{\Gamma \in \mathcal{G}_{n+1, m}} \pm \widetilde{\mathcal{W}}_{\Gamma} B_{\Gamma}(Q \cdot \alpha, \beta, \underbrace{Q, \ldots, Q}_{n-1 \text { times }}) .
$$

As for boundary strata of $Y$ of type $i i$ ), we may repeat almost verbatim the previous arguments, the only difference in the final result being that the rôle played by the polyvector field $\alpha$ will be now played by $\beta$, hence the contributions to the left hand-side of $(8.2)$ coming from boundary strata of type $i i$ ) are exactly

$$
\sum_{\Gamma \in \mathcal{G}_{n+1, m}} \pm \widetilde{\mathcal{W}}_{\Gamma} B_{\Gamma}(\alpha, Q \cdot \beta, \underbrace{Q, \ldots, Q}_{n-1 \text { times }}) .
$$

\subsection{Twisting by a supercommutative DG algebra.}

We consider finally a supercommutative DG algebra $\left(\mathfrak{m}, \mathrm{d}_{\mathfrak{m}}\right)$ : typically, instead of considering $T_{\text {poly }} V$ and $D_{\text {poly }} V$, for a superspace $V$ as before, we consider their twists w.r.t. $\mathfrak{m}$ :

$$
T_{\text {poly }}^{\mathfrak{m}} V:=T_{\text {poly }} V \otimes \mathfrak{m} \quad \text { and } \quad D_{\text {poly }}^{\mathfrak{m}} V:=D_{\text {poly }} V \otimes \mathfrak{m} .
$$

Since $\mathfrak{m}$ is supercommutative, the Lie bracket on $\mathfrak{X}_{V}$ determines a graded Lie algebra structure on $\mathfrak{X}_{V}^{\mathfrak{m}}:=\mathfrak{X}_{V} \otimes \mathfrak{m}$ :

$$
[v \otimes \mu, w \otimes \nu]=(-1)^{|w||\mu|}[v, w] \otimes \mu \nu .
$$

Hence, for any choice of a supercommutative DG algebra $\left(\mathfrak{m}, \mathrm{d}_{\mathfrak{m}}\right)$, there are two graded Lie $\mathfrak{X}_{V}^{\mathfrak{m}}$-modules $T_{\text {poly }}^{\mathfrak{m}} V$ and $D_{\text {poly }}^{\mathfrak{m}} V$. Moreover the differential $\mathrm{d}_{\mathfrak{m}}$ extends naturally to a differential on $T_{\text {poly }}^{\mathfrak{m}} V$ and $D_{\text {poly }}^{\mathfrak{m}} V$. It is easy to verify that the differential $\mathrm{d}_{\mathfrak{m}}$ (super)commutes with the Hochschild differential $d_{\mathrm{H}}$ on $D_{\text {poly }}^{\mathfrak{m}} V$.

We now consider an $\mathfrak{m}$-valued vector field $Q \in \mathfrak{X}_{V}^{\mathfrak{m}}$ of degree 1 which additionally satisfies the so-called Maurer-Cartan equation

$$
\mathrm{d}_{\mathfrak{m}} Q+\frac{1}{2}[Q, Q]=\mathrm{d}_{\mathfrak{m}} Q+Q \circ Q=0 .
$$

We observe that, if $\mathfrak{m}=k$ (with $k$ placed in degree 0 ) then $Q$ is simply a cohomological vector field on $V$ as in Definition 5.2. The Maurer-Cartan equation implies that $\mathrm{d}_{\mathfrak{m}}+Q \cdot$ is a linear operator of (total) degree 1 on $T_{\text {poly }}^{\mathfrak{m}} V$, which additionally squares to 0 ; moreover, the product $\wedge$ on $T_{\text {poly }} V$ extends naturally to a supercommutative graded associative product $\wedge$ on $T_{\text {poly }}^{\mathfrak{m}} V$, and $\mathrm{d}_{\mathfrak{m}}+Q$. is obviously a degree one derivation of this product. Therefore,

$$
\left(T_{\text {poly }}^{\mathfrak{m}} V, \wedge, \mathrm{d}_{\mathfrak{m}}+Q \cdot\right)
$$

is a DG algebra. 
One obtains in exactly the same way a DG algebra

$$
\left(D_{\text {poly }}^{\mathfrak{m}} V, \cup, d_{H}+\mathrm{d}_{\mathfrak{m}}+Q \cdot\right) .
$$

Theorem 5.3 can be generalized to these DG algebras as follows.

Theorem 8.2. For any degree one solution $Q \in \mathfrak{X}_{V}^{\mathfrak{m}}$ of the Maurer-Cartan equation, the $\mathfrak{m}$-linear map $\mathcal{U}_{Q}$ given by (5.2) defines a morphism of complexes

$$
\left(T_{\text {poly }}^{\mathfrak{m}} V, \wedge, \mathrm{d}_{\mathfrak{m}}+Q \cdot\right) \stackrel{\mathcal{U}_{Q}}{\longrightarrow}\left(D_{\text {poly }}^{\mathfrak{m}} V, \cup, d_{\mathrm{H}}+\mathrm{d}_{\mathfrak{m}}+Q \cdot\right)
$$

which induces an isomorphism of (graded) algebras on the corresponding cohomologies.

Proof. The proof follows along the same lines as the proof of Theorem 5.3, which can be repeated almost verbatim. The differences arises when discussing

- the morphism property (7.1) for $\mathcal{U}_{Q}$,

- the homotopy property (5.1) for $\mathcal{U}_{Q}$ and $\mathcal{H}_{Q}$.

In both cases one must replace $\left(d_{H}+Q \cdot\right)$ where it appears in the equation by $\left(d_{H}+\mathrm{d}_{\mathfrak{m}}+Q \cdot\right)$.

For the homotopy property (5.1), the core of the proof lies in the discussion of the boundary strata for the configuration spaces appearing in (8.2): the relevant boundary strata in the present proof are those of Subsubsection 8.2.3. We can repeat the same arguments in the discussion of the corresponding integral weights: using the very same notations as in Subsubsection 8.2.3, the polydifferential operator corresponding to $\Gamma_{\text {int }}$ is one half times the adjoint action of $Q$ on itself, which, in this case, does not square to 0, but equals (up to $\operatorname{sign}) \mathrm{d}_{\mathfrak{m}} Q$ by the Maurer-Cartan equation. Using the graded Leibniz rule for $\mathrm{d}_{\mathfrak{m}}$, we get all homotopy terms which contain $d_{\mathfrak{m}}$. The discussion of the remaining boundary strata remains unaltered.

The very same argument also works for the morphism property (7.1). Nevertheless, we see in the next Section that (7.1) can be obtained as a consequence of the explicit form of $\mathcal{U}_{Q}$, avoiding the discussion on possible contributions of the boundary components in the proof of Proposition 7.1. 


\section{The explicit FORM of $\mathcal{U}_{Q}$}

In this Section we compute explicitly the quasi-isomorphism $\mathcal{U}_{Q}(5.2)$, following closely [6, Section 8]. Namely, we first argue about the possible shapes of the graphs $\Gamma$ involved in the construction of $\mathcal{U}_{Q}$ : by the way, this was already done, although not as precisely as in the present Section, in the proof of Proposition 7.5.

\subsection{Graphs contributing to $\mathcal{U}_{Q}$.}

We now recall that, in (5.2), we need a polyvector field $\alpha$ on the superspace $V$ and a cohomological vector field $Q$. We consider a graph $\Gamma \in \mathcal{G}_{n+1, m}$, appearing in (5.2): on one of its vertices of the first type, we put $\alpha$, while, on the remaining $n$ vertices of the first type we put copies of $Q$. Since $Q$ is a vector field, in particular, from any edge, where $Q$ has been put, departs exactly one edge. Additionally, Lemma 6.9 from Subsection 6.3 implies that $\Gamma$ cannot contain bivalent vertices of the first type with exactly one ingoing and exactly one outgoing edge: therefore, a given vertex of the first type, where $Q$ has been put, has only one outgoing edge and at least two ingoing edges. In fact, this result implies that any such vertex has exactly two ingoing edges: one coming from another vertex of the first type, where $Q$ has been put, and the other one coming from the vertex of the first type, where $\alpha$ has been put.

Summarizing this argument, a general graph $\Gamma \in \mathcal{G}_{n+1, m}$, contributing (possibly) nontrivially to (5.2), is a wheeled tree, i.e. there is a chosen vertex $c$ of the first type, and a partition of $\{1, \ldots, n\}$ into $k$ disjoint subsets, such that from $c$ departs $m$ edges, joining $c$ to the $m$ vertices of the second type of $\Gamma$, and such that to $c$ are attached, by means of outgoing directed edges, $k$ wheels, the $i$-th wheel having exactly $l_{i}$ vertices (of the first type).

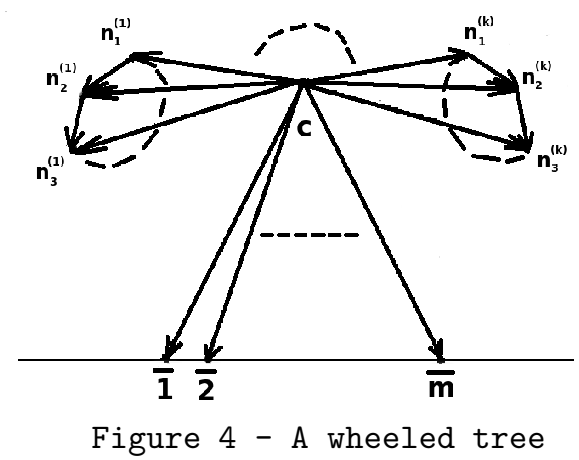

For a wheeled tree $\Gamma$ in $\mathcal{G}_{n+1, m}$, associated to $k$ wheels, whose length is $l_{i}, i=1, \ldots, k$, and $\sum_{i=1}^{k} l_{i}=n$, we denote by $\Sigma_{l_{i}}, i=1, \ldots, k$, resp. $A_{m}$, the $i$-th wheel with $l_{i}$ vertices, resp. the graph with exactly one vertex of the first type and $m$ vertices of the second type, and $m$ edges, whose directions and targets are obvious.
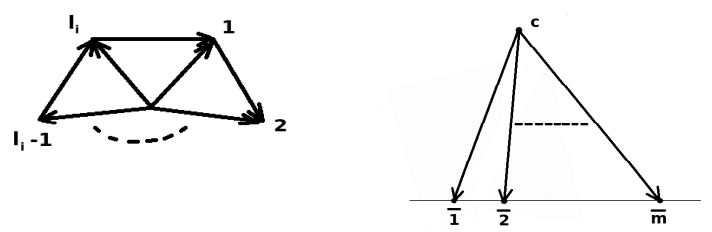

Figure 5 - The wheel $\Sigma_{l_{i}}$ (left) and the graph $A_{m}$ (right)

Lemma 9.1. For any positive integer $m \geq 1$, the identity holds true

$$
\mathcal{W}_{A_{m}}=\frac{1}{m !} \text {. }
$$


Sketch of the proof. The configuration space corresponding to the graph $A_{m}$ is $C_{1, m}^{+}$: by means of the action of $G_{2}$, we may e.g. put the only point of first type to i, while the remaining points of second type remain ordered, and are free to move on the real axis $\mathbb{R}$. Thus, $\bar{C}_{1, m}^{+}$corresponds to the open infinite $m$-simplex, consisting of $m$-tuples of points $\left(q_{1}, \ldots, q_{m}\right)$ satisfying

$$
-\infty<q_{1}<\cdots<q_{m}<\infty .
$$

On the other hand, the angle function, computed at $(\mathrm{i}, q), q$ in $\mathbb{R}$, is easily verified to be equal to

$$
\frac{\varphi(\mathrm{i}, q)}{2 \pi}=\frac{1}{2 \pi} \arg \left(\frac{q-\mathrm{i}}{q+\mathrm{i}}\right)=-\frac{1}{\pi} \arctan \left(\frac{1}{q}\right),
$$

up to some constant angle. Hence, the explicit expression for $\mathcal{W}_{A_{m}}$ is

$$
\mathcal{W}_{A_{m}}=\pi^{m} \int_{-\infty<q_{1}<\cdots<q_{m}<\infty} \frac{2 \mathrm{~d} q_{1}}{1+q_{1}^{2}} \wedge \cdots \wedge \frac{2 \mathrm{~d} q_{m}}{1+q_{m}^{2}} .
$$

Rewriting the previous expression as a multiple integral over the open infinite $m$-simplex, we may perform coordinate transformations on the integrand, which map the open infinite simplex to the open standard $m$-simplex: such coordinate transformations map the integrand function to 1 , thus we are left with the volume of the open standard $m$-simplex, which is well-known to be $\frac{1}{m !}$, whence the claim.

Lemma 9.2. If $l$ is an odd integer, then $\mathcal{W}_{\Sigma_{l}}$ vanishes.

Sketch of the proof. All vertices of the wheel $\Sigma_{l}$ are of the first type: the corresponding configuration space is $C_{l, 0}^{+}$. The action of $G_{2}$ permits to fix, as in the proof of Lemma 9.1, the central vertex of the wheel to i: hence, $C_{l, 0}^{+}$corresponds to the configuration space of $l-1$ points of the first type, which do not coincide with i. Then, the involution $z \mapsto-\bar{z}$ extends to an involution of $C_{l, 0}^{+}$, which changes the sign of the integrand and preserves the orientation of $C_{l, 0}^{+}$, since $l-1$ is even.

\section{2. $\mathcal{U}_{Q}$ as a contraction.}

By Lemma 9.2 we are concerned only with wheeled trees whose wheels have an even number of vertices. In order to compute explicitly the weight of such a wheeled tree $\Gamma$ in $\mathcal{G}_{n+1, m}$, we use the action of $G_{2}$ on $C_{n+1, m}^{+}$to put the central vertex of $\Gamma$ in i, similarly to what was done in Lemma 9.2. Denoting by $\bar{C}$ the compactification of $C_{n+1, m}^{+}$, where one point of the first type has been put in i, the weight of $\Gamma$ can be rewritten as

$$
\mathcal{W}_{\Gamma}=\int_{\bar{C}}\left(\bigwedge_{i=1}^{n} \mathrm{~d} \varphi_{g_{i}}\right) \wedge\left(\bigwedge_{j=1}^{n} \mathrm{~d} \varphi_{e_{i}}\right) \wedge\left(\bigwedge_{k=1}^{m} \mathrm{~d} \varphi_{f_{i}}\right)
$$

where the big wedge products are ordered according to the indices, i.e.

$$
\bigwedge_{i=1}^{n} \mathrm{~d} \varphi_{g_{i}}=\mathrm{d} \varphi_{g_{1}} \wedge \cdots \wedge \mathrm{d} \varphi_{g_{n}}
$$

and so on. Further, the notations are as follows: $g_{i}$, resp. $e_{j}$, resp. $f_{k}$, denotes the only edge outgoing from the $i$-th vertex of the first type (where the vertex labelled by $i$ does not coincide with the central vertex $c$ ), resp. the edge connecting the central vertex $c$ to the $j$-th vertex of the first type, resp. the edge connecting the central vertex $c$ to the $k$-th vertex of the second type.

At this point, we may use the fact that there is an action of the permutation group $\mathfrak{S}_{n} \subset \mathfrak{S}_{n+1}$ on $\bar{C}_{n+1, m}^{+}$, where $\mathfrak{S}_{n}$ contains all permutations which keep the point of the 
first type corresponding to the central vertex $c$ of $\Gamma$ fixed. We choose a permutation $\sigma$ in such a way that the weight of $\Gamma$ takes the form

$$
\mathcal{W}_{\Gamma}=\int_{\bar{C}}\left(\bigwedge_{i=1}^{n} \mathrm{~d} \varphi_{g_{\sigma(i)}}\right) \wedge\left(\bigwedge_{j=1}^{n} \mathrm{~d} \varphi_{e_{\sigma(i)}}\right) \wedge\left(\bigwedge_{k=1}^{m} \mathrm{~d} \varphi_{p_{i}}\right)
$$

The permutation $\sigma$ is chosen so that for each wheel $\Sigma_{l}$ of $\Gamma$ the $i$-th vertex of $\Sigma_{l}$ has the only outgoing edge $g_{\sigma(i)}$ and the two incoming edges $g_{\sigma(i-1)}$ (modulo the length of the wheel) and $e_{\sigma(i)}$. After reordering of the differential forms, the weight of $\Gamma$ can be finally rewritten as

$$
\begin{aligned}
\mathcal{W}_{\Gamma}=(-1)^{1 \leq p<q \leq k} & \int_{\bar{C}} l_{p} l_{q} \\
\cdots & \wedge\left(\bigwedge_{i_{1}=1}^{l_{1}} \mathrm{~d} \varphi_{g_{\sigma\left(i_{1}\right)}} \wedge \bigwedge_{j_{1}=1}^{l_{1}} \mathrm{~d} \varphi_{g_{\sigma\left(j_{1}\right)}}\right) \wedge \cdots \\
\cdots & \left.\mathrm{d} \varphi_{g_{\sigma\left(i_{k}\right)}} \wedge \bigwedge_{i_{k}=l_{k-1}+1}^{l_{k}} \mathrm{~d} \varphi_{\left.g_{\sigma\left(j_{k}\right)}\right)}\right) \wedge\left(\bigwedge_{k=1}^{m} \mathrm{~d} \varphi_{p_{i}}\right)
\end{aligned}
$$

where, again, the ordering of the 1 -forms in the big wedge products are w.r.t. the natural ordering of the indices. In $(9.1), l_{i}, i=1, \ldots, k$, denotes the length of the $i$-th wheel. The sign in front of the integral comes from the reordering of the wheels. The integrand in (9.1) is the product of the integrands corresponding to the wheels of $\Gamma$ and to $A_{m}$ : Fubini's Theorem (together with Lemma 9.1) implies then the following factorization of the weight of $\Gamma$,

$$
\mathcal{W}_{\Gamma}=(-1)^{1 \leq p<q \leq k} l_{p} \frac{\mathcal{W}_{\Sigma_{l_{1}}} \cdots \mathcal{W}_{\Sigma_{l_{k}}}}{m !}
$$

Using the same notations for the edges of a wheeled tree $\Gamma$ as in (9.1), the polydifferential operator corresponding to $\Gamma$ takes the explicit form

$$
\begin{aligned}
& B_{\Gamma}(\alpha, \underbrace{Q, \ldots, Q}_{n \text { times }})\left(f_{1}, \ldots, f_{m}\right)= \\
& =\alpha^{p_{1}, \ldots, p_{n}, q_{1}, \ldots, q_{m}}\left(\partial_{p_{\sigma(1)}} \partial_{r_{\sigma\left(l_{1}\right)}} Q^{r_{\sigma(1)}} \partial_{p_{\sigma(2)}} \partial_{r_{\sigma(1)}} Q^{r_{\sigma(2)}} \cdots \partial_{p_{\sigma\left(l_{1}\right)}} \partial_{r_{\sigma\left(l_{1}-1\right)}} Q^{r_{\sigma\left(l_{1}\right)}}\right) \cdots \\
& \cdots\left(\partial_{q_{1}}\left(f_{1}\right) \cdots \partial_{q_{m}}\left(f_{m}\right)\right),
\end{aligned}
$$

where the product is over all wheels of $\Gamma$, and $\sigma$ is the same permutation as before, needed to reorganize the orderings of the wheels. In order to simplify notations in the previous formula, we introduce the supermatrix-valued 1-form $\Xi \in \Omega^{1}(V) \otimes \operatorname{End}(V[1])$, which is explicitly given by the formula

$$
\Xi_{i}^{j}=\mathrm{d}\left(\partial_{i} Q^{j}\right)=\partial_{k} \partial_{i} Q_{j} \mathrm{~d} x^{k},
$$

using (global) supercoordinates $\left\{x^{i}\right\}$ on $V$.

Then using (9.3) and the supertrace of endomorphisms of a finite-dimensional supervector space we have the following identity:

$$
B_{\Gamma}(\alpha, \underbrace{Q, \ldots, Q}_{n \text { times }})\left(f_{1}, \ldots, f_{m}\right)=\left\langle\alpha ; \operatorname{str}\left(\Xi^{l_{1}}\right) \wedge \cdots \wedge \operatorname{str}\left(\Xi^{l_{k}}\right) \wedge \mathrm{d} f_{1} \wedge \cdots \wedge \mathrm{d} f_{m}\right\rangle,
$$

where $\langle-;-\rangle$ denotes the pairing between differential forms and polyvector fields on $V$. The product between supermatrix-valued differential forms is the wedge product on the form part and multiplication of supermatrices for the supermatrix-part: then, $\operatorname{str}\left(\Xi^{l}\right)$ is an $l$-form on $V$. Using the contraction $\iota$ of polyvector fields w.r.t. differential forms and recalling that contraction is adjoint to wedge multiplication w.r.t. the pairing $\langle-;-\rangle$, the expression on 
the right hand-side of the previous identity is (neglecting, as before, any sign contribution)

$$
\begin{aligned}
& \left\langle\alpha, \operatorname{str}\left(\Xi^{l_{1}}\right) \wedge \cdots \wedge \operatorname{str}\left(\Xi^{l_{k}}\right) \wedge \mathrm{d} f_{1} \wedge \cdots \wedge \mathrm{d} f_{m}\right\rangle \\
= & \left\langle\iota_{\operatorname{str}\left(\Xi^{l_{1}}\right) \wedge \cdots \wedge \operatorname{str}\left(\Xi^{l_{k}}\right)} \alpha ;, \mathrm{d} f_{1} \wedge \cdots \wedge \mathrm{d} f_{m}\right\rangle \\
= & m !\left(I_{H K R}\left(\iota_{\operatorname{str}\left(\Xi^{l_{1}}\right) \wedge \cdots \wedge \operatorname{str}\left(\Xi^{l_{k}}\right)} \alpha\right)\right)\left(f_{1}, \ldots, f_{m}\right) .
\end{aligned}
$$

Hence, for a wheeled tree $\Gamma$ in $\mathcal{G}_{n+1, m}$ as before, using (9.2) for the weight $\mathcal{W}_{\Gamma}$ and (9.4) for the polydifferential operator $B_{\Gamma}$, we get the following simpler expression

$$
\mathcal{W}_{\Gamma} B_{\Gamma}(\alpha, \underbrace{Q, \ldots, Q}_{n \text { times }})=I_{H K R}\left(\left(X_{l_{k}} \cdots X_{l_{1}}\right) \alpha\right),
$$

where we have set

$$
X_{l_{i}}:=\mathcal{W}_{\Sigma_{l_{i}}} l_{\operatorname{str}\left(\Xi^{l_{i}}\right)} .
$$

In order to compute an explicit expression for $(5.2)$, we have to sum over all wheeled trees $\Gamma$ in $\mathcal{G}_{n+1, m}$. More precisely, we need to take into account the number of graphs isomorphic to $\Gamma$, for any wheeled tree $\Gamma$ in $\mathcal{G}_{n+1, m}$, since we do not want to count too many wheeled trees.

Since the central vertex of wheeled tree $\Gamma$ is fixed, permutations of the $n$ vertices of the first type of $\Gamma$ induce isomorphic wheeled trees to $\Gamma$. On the other hand, denoting by $\tau_{i}$ the number of wheels of length $i$ of $\Gamma$, it is clear that any permutation of the $\tau_{i}$ wheels produces a wheeled tree isomorphic to $\Gamma$. Further, we have also to keep into account the number of cyclic permutations of the vertices of each wheel: with the same notations as above, for the wheel of length $i$, the number of such permutations, taking into accout that $\Gamma$ contains $\tau_{i}$ wheels of length $i$, is exactly $i^{\tau_{i}}$. Hence, the number of isomorphic wheeled trees with a partition of wheels of the form

$$
\{\underbrace{1, \ldots, 1}_{\tau_{1} \text {-times }}, \underbrace{2, \ldots, 2}_{\tau_{2} \text {-times }}, \ldots, \underbrace{n, \ldots, n}_{\tau_{n} \text {-times }}\}
$$

is given by

$$
\frac{n !}{\prod_{i=1}^{n} \tau_{i} ! \prod_{i=1}^{n} i^{\tau_{i}}} .
$$

We only observe that, if a wheeled tree $\Gamma$ belongs to $\mathcal{G}_{n+1, m}$, the maximal size of a wheel of $\Gamma$ is $n$, by obvious reasons.

Summarizing all these facts, we find the following explicit expression for (5.2):

$$
\begin{aligned}
\mathcal{U}_{Q}(\alpha) & =\sum_{n \geq 0} \frac{1}{\prod_{i=1}^{n} \tau_{i} ! \prod_{i=1}^{n} i^{\tau_{i}}} I_{H K R}\left(X_{1}^{\tau_{1}} \cdots X_{n}^{\tau_{n}}(\alpha)\right)= \\
& =I_{H K R}\left(e^{X_{1}+\frac{X_{2}}{2}+\cdots+\frac{X_{n}}{n}+\cdots}(\alpha)\right),
\end{aligned}
$$

using the previous notations. Further, we may define, for a cohomological vector field $Q$ on $V$, a (formal) contraction operator on $T_{\text {poly }}$ via

$$
\Theta=\sum_{n>0} \frac{1}{n} X_{n}=\sum_{n>0} \frac{1}{n} \mathcal{W}_{\Sigma_{n}} \iota_{\operatorname{tr}\left(\Xi^{n}\right)},
$$

where $\Xi$ is as in (9.3). Thus, using (9.7), we can rewrite finally (9.6) in the considerably simpler form

$$
\mathcal{U}_{Q}(\alpha)=I_{H K R}\left(e^{\Theta}(\alpha)\right)
$$




\subsection{The weight of an even wheel.}

We observe that the differential form (9.7) acts on the polyvector field $\alpha$ by means of contraction. At the end, using results of Cattaneo-Felder-Willwacher [34] and Van den Bergh [32], we can put (5.2) in the form of (9.8), into relationship with the Todd class of $V$.

Theorem 9.3. The following identity holds true, for any choice of a vector field $Q$ on $V$ :

$$
e^{\Theta}=\operatorname{Ber} \sqrt{\frac{e^{\frac{\bar{z}}{2}}-e^{-\frac{\bar{\Xi}}{2}}}{\Xi}}
$$

with $\Xi$ is the supermatrix-valued 1-form introduced in (9.3); Ber denotes the Berezinian of the supervector space $V$, i.e. the superdeterminant of endomorphisms of the superspace $V$.

Remark 9.4. The supermatrix-valued differential form $\frac{e^{\frac{\overline{\frac{E}{2}}}{2}}-e^{-\frac{\bar{z}}{2}}}{\Xi}$ is to be understood as obtained from the power series expansion of the function

$$
B(t)=\frac{e^{\frac{t}{2}}-e^{-\frac{t}{2}}}{t},
$$

putting $\Xi$ instead of $t$. Actually, the previous result may be restated as the following identity for formal power series

$$
\sum_{n>0} \frac{\mathcal{W}_{n}}{n} t^{n}=\frac{1}{2} \log (B(t))
$$

where $\mathcal{W}_{n}$ is the weight of the standard wheel of length $n$.

This ends the proof of Theorem 5.3.

Yet another way of computing wieghts of even wheels.

Actually, Theorem 9.3 can be obtained as a consequence of the standard Duflo Theorem (i.e Theorem 1.2 of the present text). More precisely, Let us consider the case when $V=\Pi \mathfrak{g}$ and $Q$ is the cohomological vector field on $\mathcal{O}_{V}=\wedge\left(\mathfrak{g}^{*}\right)$ is given by the Chevalley-Eilenberg differential, $\mathfrak{g}$ being a finite dimensional Lie algebras.

On one hand, following what we have done in Subsection 5.2, one obtains that $\mathcal{U}_{Q}$ induces an isomorphism of algebras $S(\mathfrak{g})^{\mathfrak{g}} \sim \widetilde{\sim}(\mathfrak{g})^{\mathfrak{g}}$ explicitly given by $I_{P B W} \circ\left(e^{\Theta} \cdot\right)$, with

$$
\Theta=\sum_{n>0} \frac{1}{2 n} \mathcal{W}_{\Sigma_{2 n}} \operatorname{tr}\left(\operatorname{ad}^{2 n}\right)
$$

On the other hand $I_{P B W} \circ\left(\widetilde{j}^{1 / 2} \cdot\right)$ also induces an algebra isomorphism $S(\mathfrak{g})^{\mathfrak{g}} \sim \widetilde{\sim} U(\mathfrak{g})^{\mathfrak{g}}$ (this is precisely the original Duflo Theorem).

We now proceed by induction. Assume that we have proved that $\frac{1}{2 k} \mathcal{W}_{\Sigma_{2 k}}$ coincide with the coefficient $b_{2 k}$ of $t^{2 k}$ in the series $\frac{1}{2} \log (B(t))$ introduced in Remark 9.4 for any $k<n .{ }^{8}$ Observe that since $I_{P B W} \circ\left(e^{\Theta} \cdot\right)$ and $I_{P B W} \circ\left(\widetilde{j}^{1 / 2} \cdot\right)$ are both algebra ismorphisms from $S(\mathfrak{g})^{\mathfrak{g}}$ to $U(\mathfrak{g})^{\mathfrak{g}}$, then the action of the series $\widetilde{j}^{1 / 2} e^{-\Theta}$ defines an algebra automorphism of $S(\mathfrak{g})^{\mathfrak{g}}$.

In particular, the first non-vanshing term, which is, thanks to the induction assumption,

$$
\left(b_{2 n}-\frac{1}{2 n} \mathcal{W}_{\Sigma_{2 n}}\right) \operatorname{tr}\left(\mathrm{ad}^{2 n}\right),
$$

acts as a derivation on the algebra $S(\mathfrak{g})^{\mathfrak{g}}$.

As it is not true that $\operatorname{tr}\left(\operatorname{ad}^{2 n}\right)$ acts as a derivation on $S(\mathfrak{g})^{\mathfrak{g}}$ for any Lie algebra $\mathfrak{g}$ (one can actually check this on $\mathfrak{s l}_{N}(\mathbb{C})$ for $N$ big enough), then one has

$$
b_{2 n}=\frac{1}{2 n} \mathcal{W}_{\Sigma_{2 n}} .
$$

\footnotetext{
${ }^{8}$ Coefficients of odd powers of $t$ in $B(t)$ obviously vanish.
} 


\section{Fedosov RESOLutions}

In this Section we follow [5] in which resolutions of the DG Lie algebras $\left(\Omega^{0, \bullet}\left(M, T_{\text {poly }}^{\prime}\right), \bar{\partial}\right)$ and $\left(\Omega^{0, \bullet}\left(M, D_{\text {poly }}^{\prime}\right), d_{H}+\bar{\partial}\right)$ are constructed when $M$ is a complex manifold. The differentials in these resolutions will be obtained locally through the action of a cohomological vector field so that we will be able to use the Duflo isomorphism for $Q$-spaces to prove Theorem 3.5 .

\subsection{Bundles of formal fiberwise geometric objects.}

In this paragraph we introduce some infinite dimensional bundles that will be of some relevance in the sequel. These bundles (defined in [5]) are straightforward adaptation, in a holomorphic context, of the ones introduced by Dolgushev [11] in his approach to the globalization of Kontsevich's formality theorem. He himself was directly inspired from Fedosov's construction [14] of $*$-products on symplectic manifolds.

All these bundles being made from $T^{\prime}$, they all are holomorphic bundles. Here are their definitions:

- we first consider $\mathcal{O}:=\widehat{S}\left(\left(T^{\prime}\right)^{*}\right)$, the formally completed symmetric algebra bundle of $\left(T^{\prime}\right)^{*}$. Sections of $\mathcal{O}$ are called formal fiberwise functions on $T^{\prime}$, and can be written locally in the following form:

$$
f=\sum_{k \geq 0} f_{i_{1}, \ldots, i_{k}}(z, \bar{z}) y^{i_{1}} \cdots y^{i_{k}}
$$

where $y^{i}=\mathrm{d} z^{i}$ are even coordinates (formal coordinates in the fibers);

- then consider the Lie algebra bundle $\mathcal{T}:=\operatorname{Der}(\mathcal{O})$ of formal fiberwise vector fields on $T^{\prime}$. One has that $\mathcal{T}=\mathcal{O} \otimes T^{\prime}$, and sections can be written locally in the following form:

$$
v=\sum_{k \geq 0} v_{i_{1}, \ldots, i_{k}}^{j}(z, \bar{z}) y^{i_{1}} \cdots y^{i_{k}} \frac{\partial}{\partial y^{j}} ;
$$

- one also has the graded algebra bundle $\mathcal{T}_{\text {poly }}^{\bullet}:=\wedge_{\mathcal{O}}^{\bullet} \mathcal{T}$ of formal fiberwise polyvector fields on $T^{\prime}$. One has $\mathcal{T}_{\text {poly }}^{\bullet}=\mathcal{O} \otimes\left(\wedge^{\bullet}\left(T^{\prime}\right)\right)$, and sections can be written locally in the following form:

$$
v=\sum_{k \geq 0} v_{i_{1}, \ldots, i_{k}}^{j_{1}, \ldots, j_{l}}(z, \bar{z}) y^{i_{1}} \cdots y^{i_{k}} \frac{\partial}{\partial y^{j_{1}}} \wedge \cdots \wedge \frac{\partial}{\partial y^{j_{l}}}
$$

- dualizing w.r.t. $\mathcal{O}$, one obtains the DG algebra bundle $\mathcal{A}^{\bullet}=\mathcal{O} \otimes\left(\wedge^{\bullet}\left(T^{\prime}\right)^{*}\right)$ of formal fiberwise differentiable forms on $T^{\prime}$. Sections have the following local form:

$$
\omega=\sum_{k \geq 0} \omega_{i_{1}, \ldots, i_{k} ; j_{1}, \ldots, j_{l}}(z, \bar{z}) y^{i_{1}} \cdots y^{i_{k}} \mathrm{~d} y^{j_{1}} \wedge \cdots \wedge \mathrm{d} y^{j_{l}} .
$$

One has a fiberwise de Rham differential $\mathrm{d}^{f}:=\mathrm{d} y^{i} \frac{\partial}{\partial y^{i}}$;

- the bundle $\mathcal{D}$ of formal fiberwise differential operators consists of the subalgebra bundle of $\operatorname{End}(\mathcal{O})$ that is generated by $\mathcal{O}$ and $\mathcal{T}$. As a bundle it is $\mathcal{O} \otimes\left(S\left(T^{\prime}\right)\right.$ ) and thus its sections locally looks like as follows:

$$
P=\sum_{k \geq 0} P_{i_{1}, \ldots, i_{k}}^{j_{1}, \ldots, j_{l}}(z, \bar{z}) y^{i_{1}} \cdots y^{i_{k}} \frac{\partial^{j_{1}+\cdots+j_{l}}}{\partial y^{j_{1}} \cdots \partial y^{j_{l}}}
$$

- we finally consider the graded algebra bundle $\mathcal{D}_{\text {poly }}:=\otimes_{\mathcal{O}} \mathcal{D}=\mathcal{O} \otimes\left(\otimes S\left(T^{\prime}\right)\right)$ of formal fiberwise polydifferential operators. One has to be careful about the following: while the product in $\mathcal{D}$ is given by the composition of operators the (graded) product in $\mathcal{D}_{\text {poly }}$ is given by the concatenation of poy-differential operators. We let as an exercise the explicit writting of the local expression of sections of $\mathcal{D}_{\text {poly }}$.

Observe that the Lie algebra bundle $\mathcal{T}$ acts on all these (possibly graded) bundles: 
- it acts on $\mathcal{O}$ by derivations (this is the definition of $\mathcal{T}$ ),

- it acts on itself by the adjoint action,

- as usual the action on $\mathcal{O}$ and $\mathcal{T}$ can be extended by derivations to an action on $\mathcal{T}_{\text {poly }}^{\bullet}$,

- $\mathcal{T}$ also acts on $\mathcal{A}^{\bullet}$ by the (fiberwise) Lie derivative,

- it also acts on $\mathcal{D}$ by taking the commutator,

- as usual the action on $\mathcal{O}$ and $\mathcal{D}$ can be extended by derivations to an action on $\mathcal{D}_{\text {poly }}^{\bullet}$.

Remark 10.1. Observe that, given a connection $\nabla=\nabla^{\prime}+\bar{\partial}$ compatible with the complex structure on $T^{\prime}$, then one can identify $D^{\prime}$ with $S\left(T^{\prime}\right)$. Moreover, this identification commutes with the action of $\bar{\partial}$ on both sides (i.e. it is a morphism of holomorphic bundles). Nevertheless, such an identification does NOT respect the product on both sides (since it is commutative only on one side).

\subsection{Resolutions of algebras.}

In this paragraph $\mathcal{B}$ (resp. $B$ ) will denote any of the $\mathcal{O}$-modules $\mathcal{O}, \mathcal{T}, \mathcal{T}_{\text {poly }}, \mathcal{A}, \mathcal{D}$ or $\mathcal{D}_{\text {poly }}$ (resp. the bundles $\mathbb{C},{ }^{9} T^{\prime}, T_{\text {poly }}^{\prime}, \wedge\left(T^{\prime}\right)^{*}, \mathcal{D}^{\prime}$ or $D_{\text {poly }}^{\prime}$ ).

Let us consider the one-form valued fiberwise vector field $\theta:=\mathrm{d} z^{i} \frac{\partial}{\partial y^{i}}$, which is nothing but the identity tensor id $\in\left(T^{\prime}\right)^{*} \otimes T^{\prime}$, and write $\delta:=\theta$. for the degree one derivation of $\Omega^{\bullet}, q(M, \mathcal{B}), q \geq 0$, given by the action of $\theta$ on it. It is an obvious fact that $\delta \circ \delta=0$, i.e. $\delta$ is a differential.

Proposition 10.2. 1. $H^{p}\left(\Omega^{\bullet}, q(M, \mathcal{B}), \delta\right)=\{0\}$ for $p>0$.

2. $H^{0}\left(\Omega^{\bullet}, q(M, \mathcal{B}), \delta\right)=\Omega^{0, q}(M, \mathcal{B}) \cap(\operatorname{ker} \delta)$.

3. In case $\mathcal{B}$ is an algebra bundle the previous equality is an equality of algebras.

Proof. This is the Poincaré lemma (see example 1.7)! Namely, we define a degree -1 graded $\Omega^{0, q}(M)$-linear endomorphism $\kappa$ of $\Omega^{\bullet, q}(M, \mathcal{B})$ as follows: $\kappa(1)=0$ and

$$
\kappa_{\mid \operatorname{ker}(p)}(f(\underline{y}, \mathrm{~d} \underline{z}))=y^{i} \iota \frac{\partial}{\partial z^{i}}\left(\int_{t=0}^{1} f(t \underline{y}, t \mathrm{~d} \underline{z}) \frac{d t}{t}\right),
$$

where $p: \Omega^{\bullet}, q(M, \mathcal{B}) \rightarrow \Omega^{0, q}(M, \mathcal{B}) \cap(\operatorname{ker} \delta)$ is the projection on $(0, q)$-forms that are constant in the fibers; i.e. $p(f(\underline{y}, \mathrm{~d} \underline{z})=f(0,0)$. As for the proof of the Poincaré lemma $\kappa$ is a homotopy operator: it satisfies

$$
\delta \circ \kappa+\kappa \circ \delta=\mathrm{id}-i \circ p,
$$

where $i: \Omega^{0, q}(M, \mathcal{B}) \cap(\operatorname{ker} \delta) \rightarrow \Omega^{\bullet}, q(M, \mathcal{B})$ is the natural inclusion of $\mathcal{B}$-valued $(0, q)$-forms that are constant in the fibers into $\Omega^{\bullet}, q(M, \mathcal{B})$.

Finally, in the case $\mathcal{B}$ is an algebra bundle $i$ and $p$ are algebra morphisms.

Observe that one also has $\kappa \circ \kappa=0$. This fact will be very useful below. Observe also that $\delta$ commutes with $\bar{\partial}$, which means that we have injective quasi-isomorphisms $i$ : $\left(\Omega^{0, \bullet}(M, \mathcal{B}) \cap \operatorname{ker} \delta, \bar{\partial}\right) \hookrightarrow\left(\Omega^{\bullet}(M, \mathcal{B}), \bar{\partial}-\delta\right)$.

One has obvious isomorphisms $\mathcal{B} \cap(\operatorname{ker} \delta) \cong B$ of holomorphic bundles. ${ }^{10}$ Nevertheless if $\mathcal{B}$ is $\mathcal{T}$, resp. $\mathcal{D}$, and $B$ is $T^{\prime}$, resp. $D^{\prime}$, then it does not respect the Lie bracket, resp. the product.

We will remedy to this problem in the remainder of this section. More generally we will perturb $\bar{\partial}-\delta$ and $i$ to a new differential $D$ on $\Omega^{\bullet}(M, \mathcal{B})$ and a new injective quasiisomorphism $\lambda:\left(\Omega^{0, \bullet}(M, B), \bar{\partial}\right) \hookrightarrow\left(\Omega^{\bullet}(M, \mathcal{B}), D\right)$ that intertwines the $T^{\prime}$ - and $\mathcal{T}$-actions and respects all algebraic structures.

\footnotetext{
${ }^{9}$ Here $\mathbb{C}$ is considered as a bundle, the trivial line bundle on $M$, whose sections are functions on $M$.

${ }^{10}$ In the case when $\mathcal{B}$ is $\mathcal{D}$, resp. $\mathcal{D}_{\text {poly }}$, and $B$ is $D^{\prime}$, resp. $D_{\text {poly }}^{\prime}$, one needs to use the identification of Remark 10.1.
} 


\subsection{Fedosov differential.}

We keep the notations of the previous paragraph and assume that $\nabla=\nabla^{\prime}+\bar{\partial}$ is a connection compatible with the complex structure on $T^{\prime}$.

Thanks to the pairing between $T^{\prime}$ and $\left(T^{\prime}\right)^{*}, \nabla$ defines a connection compatible with the complex structure on $\left(T^{\prime}\right)^{*}$ : for any $v \in \Gamma\left(M, T^{\prime}\right)$ and $\xi \in \Gamma\left(M,\left(T^{\prime}\right)^{*}\right)$ one has

$$
\langle\nabla(\xi), v\rangle=\mathrm{d}\langle\xi, v\rangle-\langle\xi, \nabla(u)\rangle .
$$

We then extend it by derivations to a connection compatible with the complex structure on $\mathcal{O}=\widehat{S}\left(\left(T^{\prime}\right)^{*}\right)$; it it thus locally given by the following formula:

$$
\nabla=\partial+\bar{\partial}-\mathrm{d} z^{i} \Gamma_{i j}^{k}(z, \bar{z}) y^{j} \frac{\partial}{\partial y^{k}}
$$

Formula (10.2) finally extends to a connection compatible with the complex structure on any of the bundles $\mathcal{B}$, thanks to the $\mathcal{T}$-module structure on them. Therefore $\nabla$ defines a degree one derivation of the graded algebra $\Omega^{\bullet}(M, \mathcal{B})$.

Lemma 10.3. One can always assume that $\nabla$ has zero torsion. In this case $\nabla \delta+\delta \nabla=0$.

Before proving the lemma we remind to the reader that the torsion of a connection compatible with the complex structure on $T^{\prime}$ is the tensor $T \in \Omega^{2,0}\left(M, T^{\prime}\right)$ defined by $T(u, v):=\nabla_{u}^{\prime} v-\nabla_{v}^{\prime} u-[u, v]$. Locally one has $T_{i j}^{k}=\Gamma_{i j}^{k}-\Gamma_{j i}^{k}$.

Proof. Locally the zero torsion condition can be written as follows: $\Gamma_{i j}^{k}-\Gamma_{j i}^{k}=0$. Therefore one sees that a connection compatible with the complex structure on $T^{\prime}$ having zero torsion always exists. Namely, given a covering $\left(U_{\alpha}\right)_{\alpha}$ of $M$ by trivializing opens one defines $\nabla_{\alpha}$ by taking $\left(\Gamma_{\alpha}\right)_{i j}^{k}=0$. Let then $\left(f_{\alpha}\right)_{\alpha}$ be a partition of unity and defines $\nabla:=\sum_{\alpha} f_{\alpha} \nabla_{\alpha}$.

Now we assume $\nabla$ has zero torsion and compute: since $d=\partial+\bar{\partial}$ obviously commutes with $\delta$ one has

$$
\nabla \circ \delta+\delta \circ \nabla=\left[\mathrm{d} z^{i} \Gamma_{i j}^{k} y^{j} \frac{\partial}{\partial y^{k}}, \mathrm{~d} z^{l} \frac{\partial}{\partial y^{l}}\right] \cdot=-\mathrm{d} z^{i} \wedge \mathrm{d} z^{j} \Gamma_{i j}^{k} \frac{\partial}{\partial y^{k}} \cdot=0
$$

This ends the proof of the lemma.

From now we assume that $\nabla$ has zero torsion.

Let $R=R^{2,0}+R^{1,1} \in \Omega^{2}\left(M, \operatorname{End}\left(\left(T^{\prime}\right)^{*}\right)\right.$ be the curvature tensor of $\nabla$. Then $\nabla \circ \nabla$ acts on $\Omega^{\bullet}(M, \mathcal{B})$ as $-R \cdot=-R_{k}^{l} y^{k} \frac{\partial}{\partial y^{l}} \cdot ;$ in other words

$$
-\left(\frac{1}{2} \mathrm{~d} z^{i} \wedge \mathrm{d} z^{j}\left(R^{2,0}\right)_{i j_{k}}{ }^{l}+\mathrm{d} \bar{z}^{i} \wedge \mathrm{d} z^{j}\left(R^{1,1}\right)_{i j k}{ }^{l}\right) y^{k} \frac{\partial}{\partial y^{l}} .
$$

Theorem 10.4. There exists an element $A \in \Omega^{1}\left(M, \mathcal{T}_{\geq 2}\right)$ as in such that $\kappa A=0$ and the corresponding derivation $D:=\nabla-\delta+A$. has square zero: $D \circ D=0$.

Before proving the theorem let us observe that there is a filtration on the bundle $\mathcal{O}$ that is given by the polynomial (i.e. symmetric) degree in the fibers (i.e. in $y$ 's). It induces a filtration on $\mathcal{B}$ (including $\mathcal{T}$ ). This is the filtration we consider in the statement and proof of the theorem.

Proof. Since $\kappa$ raises the degree in the filtration there is a unique solution $A \in \Omega^{1}(M, \mathcal{B})$ to the following equation:

$$
A=\kappa\left(-R+\nabla A+\frac{1}{2}[A, A]\right)
$$

First observe that $\kappa \circ \kappa=0$ implies that $\kappa(A)=0$. Now let us show that $A$ satisfies equation

$$
-R+\nabla A-\delta A+\frac{1}{2}[A, A]=0,
$$


which obviously implies that $D \circ D=0$. Using (10.1) together with $\kappa(A)=0=p(A)$ one finds that

$$
\kappa \delta A=\kappa\left(-R+\nabla A+\frac{1}{2}[A, A]\right)
$$

Define $C:=-R+\nabla A-\delta A+\frac{1}{2}[A, A]$. One can rewrite Bianchi identities for $\nabla$ in the following way: $\delta R=0=\nabla R$. Thanks to these equalities and (10.1) on has

$$
\nabla C-\delta C=(\nabla-\delta)\left(\frac{1}{2}[A, A]\right)-[R, A]=[\nabla A-\delta A-R, A]=[C, A],
$$

where the last equality follows from the (super-)Jacobi identity. Finally, due to (10.5) one has $\kappa C=0$ and thus $C=\kappa(\nabla C+[A, C])$. Since the operator $\kappa$ raises the degree in the filtration this latter equation has a unique solution, that is zero. Thus $A$ satisfies (10.4) and the theorem is proved.

$D$ is refered to as the Fedosov differential.

\subsection{Fedosov resolutions.}

We keep the notations of the previous paragraphs.

Theorem 10.5. There exists quasi-isomorphisms $\ell:\left(\Omega^{0, *}(M, B), \bar{\partial}\right) \rightarrow\left(\Omega^{*}(M, \mathcal{B}), D\right)$ with the following properties:

(1) $\ell$ is $\Omega^{0, *}(M)$-linear;

(2) if $\mathcal{B} \neq \mathcal{T}$ then $\ell$ is a graded associative algebra morphism;

(3) if $\mathcal{B}=\mathcal{D}_{\text {poly }}$ then $\ell$ commutes with Hochschild differentials and thus becomes a quasiisomorphism $\left(\Omega^{0, *}(M, B), \bar{\partial}+d_{H}\right) \rightarrow\left(\Omega^{*}(M, \mathcal{B}), D+d_{H}\right)$;

(4) $\ell$ is compatible with the contraction of polyvector fields by forms.

Proof. We first prove that $H^{\bullet}\left(\Omega^{*}(M, \mathcal{B}), D\right)=H^{\bullet}\left(\Omega^{0, *}(M, \mathcal{B}) \cap(\operatorname{ker} \delta), \bar{\partial}\right)$.

Observe that $D=D^{\prime}+D^{\prime \prime}$, with $D^{\prime}: \Omega^{*, *}(M, \mathcal{B}) \rightarrow \Omega^{*+1, *}(M, \mathcal{B})$ and $D^{\prime \prime}: \Omega^{*, *}(M, \mathcal{B}) \rightarrow$ $\Omega^{*, *+1}(M, \mathcal{B})$, and let us compute the cohomology with respect to $D^{\prime}$. We consider the spectral sequence associated to the filtration given by the degree in the fibers, for which $D^{\prime}$ decreases the degree by one. We have $d_{-1}=-\delta$. Therefore thanks to Proposition 10.2

$$
E_{0}^{\bullet, \bullet}=E_{0}^{0,0}=\Omega^{0, *}(M, \mathcal{B}) \cap(\operatorname{ker} \delta),
$$

and thus $H^{\bullet}\left(\Omega^{*}(M, \mathcal{B}), D^{\prime}\right)=H^{0}\left(\Omega^{*}(M, \mathcal{B}), D^{\prime}\right)=\Omega^{0, *}(M, \mathcal{B}) \cap\left(\operatorname{ker} D^{\prime}\right)$.

Now since the $D^{\prime}$-cohomology is concentrated in degree zero then the $D$-cohomology, which is the cohomology of the double complex $\left(\Omega^{\bullet \bullet \bullet}(M, \mathcal{B}), D^{\prime}, D^{\prime \prime}\right)$, is

$$
H^{\bullet}\left(\Omega^{*}(M, \mathcal{B}), D\right)=H^{\bullet}\left(H^{0}\left(\Omega^{*}(M, \mathcal{B}), D^{\prime}\right), D^{\prime \prime}\right)=H^{\bullet}\left(\Omega^{0, *}(M, \mathcal{B}) \cap\left(\operatorname{ker} D^{\prime}\right), D^{\prime \prime}\right) .
$$

We then construct an isomorphism of complexes

$$
\lambda:\left(\Omega^{0, \bullet}(M, \mathcal{B}) \cap(\operatorname{ker} \delta), \bar{\partial}\right) \longrightarrow\left(\Omega^{0, \bullet}(M, \mathcal{B}) \cap\left(\operatorname{ker} D^{\prime}\right), D^{\prime \prime}\right) .
$$

For any $u \in \Omega^{0, \bullet}(M, \mathcal{B})$ such that $\delta(u)=0$ we define

$$
\lambda(u):=u+\kappa\left(\left(D^{\prime}+\delta\right)(\lambda(u))\right) .
$$

This is well-defined (by iteration) since $\kappa$ raises the filtration degree and $D^{\prime}+\delta$ respects it. Thanks to $\kappa(u)=0, p(\lambda(u))=u, \kappa \circ \kappa=0$ and equation (10.1), one has

$$
\kappa\left(D^{\prime}(\lambda(u))\right)=\kappa\left(\left(D^{\prime}+\delta\right)(\lambda(u))\right)-\kappa(\delta(\lambda(u)))=(\lambda(u)-u)-(\lambda(u)-u)=0 .
$$

Setting $Y:=D^{\prime}(\lambda(u))$ one obtains $\kappa(Y)=0$ and $\delta(Y)=\left(D^{\prime}+\delta\right)(Y)$. Therefore using (10.1) again we see that

$$
Y=\kappa\left(\left(D^{\prime}+\delta\right)(Y)\right)
$$


which admits 0 as a unique solution (since $\kappa$ raises the filtration degree). Consequently, $D^{\prime}(\lambda(u))=0 . \lambda$ is an isomorphism of graded vector spaces with $\lambda^{-1}=p$. Moreover $p$ (and so $\lambda$ ) is obviously a morphism of complexes since $D^{\prime \prime}$ is given by $\bar{\partial}$ plus something that raises the filtration degree.

Finally, composing $\lambda$ with the isomorphism $\mathcal{B} \cap(\operatorname{ker} \delta) \cong B$, we obtain the desired quasiisomorphism $\ell$, which is obviously $\Omega^{0, *}(M)$-linear.

Since $\mathcal{B} \cap(\operatorname{ker} \delta) \cong B$ is an algebra bundle isomorphism when $\mathcal{B}$ is either $\mathcal{O}, \mathcal{T}_{\text {poly }}, \mathcal{A}$ or $\mathcal{D}_{\text {poly }}$ then the second property is satisfied in these cases. Moreover, the fourth property is also obviously satisfied.

We now consider the situation when $\mathcal{B}=\mathcal{D}$.

Lemma 10.6. Let $f, g \in C^{\infty}(M)$ and $u, v \in \Gamma\left(M, T^{\prime}\right)$. Then $\ell(f g)=\ell(f) \ell(g), \ell(f v)=$ $\ell(f) \ell(v), \ell(v \cdot f)=\ell(v) \cdot \ell(f)$ and $\ell([u, v])=[\ell(u), \ell(v)]$.

Proof of the lemma. There are only two non trivial equalities to check: $\ell(v \cdot f)=\ell(v) \cdot \ell(f)$ and $\ell([u, v])=[\ell(u), \ell(v)]$. First observe that

$\ell(f)=f+y^{i} \frac{\partial f}{\partial z^{i}}+O\left(|y|^{2}\right) \quad$ and $\quad \ell(u)=\ell\left(u^{i} \frac{\partial}{\partial z^{i}}\right)=u^{i} \frac{\partial}{\partial y^{i}}+y^{i}\left(\frac{\partial u^{k}}{\partial z^{i}}+u^{j} \Gamma_{i j}^{k}\right) \frac{\partial}{\partial y^{k}}+O\left(|y|^{2}\right)$.

Then compute:

$$
\ell(u) \cdot \ell(f)=u^{i} \frac{\partial f}{\partial z^{i}}+O(|y|)=u \cdot f+O(|y|)=\ell(u \cdot f)
$$

and

$$
\begin{aligned}
{[\ell(u), \ell(v)] } & =u^{i}\left(\frac{\partial v^{k}}{\partial z^{i}}+v^{j} \Gamma_{i j}^{k}\right) \frac{\partial}{\partial y^{k}}-v^{i}\left(\frac{\partial u^{k}}{\partial z^{i}}+u^{j} \Gamma_{i j}^{k}\right) \frac{\partial}{\partial y^{k}}+O(|y|) \\
& =\left(u^{i} \frac{\partial v^{k}}{\partial z^{i}}-v^{i} \frac{\partial u^{k}}{\partial z^{i}}\right) \frac{\partial}{\partial y^{k}}+O(|y|) \\
& =[u, v]^{k} \frac{\partial}{\partial y^{k}}+O(|y|)=\ell([u, v])
\end{aligned}
$$

The lemma is proved.

The algebra of $\bar{\partial}$-differential operators is generated by $C^{\infty}(M)$ and $\Gamma\left(M, T^{\prime}\right)$, and the defining relations are $f * g=f g, f * u=f u, u * f-f * u=u \cdot f$ and $u * v-v * u=[u, v]$. Therefore the lemma proves that $\ell$ is an algebra morphism.

Moreover, it implies that for any $\bar{\partial}$-diffferential operator $P$ and any function $f$ one has

$$
\ell(P(f))=\ell(P)(\ell(f)) .
$$

This last identity can be used to prove that $\ell$ commutes with Hochschild differentials when $\mathcal{B}=\mathcal{D}_{\text {poly }}$ (this is the third property). This ends the proof of the theorem.

\subsection{Proof of Theorem 3.5.}

Observe that $D$ is locally given on any holomorphic coordinate chart $U$ by the following formula:

$$
D=\partial+\bar{\partial}+Q_{U}
$$

where $Q_{U} \in \Omega^{1}(U, \mathcal{T})$. The square zero property of $D$ tells us that $Q_{U}$ satisfies the MaurerCartan equation

$$
(\partial+\bar{\partial})\left(Q_{U}\right)+\frac{1}{2}\left[Q_{U}, Q_{U}\right]=0 .
$$

One can therefore apply Theorem 5.3 and thus obtain a quasi-isomorphism

$$
\mathcal{U}_{Q_{U}}:\left(\Omega\left(U, \mathcal{T}_{\text {poly }}\right), \partial+\bar{\partial}\right) \longrightarrow\left(\Omega\left(U, \mathcal{D}_{\text {poly }}\right), \partial+\bar{\partial}+d_{H}\right)
$$


that induces an algebra isomorphism in cohomology. Let us remind to the reader that $\mathcal{U}_{Q_{U}}$ is given by the fiberwise HKR map $\mathcal{T}_{\text {poly }} \rightarrow \mathcal{D}_{\text {poly }}$ composed with

$$
\left.\operatorname{det}\left(\frac{\Xi_{U}}{e^{\Xi_{U} / 2}-e^{-\Xi_{U} / 2}}\right) \in \bigoplus_{k} \Omega^{k}\left(U, \mathcal{A}^{k}\right) \quad \text { (remember that }\left(\Xi_{U}\right)_{i}^{j}:=\mathrm{d}\left(\frac{\partial Q_{U}^{j}}{\partial y^{i}}\right)\right)
$$

acting on $\Omega\left(U, \mathcal{T}_{\text {poly }}\right)$. On an intersection $U \cap V$ the difference $Q_{U}-Q_{V}$ is a linear vector field, and thus $\Xi_{U}-\Xi_{V}=0$. In particular $\mathcal{U}_{Q_{U}}$ and $\mathcal{U}_{Q_{V}}$ coincide on $U \cap V$; one therefore has a globally well-defined quasi-isomorphism

$$
\mathcal{U}_{Q}:\left(\Omega\left(M, \mathcal{T}_{\text {poly }}\right), \partial+\bar{\partial}\right) \longrightarrow\left(\Omega\left(M, \mathcal{D}_{\text {poly }}\right), \partial+\bar{\partial}+d_{H}\right) .
$$

Proposition 10.7. $\mathcal{U}_{Q}$ induces an algebra isomorphism in cohomology.

Proof. On each holomorphic coordinate chart $U$ there is a homotopy $\mathcal{H}_{Q_{U}}$. On an intersection $U \cap V$ one has

$$
\begin{aligned}
\mathcal{H}_{Q_{U}}(\alpha, \beta) & =\sum_{n \geq 0} \frac{1}{n !} \sum_{\Gamma \in G_{n+2, m}} \widetilde{\mathcal{W}}_{\Gamma} \mathcal{B}_{\Gamma}\left(\alpha, \beta, Q_{U}, \ldots, Q_{U}\right) \\
& =\sum_{n \geq 0} \frac{1}{n !} \sum_{\Gamma \in G_{n+2, m}} \widetilde{\mathcal{W}}_{\Gamma} \mathcal{B}_{\Gamma}\left(\alpha, \beta, Q_{V}, \ldots, Q_{V}\right)=\mathcal{H}_{Q_{V}}(\alpha, \beta),
\end{aligned}
$$

where the second equality follow from the fact that $\widetilde{\mathcal{W}}_{\Gamma} \mathcal{B}_{\Gamma}$ vanishes if at least one argument is a linear vector field (thansk to Lemma 6.9 ). We therefore have a globally well-defined homotopy $\mathcal{H}_{Q}$.

Remember that thanks to Theorem $10.5 \ell$ defines a quasi-isomorphism

$$
\left(\Omega^{0, *}\left(M, \wedge\left(T^{\prime}\right)^{*} \otimes \operatorname{End}\left(T^{\prime}\right)\right), \bar{\partial}\right) \longrightarrow(\Omega(M, \mathcal{A} \otimes \operatorname{End}(\mathcal{T})), D),
$$

and one can check that it commutes with det. Therefore, to end the proof of Theorem 3.5, it remains to prove that the class of $\Xi$ is the Atiyah class:

Proposition 10.8. $[\Xi]=\mathrm{at}_{T^{\prime}}$.

Proof. A direct computation using the recursion relation (10.3) shows that

$$
A=\left(\frac{1}{2} \mathrm{~d} z^{i}\left(R^{2,0}\right)_{i j_{k}}^{l}+\mathrm{d} \bar{z}^{i}\left(R^{1,1}\right)_{i j_{k}}^{l}\right) y^{j} y^{k} \frac{\partial}{\partial y^{l}}+O\left(|y|^{3}\right) .
$$

Therefore applying the morphism $p$ (that sends $\mathrm{d} z^{i}$ and $y^{i}$ onto zero) to the matrix element

$$
\Xi_{k}^{l}:=\mathrm{d}\left(\frac{\partial Q^{l}}{\partial y^{k}}\right)=\mathrm{d}\left(\frac{\partial A^{l}}{\partial y^{k}}\right)
$$

one gets

$$
p\left(\Xi_{k}^{l}\right)=\mathrm{d} y^{j} \mathrm{~d} \bar{z}^{i}\left(\left(R^{1,1}\right)_{i j_{k}}^{l}+\left(R^{1,1}\right)_{i k_{j}}^{l}\right) .
$$

The proposition is proved. 


\section{Appendix A. Deformation-theoretical intepretation of the Hochschild COHOMOLOGY OF A COMPLEX MANIFOLD}

In this appendix, we discuss, from the point of view of Čech cohomology, an interpretation of the second Hochschild cohomology group of $X$ in the framework of deformation theory. This is in a certain sense analogous to the deformation-theoretical interpretation of the Hochschild cohomology of an associative algebra $A$ given by Gerstenhaber and sketched in paragraph 2.1.

For a complex manifold $X$, we denote by $\mathcal{D}_{\text {poly }}^{\prime}$ the holomorphic differential graded algebra bundle of polydifferential operators on $X$ of type $(0,1)$, i.e. the local holomorphic sections of $\mathcal{D}_{\text {poly }}^{\prime}$ are holomorphic differential operators on $X$; the differential of $\mathcal{D}_{\text {poly }}^{\prime}$ is the Hochschild differential, denoted by $d_{H}$.

Definition A.1. The Hochschild cohomology of the complex manifold $X$ is the total cohomology of the double complex

$$
\left(\Omega^{(0, \bullet)}\left(X, \mathcal{D}_{\text {poly }}^{\prime}\right), \bar{\partial} \pm d_{H}\right)
$$

\section{A.1. Čech cohomology: a (very) brief introduction.}

We consider a general sheaf $\mathcal{E}$ of abelian groups over a topological space $X$. Additionally, we consider an open covering $\mathfrak{U}$ of $X$.

Definition A.2. The $\check{C}$ ech complex of $\mathcal{E}$ w.r.t. $\mathfrak{U}$, denoted by $\check{C} \bullet(\mathfrak{U}, \mathcal{E})$, is defined as

$$
\check{C}^{p}(\mathfrak{U}, \mathcal{E})=\prod_{i_{0}, \ldots, i_{p}} \mathcal{E}\left(U_{i_{0}} \cap \cdots \cap U_{i_{p}}\right),
$$

where the product is over all $p+1$-tuples of indices for elements of $\mathfrak{U}$, such that all indices are distinct. The $\check{C}$ ech differential $\check{\delta}$ is given explicitly by the formula

$$
(\check{\delta} \alpha)_{i_{0}, \ldots, i_{p+1}}:=\sum_{j=0}^{p+1}(-1)^{j} \alpha_{i_{0}, \ldots, \widehat{i_{j}}, \ldots, i_{p+1}},
$$

where, to keep notations simple, we have omitted to write down the restriction maps. The corresponding cohomology groups $\check{H}^{\bullet}(\mathfrak{U}, \mathcal{E})$ form the $\check{C}$ ech cohomology of $\mathcal{E}$ w.r.t. the open covering $\mathfrak{U}$.

Thus, a Čech cochain $\alpha$ of degree $p$ consists of a family of local sections of $\mathcal{E}$ over all non-trivial intersections of distinct open sets in $\mathfrak{U}$. It is possible to show that, in fact, the Čech complex, as introduced in Definition A.2, is quasi-isomorphic to the Čech complex with the same differential, but whose cochains satisfy an antisymmetry relation w.r.t. the indices, i.e. for which we have

$$
\alpha_{\sigma\left(i_{0}\right), \ldots, \sigma\left(i_{p}\right)}=(-1)^{\sigma} \alpha_{i_{0}, \ldots, i_{p}}, \quad \sigma \in \mathfrak{S}_{p+1} .
$$

Further, we see that Čech cohomology depends on the choice of an open covering of $X$. In order to define the Cech cohomology $\check{H}(X, \mathcal{E})$ of $X$ with values in $\mathcal{E}$, we need the notion of refinement of coverings: without going into the details, an open covering $\mathfrak{V}$ is finer than $\mathfrak{U}$, if for any open subset $V_{j}$ in $\mathfrak{V}$, there is an open subset $U_{f(j)}$ in $\mathfrak{U}$, which contains $V_{j}$. The notion of refinement of coverings yields in turn a structure of direct system on Cech cohomology w.r.t. open coverings, thus allowing to define the Čech cohomology $\check{H} \bullet(X, \mathcal{E})$ of $X$ with values in $\mathcal{E}$ as the direct limit

$$
\check{H}^{\bullet}(X, \mathcal{E})=\lim _{\overrightarrow{\mathcal{U}}} \check{H}^{\bullet}(\mathfrak{U}, \mathcal{E}),
$$

w.r.t. the direct limit structure sketched above.

For completeness, we cite the (adapted version of the) famous Leray's Theorem on sheaf cohomology. 
Theorem A.3 (Leray). If $\mathfrak{U}$ is an acyclic open covering of $X$, i.e.

$$
\check{H}^{p}\left(U_{i_{0}} \cap \cdots \cap U_{i_{q}}, \mathcal{E}\right)=0, \quad p \geq 1,
$$

and for any non-trivial multiple intersection $U_{i_{0}} \cap \cdots \cap U_{i_{q}}$ of open sets in $\mathfrak{U}$, then

$$
\check{H}^{\bullet}(\mathfrak{U}, \mathcal{E}) \cong \check{H}^{\bullet}(X, \mathcal{E}) .
$$

Remark A.4. We may also speak, a bit improperly, of $\check{H} \bullet(X, \mathcal{E})$ as of the sheaf cohomology of $X$ with values in $\mathcal{E}$. More precisely, the sheaf cohomology of $X$ with values in a sheaf $\mathcal{E}$ of abelian groups is defined by means of the right derived functor of the global section functor, which, to a sheaf $\mathcal{E}$ of abelian groups, associates the set of its global sections. Then, the more general version of Theorem A.3 gives an identification between the sheaf cohomology of $X$ with values in a sheaf $\mathcal{E}$ of abelian groups and the Čech cohomology of $X$ w.r.t. an acyclic open covering of $X$ with values in $\mathcal{E}$.

\section{A.2. The link between Čech and Dolbeault cohomology: Dolbeault Theorem.}

We assume now $X$ to be a complex manifold, and we assume $E \rightarrow X$ to be a holomorphic vector bundle over $X$. We want to build a relationship between Dolbeault cohomology of $X$ with values in $E$ and sheaf cohomology of $X$ with values in the sheaf $\mathcal{E}$ of local holomorphic sections of $E$. For a complex manifold $X$, we denote by $\mathcal{O}_{X}$ the structure sheaf of $X$, i.e. the sheaf, whose local sections are local holomorphic functions on $X$ : the corresponding holomorphic bundle is the trivial line bundle over $X$.

First of all, we need a complex version of Poincaré's Lemma, which we state without proof, referring e.g. to the 0 -th chapter of [17].

Lemma A.5. If $U$ is an open polydisk in $\mathbb{C}^{n}$, then

$$
H \frac{\bullet}{\partial}(U)=H \frac{0}{\partial}(U)=\mathcal{O}(U) .
$$

In other words, the Dolbeault complex of a polydisk in $\mathbb{C}^{n}$ is acyclic.

Using Lemma A.5, we get Dolbeault's Theorem, whose proof we only sketch, referring, once again, to the 0 -th chapter of [17].

Theorem A.6 (Dolbeault). Using the same notations as at the beginning of the Subsection, we have the isomorphism

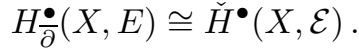

Proof. We consider a sufficiently nice open covering $\mathfrak{U}$ of $X$, i.e. an open covering of $X$ by holomorphic charts of $X$ (e.g. by polydisk charts) and simultaneously by local holomorphic trivializations of $E$. Since $X$ is paracompact, the open covering $\mathfrak{U}$ is also locally finite.

The Cech-Dolbeault double complex of $X$ w.r.t. $\mathfrak{U}$ with values in $E$ is defined as

$$
\left(\check{C}^{\bullet}\left(\mathfrak{U}, \Omega_{E}^{(0, \bullet)}\right), \check{\delta} \pm \bar{\partial}\right)
$$

where $\Omega_{E}^{(0, \bullet)}$ denotes the sheaf of smooth forms of type $(0, \bullet)$ on $X$ with values in $E$. To the Cech-Dolbeault double complex we can associate two natural spectral sequences, according to the two gradations. The "first" degree is the Cech degree, while the "second" degree is the one coming from the Dolbeault complex.

i) The first spectral sequence is associated to the filtration w.r.t. the second degree. The 0 -the term of the spectral sequence is therefore the Čech complex of $\Omega_{E}^{(0, \bullet)}$ w.r.t. $\mathfrak{U}$, hence the first term $E_{1}$ is the Čech cohomology of $\Omega_{E}^{(0, \bullet)}$ w.r.t. $\mathfrak{U}$, which is localized in degree 0 , since $\Omega_{E}^{(0, \bullet)}$ is a sheaf of smooth forms and $X$ admits a smooth partition of unity:

$$
E_{1}=\check{H}^{\bullet}\left(\mathfrak{U}, \Omega_{E}^{(0, \bullet)}\right)=\check{H}^{0}\left(\mathfrak{U}, \Omega_{E}^{(0, \bullet)}\right)=\Omega^{(0, \bullet)}(X, E)
$$


The corresponding differential $d_{1}$ coincides therefore with the Dolbeault differential $\bar{\partial}$, and the spectral sequence abuts at the second term $E_{2}$, which equals then

$$
E_{2}=H \frac{\bullet}{\partial}(X, E) .
$$

ii) The second spectral sequence is associated to the filtration w.r.t. the first degree. The corresponding 0 -th term is the Dolbeault complex on multiple non-trivial intersections of open subsets of $\mathfrak{U}$, hence the first term is, by means of Lemma A.5,

$$
E_{1}=\check{C}^{\bullet}(\mathfrak{U}, H \check{\partial})=\check{C}^{\bullet}(\mathfrak{U}, \mathcal{E}) \text {. }
$$

Lemma A.5 can be applied to this situation, since the open sets of $\mathfrak{U}$ locally trivialize the holomorphic bundle $E$. The corresponding differential $d_{1}$ corresponds to the Čech differential $\tilde{\delta}$. Hence, the spectral sequence abuts also at the second term $E_{2}$, which then equals the Čech cohomology of $X$ w.r.t. $\mathfrak{U}$ with values in $\mathcal{E}$. If, additionally, the open covering $\mathfrak{U}$ is acyclic in the sense of Theorem A.3, then the latter cohomology coincides with the sheaf cohomology of $\mathcal{E}$.

The claim follows then by general arguments on spectral sequences.

We observe that we can consider the more general situation of a differential graded holomorphic vector bundle $\left(E^{\bullet}, d_{E}\right)$ over $X$ : Theorem A.6 can be further generalized as

$$
H \bullet(X, E) \cong \mathbb{H}^{\bullet}(X, \mathcal{E}),
$$

where $H_{\bar{\partial}}^{\bullet}(X, E)$ denotes the total cohomology of the Dolbeault double complex

$$
\left(\Omega^{(0, \bullet)}\left(X, E^{\bullet}\right), \bar{\partial} \pm d_{E}\right)
$$

and, denoting by $\mathcal{E}$ the complex (w.r.t. the differential $d_{E}$ ) of sheaves of local holomorphic sections of $E, \mathbb{H}^{\bullet}(X, \mathcal{E})$ denotes the hypercohomology of $X$ with values in $\mathcal{E}$. The latter cohomology is defined, in this framework, as the total cohomology of the Čech complex associated to $\mathcal{E}$. Considering the generalization (A.1) of Dolbeault Theorem A.6 to the differential graded holomorphic vector bundle $\left(\mathcal{D}_{\text {poly }}^{\prime}, d_{H}\right)$ of Definition A.1, whose local holomorphic sections are, by definition, local holomorphic differential operators on $X$, we may use the so-called Čech-Hochschild double complex

$$
\left(\check{C}\left(\mathfrak{U}, \mathcal{D}_{\text {poly }}\right), \check{\delta} \pm d_{H}\right),
$$

for a sufficiently nice open covering $\mathfrak{U}$ of $X$, in order to compute the Hochschild cohomology of $X$. Here, $\mathcal{D}_{\text {poly }}$ denotes the sheaf of holomorphic differential operators on $X$.

\section{A.3. Twisted presheaves of algebras.}

In order to give now a meaningful interpretation, in the framework of deformations of structures, of the second Hochschild cohomology group of a complex manifold $X$, we need a new sheaf-theoretical object. Let $X$ be a general topological space.

Definition A.7. A twisted presheaf $\mathcal{F}$ of algebras over $X$ (or, alternatively, an algebroid stack over $X$ ) consists of the following data:

i) an algebra $\mathcal{F}(U)$, for any open subset $U$ of $X$;

ii) a restriction homomorphism $\rho_{U, V}$ from $\mathcal{F}(U)$ to $\mathcal{F}(V)$;

iii) an invertible element $a_{U, V, W}$ of $\mathcal{F}(W)^{\times}$, for any three open subsets $W \subset V \subset U$ of $X$, satisfying the relations

$$
\begin{aligned}
\rho_{V, W} \circ \rho_{U, V} & =\operatorname{Ad}\left(a_{U, V, W}\right) \circ \rho_{U, W}, \quad W \subset V \subset U, \\
\rho_{U, Z}\left(a_{U, V, W}\right) a_{U, W, Z} & =a_{V, W, Z} a_{U, W, Z}, \quad Z \subset W \subset V \subset U,
\end{aligned}
$$

where Ad denotes the adjoint action of invertible elements of an algebra on itself by conjugation. 
Identity (A.3) is a coherence requirement for the restriction morphism $\rho_{U, V}$, in the following sense: for any four open subsets $Z \subset W \subset V \subset U$ of $X$, we have

$$
\begin{aligned}
\rho_{W, Z} \circ \rho_{V, W} \circ \rho_{U, V} & =\rho_{W, Z} \circ \operatorname{Ad}\left(a_{U, V, W}\right) \circ \rho_{U, W} \\
& =\operatorname{Ad}\left(\rho_{W, Z}\left(a_{U, V, W}\right)\right) \circ \rho_{W, Z} \circ \rho_{U, W} \\
& =\operatorname{Ad}\left(\rho_{W, Z}\left(a_{U, V, W}\right)\right) \circ \operatorname{Ad}\left(a_{U, W, Z}\right) \circ \rho_{U, Z} .
\end{aligned}
$$

On the other hand, we also have

$$
\begin{aligned}
\rho_{W, Z} \circ \rho_{V, W} \circ \rho_{U, V} & =\operatorname{Ad}\left(a_{V, W, Z}\right) \circ \rho_{V, Z} \circ \rho_{U, V} \\
& =\operatorname{Ad}\left(a_{V, W, Z}\right) \circ \operatorname{Ad}\left(a_{U, W, Z}\right) \circ \rho_{U, Z}
\end{aligned}
$$

A usual (pre)sheaf is a twisted presheaf of algebras, where we set $a_{U, V, W}=1$, for any three open subsets $W \subset V \subset U$. Further, if we assume the twisting elements $a_{U, V, W}$ to be central in the corresponding algebras, then the twisted presheaf of algebras $\mathcal{F}$ is a usual presheaf of algebras, endowed with central invertible elements $a_{U, V, W}$ satisfying the coherence relation (A.3).

Given two twisted presheaves of algebras $\mathcal{A}$ and $\mathcal{B}$ over the same topological space $X$, a morphism $\varphi$ from $\mathcal{A}$ to $\mathcal{B}$ consists of

i) an algebra morphism $\varphi_{U}$ from $\mathcal{A}(U)$ to $\mathcal{B}(U)$, for any open subset $U$ of $X$;

ii) an invertible element $c_{U, V}$ of $\mathcal{B}(V)^{\times}$, for any pair of open subsets $V \subset U$ of $X$, satisfying the relations

$$
\begin{aligned}
\varphi_{V} \circ \rho_{U, V}^{\mathcal{A}} & =\operatorname{Ad}\left(c_{U, V}\right) \circ \rho_{U, V}^{\mathcal{B}} \circ \varphi_{U}, \quad V \subset U, \\
\varphi_{W}\left(a_{U, V, W}\right) c_{U, W} & =c_{V, W} \rho_{V, W}^{\mathcal{B}}\left(c_{U, V}\right) b_{U, V, W}, \quad W \subset V \subset U,
\end{aligned}
$$

where $\rho_{U, V}^{\mathcal{A}}$, resp. $\rho_{U, V}^{\mathcal{B}}$, denotes the restriction morphism of the twisted sheaf of algebras $\mathcal{A}$, resp. $\mathcal{B} ; a_{U, V, W}$ and $b_{U, V, W}$ are the corresponding twisting elements.

An isomorphism from $\mathcal{A}$ to $\mathcal{B}$ is a morphism from $\mathcal{A}$ to $\mathcal{B}$, which admits a left- and rightinverse. Let $\mathcal{F}$ be a complex twisted presheaf over the topological space $X$.

Definition A.8. An order $n$ deformation of $\mathcal{F}$ is a twisted presheaf $\mathcal{A}$ of $k[\epsilon] / \epsilon^{n+1}$-algebras over $X$, such that $\mathcal{A} / \epsilon \mathcal{A} \cong \mathcal{F}$ as twisted presheaves of $k$-algebras.

In analogy with Gerstenhaber's interpretation of the second Hochschild cohomology group of an associative algebra $A$, we want to characterize the second Hochschild cohomology of the complex manifold $X$ in view of Definition A.8. Namely, we want to elucidate the fact that the second Hochschild cohomology group of $X$ parametrizes infinitesimal (i.e. order 1) deformations of the sheaf $\mathcal{O}_{X}$ of holomorphic functions on $X$ as a twisted presheaf of algebras, up to equivalence (an equivalence being, as usual, an isomorphism that reduces to the identity $\bmod \epsilon$ ).

For this purpose, we consider a general 2-cocycle in the Čech-Hochschild double complex of $X$ w.r.t. a sufficiently nice open covering $\mathfrak{U}$ of $X$ in the sense specified above. Such a 2-cocycle consists of three components $P^{(i, j)}$, where $i$ and $j$ are non negative integers such that $i+j=2$, and $P^{(i, j)} \in \check{C}^{j}\left(\mathfrak{U}, \mathcal{D}_{\text {poly }}^{i}\right)$.

Thus, $P^{(2,0)}$ is a Čech 0 -cochain with values in the sheaf $\mathcal{D}_{\text {poly }}^{2}$ of holomorphic bidifferential operators on $X, P^{(1,1)}$ is a Cech 1 -cocycle with values in the sheaf $\mathcal{D}_{\text {poly }}^{1}=\mathcal{D}_{\text {poly }}$ of holomorphic differential operators on $X$, and finally $P^{(0,2)}$ is a Cech 2-cocycle with values in the sheaf $\mathcal{O}_{X}$. The cochain condition $\left(\check{\delta} \pm d_{H}\right) P=0$ for the 2-cocycle $P$ is equivalent to the following set of identities (taking into account the Koszul sign convention for the 
Čech-Hochschild double complex):

$$
\begin{aligned}
d_{H} P^{(2,0)} & =0, \\
d_{H} P^{(1,1)}+\check{\delta} P^{(2,0)} & =0, \\
d_{H} P^{(0,2)}-\check{\delta} P^{(1,1)} & =0, \\
\check{\delta} P^{(0,2)} & =0 .
\end{aligned}
$$

The component $P^{(2,0)}$ consists of a family of holomorphic bidifferential operators on $X$ for any open subset $U_{\alpha}$ in $\mathfrak{U}$ : Identity (A.6) can be written more explicitly as

$$
f P_{\alpha}^{(2,0)}(g, h)+P_{\alpha}^{(2,0)}(f, g h)=P_{\alpha}^{(2,0)}(f g, h)+P_{\alpha}^{(2,0)}(f, g) h,
$$

for any triple of holomorphic functions $f, g, h$ on $U_{\alpha}$. We may thus consider the sheaf $\mathcal{A}_{\alpha}$ to be the restriction of the sheaf $\mathcal{O}_{X}[\epsilon] / \epsilon^{2}$ to $U_{\alpha}$, with deformed product given by

$$
(f, g)=\left(f_{0}+\epsilon f_{1}, g_{0}+\epsilon g_{1}\right) \mapsto f \star_{\alpha} g=f_{0} g_{0}+\epsilon\left(f_{0} g_{1}+f_{1} g_{0}+P_{\alpha}^{(2,0)}\left(f_{0}, g_{0}\right)\right):
$$

Identity (A.6) is easily verified to be equivalent to the fact that $\star_{\alpha}$ is an associative product modulo $\epsilon^{2}$; it is also obvious that the product $\star_{\alpha}$ reduces to the usual product modulo $\epsilon$. We notice that, for a deformed product $\star_{\alpha}$, for a choice of an open subset $U_{\alpha}$, we still want the unit 1 (the constant holomorphic function 1 ) to be a unit also w.r.t. $\star_{\alpha}$ : this is easily achieved by adding the condition that the holomorphic bidifferential operator $P_{\alpha}^{(2,0)}$ vanishes, whenever one of its arguments is a constant:

$$
\left(f_{0}+\epsilon f_{1}\right) \star_{\alpha} 1=1 \star_{\alpha}\left(f_{0}+\epsilon f_{1}\right)=f_{0}+\epsilon f_{1} \Longleftrightarrow P_{\alpha}^{(2,0)}\left(f_{0}, 1\right)=P_{\alpha}^{(2,0)}\left(1, f_{0}\right)=0 .
$$

Further, the component $P^{(1,1)}$ consists of a family of holomorphic differential operators $P_{\alpha \beta}^{(1,1)}$ on each (non-trivial) double intersection $U_{\alpha} \cap U_{\beta}$; additionally, Identity (A.7) can be rewritten as

$$
P_{\beta}^{(2,0)}(f, g)+f P_{\alpha \beta}^{(1,1)}(g)+g P_{\alpha \beta}^{(1,1)}(f)=P_{\alpha}^{(2,0)}(f, g)+P_{\alpha \beta}^{(1,1)}(f g),
$$

for any pair of holomorphic functions $f, g$ on $U_{\alpha} \cap U_{\beta}$. This means that the holomorphic differential operator $P_{\alpha \beta}^{(1,1)}$ defines an isomorphism

$$
\left(\left.\mathcal{A}_{\alpha}\right|_{U_{\alpha} \cap U_{\beta}, \star_{\alpha}}\right) \stackrel{\varphi_{\alpha \beta}}{\longrightarrow}\left(\left.\mathcal{A}_{\beta}\right|_{U_{\alpha} \cap U_{\beta}}, \star_{\beta}\right),
$$

where $\varphi_{\alpha \beta}$ is explicitly given by the formula

$$
f=f_{0}+\epsilon f_{1} \mapsto f_{0}+\epsilon\left(f_{1}+P_{\alpha \beta}^{(1,1)}\left(f_{0}\right)\right) .
$$

We want additionally the isomorphism $\varphi_{\alpha \beta}$ to preserve the unit of $\mathcal{A}_{\alpha}$ and $\mathcal{A}_{\beta}$, which turns out to be equivalent to the fact that the holomorphic differential operator $P_{\alpha \beta}^{(1,1)}$ vanishes on constant functions:

$$
\varphi_{\alpha \beta}(1)=1 \Leftrightarrow P_{\alpha \beta}^{(1,1)}(1)=0 .
$$

The third component $P^{(0,2)}$ is a family of holomorphic functions on each non-trivial triple intersection $U_{\alpha} \cap U_{\beta} \cap U_{\gamma}$ : since $\mathcal{O}_{X}$ is a sheaf of commutative algebras, we have $d_{H} P^{(0,2)}=0$. Hence, Identity (A.8) reduces to the simpler Čech cocycle condition

$$
P_{\alpha \beta}^{(1,1)}(f)+P_{\beta \gamma}^{(1,1)}(f)=P_{\alpha \gamma}^{(1,1)}(f),
$$

for any holomorphic function on $U_{\alpha} \cap U_{\beta} \cap U_{\gamma}$. This identity is obviously equivalent to the commutativity of the following diagram of sheaves (modulo $\epsilon$ and again by the commutativity 
of $\left.\mathcal{O}_{X}\right)$ :

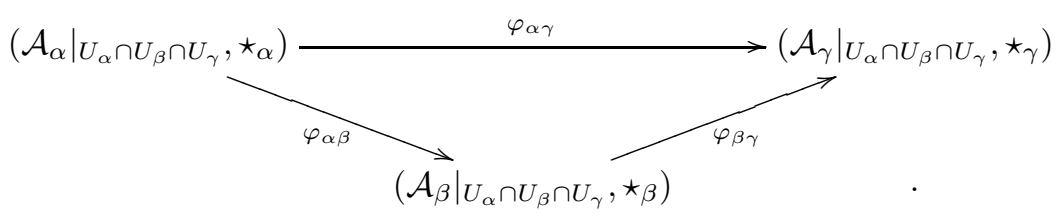

In spite of the observation made after Definition A.2, we may assume skew-symmetry w.r.t. the indices of all Čech cochains involved, whence

$$
P_{\alpha \alpha}^{(1,1)}=0
$$

which in turn implies $\varphi_{\alpha \alpha}=\mathrm{id}$.

Summing up what was done until here, we get, for each open subset $U_{\alpha}$, by means of $P_{\alpha}^{(2,0)}$, a sheaf $\left(\mathcal{A}_{\alpha}, \star_{\alpha}\right)$, which is obviously an infinitesimal deformation of the (trivial) twisted presheaf $\left.\mathcal{O}_{X}\right|_{U_{\alpha}}$; further, on each non-trivial intersection $U_{\alpha} \cap U_{\beta}, P_{\alpha \beta}^{(1,1)}$ determines an isomorphism between the sheaves $\left(\left.\mathcal{A}_{\alpha}\right|_{U_{\alpha} \cap U_{\beta}}, \star_{\alpha}\right)$ and $\left(\left.\mathcal{A}_{\beta}\right|_{U_{\alpha} \cap U_{\beta}}, \star_{\beta}\right)$, which, by (A.10), satisfies the cocycle condition. Hence, the sheaves $\left(\mathcal{A}_{\alpha}, \star_{\alpha}\right)$ define descent data, which can be glued together to give a sheaf $\mathcal{A}=\mathcal{A}(P)$, which is, by its very construction, an infinitesimal deformation of $\mathcal{O}_{X}$.

It remains to consider Identity (A.9), which can be written explicitly as

$$
P_{\beta \gamma \delta}^{(0,2)}+P_{\alpha \beta \delta}^{(0,2)}=P_{\alpha \gamma \delta}^{(0,2)}+P_{\alpha \beta \gamma}^{(0,2)}
$$

as a relation between functions on any non-trivial 4-fold intersection $U_{\alpha} \cap U_{\beta} \cap U_{\gamma} \cap U_{\delta}$. We observe first that, if $f$ is a holomorphic function on some open subset of $X$, which is contained in a non-trivial triple intersection $U_{\alpha} \cap U_{\beta} \cap U_{\gamma}$, then, setting

$$
a_{\alpha \beta \gamma}=1+\epsilon P_{\alpha \beta \gamma}^{(0,2)},
$$

we get an obviously invertible element of the algebras $\mathcal{A}_{\alpha}, \mathcal{A}_{\beta}$ and $\mathcal{A}_{\gamma}$ restricted on the triple intersection $U_{\alpha} \cap U_{\beta} \cap U_{\gamma}$, which is central in each of the three algebras w.r.t. the corresponding products:

$$
\left(f_{0}+\epsilon f_{1}\right) \star_{\alpha} a_{\alpha \beta \gamma}=a_{\alpha \beta \gamma} \star_{\alpha}\left(f_{0}+\epsilon f_{1}\right),
$$

and similar identities hold true, when $\star_{\alpha}$ is replaced by $\star_{\beta}$ or $\star_{\gamma}$ : this follows from the aforementioned fact that the holomorphic bidifferential operator $P_{\alpha}^{(2,0)}$ vanishes if one of its arguments is a constant. Furthermore, the central element $a_{\alpha \beta \gamma}$ is preserved by the isomorphisms $\varphi_{\alpha \beta}, \varphi_{\beta \gamma}$ and $\varphi_{\alpha \gamma}$, again as a consequence of the fact that the differential operators $P_{\alpha \beta}^{(1,1)}, P_{\beta \gamma}^{(1,1)}$ and $P_{\alpha \gamma}^{(1,1)}$ vanish, if their argument is a constant. Finally, the Cech cocycle relation can be reformulated as

$$
a_{\beta \gamma \delta} \star_{\alpha} a_{\alpha \beta \delta}=a_{\alpha \gamma \delta} \star_{\alpha} a_{\alpha \beta \gamma} ;
$$

we can also exchange the product $\star_{\alpha}$ by any other product $\star_{\beta}$, $\star_{\gamma}$ or $\star_{\delta}$, again the reason being that bidifferential operators vanish if one of their arguments is constant. Assuming furthermore that $P_{\alpha \beta \gamma}^{(0,2)}$ is skew-symmetric w.r.t. the indices, we have the additional relation

$$
a_{\alpha \beta \gamma}=1 \text {, }
$$

whenever two of the three indices are equal. Hence, the invertible elements $a_{\alpha \beta \gamma}$ define a twist in the sense of Definition A.7 on $\mathcal{A}$ : the triangle relation (A.2) for the restriction morphisms on $\mathcal{A}$ is trivially satisfied in spite of (A.11), while the coherence relation (A.3) holds true in spite of (A.12).

Thus, a 2-cocycle $P$ in Cech-Hochschild cohomology, which represents an element of the second Hochschild cohomology group of $X$, gives rise to an infinitesimal deformation $\mathcal{A}$ of $\mathcal{O}_{X}$ in the sense of Definition A.8. It remains to prove that two cohomologous 2-cocycles $P, Q$ in $\breve{C}$ ch-Hochschild cohomology give rise to isomorphic infinitesimal deformations $\mathcal{A}_{P}$, 
$\mathcal{A}_{Q}$ of $\mathcal{O}_{X}$. We assume therefore $P$ and $Q$ to be two cohomologous Cech-Hochschild, i.e. there is a Cech-Hochschild 1-cochain $R$, such that

$$
P-Q=\left(d_{H} \pm \check{\delta}\right) R
$$

which can be rewritten extensively as

$$
\begin{aligned}
& P^{(2,0)}-Q^{(2,0)}=d_{H} R^{(1,0)}, \\
& P^{(1,1)}-Q^{(1,1)}=d_{H} R^{(0,1)}-\check{\delta} R^{(1,0)}, \\
& P^{(0,2)}-Q^{(0,2)}=\check{\delta} R^{(0,1)} .
\end{aligned}
$$

The component $R^{(1,0)}$ consists of a family of holomorphic differential operators $R_{\alpha}^{(1,0)}$ on each element $U_{\alpha}$ of the chosen open covering. We define an isomorphism $\psi_{\alpha}$ via

$$
\left(\mathcal{A}_{\alpha}(P), \star_{\alpha}^{P}\right) \stackrel{\psi_{\alpha}}{\longrightarrow}\left(\mathcal{A}_{\alpha}(Q), \star_{\alpha}^{Q}\right),
$$

where the isomorphism $\psi_{\alpha}$ is explicitly defined as

$$
\psi_{\alpha}(f)=\psi_{\alpha}\left(f_{0}+\epsilon f_{1}\right)=f_{0}+\epsilon\left(f_{1}+R^{(1,0)}\left(f_{0}\right)\right) .
$$

Identity (A.13) can be rewritten explicitly as

$$
P_{\alpha}^{(2,0)}(f, g)+R^{(1,0)}(f g)=Q_{\alpha}^{(2,0)}(f, g)+f R^{(1,0)}(g)+g R^{(1,0)}(f),
$$

for any two holomorphic functions on $U_{\alpha}$ : the previous identity can be reformulated

$$
\psi_{\alpha}\left(\left(f_{0}+\epsilon f_{1}\right) \star_{\alpha}^{P}\left(g_{0}+\epsilon g_{1}\right)\right)=\psi_{\alpha}\left(f_{0}+\epsilon f_{1}\right) \star_{\alpha}^{Q} \psi_{\alpha}\left(g_{0}+\epsilon g_{1}\right),
$$

i.e. the isomorphism $\psi_{\alpha}$ is an algebra isomorphism, interchanging the deformed products $\star_{\alpha}^{P}$ and $\star_{\alpha}^{Q}$.

Further, Identity (A.14) can be rewritten in a simpler form, since the Hochschild differential of $R^{(0,1)}$ vanishes, due to the fact that $\mathcal{O}_{X}$ is a sheaf of commutative algebras, whence we get the simple relation

$$
P_{\alpha \beta}^{(1,1)}+R_{\beta}^{(1,0)}=Q_{\alpha \beta}^{(1,1)}+R_{\alpha}^{(1,0)}
$$

for holomorphic differential operators on any non-trivial double intersection $U_{\alpha} \cap U_{\beta}$. It is easy to check that the previous identity implies the commutativity of the following diagram:

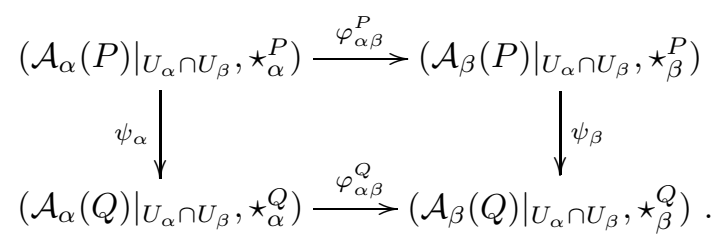

All these arguments imply that the local isomorphisms $\psi_{\alpha}$ can be glued together to define an isomorphism $\psi$ between the sheaves $\mathcal{A}(P)$ and $\mathcal{A}(Q)$, associated to the cocycles $P$ and $Q$ respectively by the above procedure.

Finally, we consider the holomorphic functions $R_{\alpha \beta}^{(0,1)}$ on any non-trivial double intersection $U_{\alpha} \cap U_{\beta}$. If we set

$$
c_{\alpha \beta}=1+\epsilon R_{\alpha \beta}^{(0,1)},
$$

we get elements of $\mathcal{A}_{\alpha}(Q)\left(U_{\alpha} \cap U_{\beta}\right)$ and of $\mathcal{A}_{\alpha}(Q)\left(U_{\alpha} \cap U_{\beta}\right)$. It is obvious that $c_{\alpha \beta}$ is an invertible element; since any holomorphic bidifferential operator $Q_{\alpha}^{(2,0)}$ vanishes, when one of its arguments is a constant, it follows that

$$
c_{\alpha \beta} \star_{\alpha}^{Q}\left(f_{0}+\epsilon f_{1}\right)=\left(f_{0}+\epsilon f_{1}\right) \star_{\alpha}^{Q} c_{\alpha \beta},
$$


and the same identity holds true replacing $\star_{\alpha}^{Q}$ by $\star_{\beta}^{Q}$. It is also easy to prove that the isomorphism $\psi_{\alpha}$ (as well as $\psi_{\beta}$ and $\psi_{\gamma}$ ) preserves the central invertible element $a_{\alpha \beta \gamma}^{P}$, i.e.

$$
\psi_{\alpha}\left(a_{\alpha \beta \gamma}\right)=a_{\alpha \beta \gamma},
$$

as a consequence of the fact that $\psi_{\alpha}$ preserves units w.r.t. the corresponding deformed products. The explicit form of Identity (A.15) is

$$
P_{\alpha \beta \gamma}^{(0,2)}+R_{\alpha \gamma}^{(0,1)}=Q_{\alpha \beta \gamma}^{(0,2)}+R_{\alpha \beta}^{(0,1)}+R_{\beta \gamma}^{(0,1)},
$$

which, using the centrality of $a_{\alpha \beta \gamma}^{P}, a_{\alpha \beta \gamma}^{Q}$ w.r.t. the deformed product $\star_{\alpha}^{Q}$ (as well as $\star_{\beta}^{Q}$ and $\left.\star_{\gamma}^{Q}\right)$, implies the relation

$$
\psi_{\alpha}\left(a_{\alpha \beta \gamma}^{P}\right) \star_{\alpha}^{Q} c_{\alpha \gamma}=c_{\beta \gamma} \star_{\alpha}^{Q} c_{\alpha \beta} \star_{\alpha}^{Q} a_{\alpha \beta \gamma}^{Q},
$$

and similarly when making corresponding changes of the deformed products involved, or of the isomorphisms $\psi_{\alpha}$. Hence, the elements $c_{\alpha \beta}$ define a twist $c$ for the morphism $\psi$, which satisfies (A.4) because of the centrality of $c_{\alpha \beta}$ w.r.t. the deformed products, and (A.5) by the previous identity. 


\section{REFERENCES}

[1] A. Alekseev and E. Meinrenken, The non-commutative Weil algebra, Invent. Math. 139 (2000), 135-172.

[2] A. Alekseev and E. Meinrenken, Lie theory and the Chern-Weil homomorphism, Ann. Sci. École Norm. Sup. 38 (2005), no. 2, 303-338.

[3] D. Arnal, D. Manchon, and M. Masmoudi, Choix des signes pour la formalité de M. Kontsevich, Pacific J. Math. 203 (2002), no. 1, 23-66.

[4] D. Bar-Natan, T. Le, and D. Thurston, Two applications of elementary knot theory to Lie algebras and Vassiliev invariants, Geom. Topol. 7 (2003), 1-31.

[5] D. Calaque, V. Dolgushev and G. Halbout, Formality theorems for Hochschild chains in the Lie algebroid setting, J. Reine Angew. Math. 612 (2007), 81-127

[6] D. Calaque and M. Van den Bergh, Hochschild cohomology and Atiyah classes, preprint arXiv:math/0708.2725.

[7] A. Căldăraru, The Mukai pairing, II: the Hochschild-Kostant-Rosenberg isomorphism, Adv. Math. 194 (2005), no. 1, 34-66.

[8] A. Cattaneo and G. Felder, Relative formality theorem and quantisation of coisotropic submanifolds, Adv. Math. 208 (2007), no. 2, 521-548.

[9] A. Cattaneo, B. Keller, C. Torossian, and A. Bruguières, Déformation, Quantification, Théorie de Lie, Panoramas et Synthèses 20 (2005), SMF.

[10] C. Chevalley and S. Eilenberg, Cohomology theory of Lie groups and Lie algebras, Trans. Amer. Math. Soc. 63 (1948), 85-124.

[11] V. Dolgushev, Covariant and equivariant formality theorem, Adv. Math 191 (2005), no. 1, 51-101.

[12] M. Duflo, Caractères des groupes et des algèbres de Lie résolubles, Ann. Sci. École Norm. Sup. 3 (1970), 23-74.

[13] M. Duo, Opérateurs différentiels bi-invariants sur un groupe de Lie, Ann. Sci. École Norm. Sup. 10 (1977), 265-288.

[14] B. Fedosov, A simple geometrical construction of deformation quantization, J. Diff. Geom. 40 (1994), 213-238.

[15] W. Fulton and R. Mac Pherson, A compactification of configuration spaces, Ann. of Math. (2) 139 (1994), no. 1, 183-225.

[16] M. Gerstenhaber, On the deformation of rings and algebras, Ann. Math. 79 (1964), no. 1, 59-103.

[17] P. Griffith and J. Harris, Principles of algebraic geometry, Wiley Classics Library, 1994.

[18] Harish-Chandra, On some applications of the universal enveloping algebra of a semisimple Lie group, Trans. Amer. Math. Soc. 70 (1951), 28-96.

[19] G. Hochschild, B. Kostant, and A. Rosenberg, Differential forms on regular affine algebras, Trans. Amer. Math. Soc. 102 (1962), 383-408.

[20] A. Khovanskii, On a lemma of Kontsevich, Funct. An. Appl. 31 (1997), no. 4, 296-298.

[21] A. Kirillov, Elements of the Theory of Representations, Springer-Verlag 1975.

[22] M. Kontsevich, Deformation quantization of Poisson manifolds, Lett. Math. Phys. 66 (2003), no. 3, $157-216$.

[23] A. Kricker, Non-commutative Chern-Weil theory and the combinatorics of wheeling, preprint arXiv:math/0612653.

[24] J. Leray, L'anneau d'homologie d'une représentation, C.R.A.S. 222 (1946), 1366-1368.

[25] J. Leray, Structure de l'anneau d'homologie d'une représentation, C.R.A.S. 222 (1946), 1419-1422.

[26] D. Manchon and C. Torossian, Cohomologie tangente et cup-produit pour la quantification de Kontsevich, Ann. Math. Blaise Pascal 10 (2003), no. 1, 75-106.

[27] M. Pevzner and C. Torossian, Isomorphisme de Duflo et cohomologie tangentielle, J. Geom. Phys. 51 (2004), no. 4, 486-505.

[28] L. Politsel'skii, Nonhomogeneous quadratic duality and curvature, Funkts. An. Pril. 27 (1993), no. 3, $57-66$.

[29] B. Shoikhet, On the Duflo formula for $L_{\infty}$-algebras and Q-manifolds, preprint arXiv:math.QA/9812009.

[30] B. Shoikhet, Kontsevich formality and $P B W$ algebras, preprint arXiv:math/0708.1634.

[31] J. Stasheff, Homotopy associativity of H-spaces I \& II, Trans. AMS 108 (1963), 275-312.

[32] M. Van den Bergh, The Kontsevich weight of a wheel with spokes pointing outward, to appear in the special issue of Alg. Repr. Theory in honor of F. van Oystaeyen (preprint arXiv.math/0710.2411).

[33] J. Vey, Déformation du crochet de Poisson sur une variété symplectique, Comm. Math. Helv. 50 (1975), 421-454.

[34] T. Willwacher, A counterexample to the quantizability of modules, Lett. Math. Phys. 81 (2007), no. 3, 265-280.

E-mail address: calaque@math.univ-lyon1.fr, damien.calaque@math.ethz.ch

E-mail address: carlo.rossi@math.ethz.ch 